\title{
Mapping photodissociation and shocks in the vicinity of Sagittarius $A^{* \star}, \star \star$
}

\author{
M. A. Amo-Baladrón ${ }^{1}$, J. Martín-Pintado ${ }^{1}$, and S. Martín 2,3 \\ ${ }^{1}$ Centro de Astrobiología (CSIC/INTA), Ctra. de Torrejón a Ajalvir km 4, 28850 Torrejón de Ardoz, Madrid, Spain \\ e-mail: arancha@damir.iem.csic.es \\ 2 European Southern Observatory, Alonso de Córdova 3107, Vitacura, Casilla 19001, Santiago 19, Chile \\ ${ }^{3}$ Harvard-Smithsonian Center for Astrophysics, 60 Garden Street, Cambridge, MA 02138, USA
}

Received 26 October 2009 / Accepted 12 October 2010

\begin{abstract}
Aims. We study the chemistry in the harsh environments of galactic nuclei using the nearest one, the Galactic center (GC). Methods. We have obtained maps of the molecular emission within the central five arcminutes (12 pc) of the GC in selected molecular tracers: $\mathrm{SiO}(2-1), \mathrm{HNCO}\left(5_{0,5}-4_{0,4}\right)$, and the $J=1 \rightarrow 0$ transition of $\mathrm{H}^{13} \mathrm{CO}^{+}, \mathrm{HN}^{13} \mathrm{C}$, and $\mathrm{C}^{18} \mathrm{O}$ at an angular resolution of $30^{\prime \prime}$ $(1.2 \mathrm{pc})$. The mapped region includes the circumnuclear disk (CND) and the two surrounding giant molecular clouds (GMCs) of the Sgr A complex, known as the 20 and $50 \mathrm{~km} \mathrm{~s}^{-1}$ molecular clouds. Additionally, we simultaneously observed the $J=2 \rightarrow 1$ and $J=3 \rightarrow 2$ transitions of $\mathrm{SiO}$ toward selected positions to estimate the physical conditions of the molecular gas using the large velocity gradient approximation.

Results. The $\mathrm{SiO}(2-1)$ emission shows all the molecular features identified in previous studies, covering the same velocity range as the $\mathrm{H}^{13} \mathrm{CO}^{+}(1-0)$ emission, which also presents a similar distribution. In contrast, $\mathrm{HNCO}(5-4)$ emission appears in a narrow velocity range mostly concentrated in the 20 and $50 \mathrm{~km} \mathrm{~s}^{-1}$ GMCs. A similar trend follows the $\mathrm{HN}^{13} \mathrm{C}(1-0)$ emission. The HNCO column densities and fractional abundances present the highest contrast, with difference factors of $\geq 60$ and 28 , respectively. Their highest values are found toward the cores of the GMCs, whereas the lowest ones are measured at the CND. SiO abundances do not follow this trend, with high values found toward the CND, as well as the GMCs. By comparing our abundances with those of prototypical Galactic sources we conclude that $\mathrm{HNCO}$, similar to $\mathrm{SiO}$, is ejected from grain mantles into gas-phase by nondissociative $\mathrm{C}$-shocks. This results in the high abundances measured toward the CND and the GMCs. However, the strong UV radiation from the Central cluster utterly photodissociates $\mathrm{HNCO}$ as we get closer to the center, whereas $\mathrm{SiO}$ seems to be more resistant against UV-photons or it is produced more efficiently by the strong shocks in the CND. This UV field could be also responsible for the trend found in the $\mathrm{HN}^{13} \mathrm{C}$ abundance.

Conclusions. We discuss the possible connections between the molecular gas at the CND and the GMCs using the HNCO/SiO, $\mathrm{SiO} / \mathrm{CS}$, and $\mathrm{HNCO} / \mathrm{CS}$ intensity ratios as probes of distance to the Central cluster. In particular, the $\mathrm{HNCO} / \mathrm{SiO}$ intensity ratio is proved to be an excellent tool for evaluating the distance to the center of the different gas components.
\end{abstract}

Key words. astrochemistry - ISM: clouds - ISM: molecules - Galaxy: abundances - Galaxy: nucleus - Galaxy: center

\section{Introduction}

Its proximity ( $8.0 \pm 0.5 \mathrm{kpc}$; Reid 1993) makes the Galactic center (GC) the best laboratory for understanding the heating and chemistry of the interstellar medium (ISM) in the harsh environment of galaxy nuclei. Just in its central $5 \operatorname{arcmin}(12 \mathrm{pc})$, one can find a massive black hole surrounded by a rotating circumnuclear disk (CND) of dust and gas, H II regions, massive stellar clusters, two supernova remants (SNRs), and two giant molecular clouds (GMCs). All these features coexist, interact, and can be spatially resolved at a resolution currently impossible even in the nearest extragalactic nucleus.

At its dynamical center, the GC harbors a massive black hole of $\sim 4.0 \times 10^{6} M_{\odot}$ (Ghez et al. 2005) that is associated with the strong compact source of nonthermal emission

\footnotetext{
* Appendices are only available in electronic form at http: //www . aanda.org

$\star \star$ Based on observations with the IRAM 30-m telescope. IRAM is supported by CNRS/INSU (France), the MPG (Germany) and the IGN (Spain).
}

Sgr A*, visible in radio continuum maps (Yusef-Zadeh \& Morris 1987). Converging to $\operatorname{Sgr} \mathrm{A}^{*}$, there are arc-shaped ionized gas streamers with a minispiral morphology (Sgr A West) that seem to be feeding the nucleus (Lo \& Claussen 1983; Roberts \& Goss 1993). Both the black hole and Sgr A West are surrounded by a ring of molecular gas and dust (the CND; Becklin et al. 1982; Güsten et al. 1987), whose inner edge suffers UV-photoionization (Roberts \& Goss 1993) due to a dense stellar population of massive stars in the Central cluster (Krabbe et al. 1995; Figer 2008).

The CND has been observed from radio to infrared wavelengths in molecular gas tracers, continuum, and atomic species (see Table 1 for references). These studies have characterized the CND as dense $\left(\sim 10^{5-6} \mathrm{~cm}^{-3}\right.$; Marr et al. 1993; Marshall et al. 1995 ), clumpy, and very turbulent (with large linewidths). It extends from its inner edge, $1.6 \mathrm{pc}$ from Sgr $\mathrm{A}^{*}$, to $\gtrsim 2 \mathrm{pc}$, according to interferometric studies (Wright et al. 2001; Christopher et al. 2005), and it has an inclination (rotation axis from the line of sight; LOS) of about $60^{\circ}-70^{\circ}$, with the major axis aligned approximately along the Galactic plane (position angle of $\sim 30^{\circ}$ east of north; Güsten et al. 1987; Jackson et al. 1993; 
Table 1. Observations of the CND.

\begin{tabular}{|c|c|}
\hline Tracers & References \\
\hline \multirow{2}{*}{$\mathrm{H}_{2}$} & Gatley et al. (1986) \\
\hline & Yusef-Zadeh et al. (2001) \\
\hline \multirow[t]{3}{*}{$\mathrm{CO}(1-0)$} & Liszt et al. (1985) \\
\hline & Serabyn et al. (1986) \\
\hline & Liszt \& Burton (1995) \\
\hline${ }^{13} \mathrm{CO}$ and $\mathrm{C}^{18} \mathrm{O}(2-1)$ & Zylka et al. (1990) \\
\hline \multirow[t]{3}{*}{$\mathrm{CO}(3-2)$} & Sutton et al. (1990) \\
\hline & Dent et al. (1993) \\
\hline & Liszt \& Burton (1995) \\
\hline $\mathrm{CO}(7-6)$ & Bradford et al. (2005) \\
\hline $\operatorname{CS}(2-1)$ & Serabyn et al. (1986) \\
\hline$C^{34} S(2-1)$ & Zylka et al. (1990) \\
\hline $\operatorname{CS}(7-6)$ & Montero-Castaño et al. (2009) \\
\hline \multirow[t]{4}{*}{$\mathrm{HCN}(1-0)$} & Güsten et al. (1987) \\
\hline & Marr et al. (1993) \\
\hline & Wright et al. (2001) \\
\hline & Christopher et al. (2005) \\
\hline \multirow[t]{2}{*}{$\mathrm{HCN}(3-2)$} & Jackson et al. (1993) \\
\hline & Marshall et al. (1995) \\
\hline \multirow[t]{2}{*}{$\mathrm{HCN}(4-3)$} & Marshall et al. (1995) \\
\hline & Montero-Castaño et al. (2009) \\
\hline $\mathrm{H}^{13} \mathrm{CN}(1-0)$ & Marr et al. (1993) \\
\hline \multirow[t]{4}{*}{$\mathrm{HCO}^{+}(1-0)$} & Marr et al. (1993) \\
\hline & Wright et al. (2001) \\
\hline & Christopher et al. (2005) \\
\hline & Shukla et al. (2004) \\
\hline $\mathrm{NH}_{3}(1,1)(2,2)$ & Coil \& Ho $(1999,2000)$ \\
\hline $\mathrm{NH}_{3}(1,1)(2,2)(3,3)$ & McGary et al. (2001) \\
\hline $\mathrm{NH}_{3}(6,6)$ & Herrnstein \& Ho (2002) \\
\hline \multirow{5}{*}{$\begin{array}{l}\text { Continuum at } \\
\mathrm{mm} \text { and sub-mm } \\
\text { wavelengths }\end{array}$} & Mezger et al. (1989) \\
\hline & Zylka et al. (1990) \\
\hline & Davidson et al. (1992) \\
\hline & Dent et al. (1993) \\
\hline & Telesco et al. (1996) \\
\hline \multirow{5}{*}{$\begin{array}{l}\text { Atomic species } \\
(\mathrm{HI},[\mathrm{O} I],[\mathrm{C}],[\mathrm{C} \mathrm{II}])\end{array}$} & Liszt et al. (1985) \\
\hline & Genzel et al. (1985) \\
\hline & Lugten et al. (1986) \\
\hline & Jackson et al. (1993) \\
\hline & Serabyn et al. (1994) \\
\hline
\end{tabular}

Marshall et al. 1995). The gas is moving in circular orbits, rotating around the nucleus with a velocity of $110 \mathrm{~km} \mathrm{~s}^{-1}$ (Liszt et al. 1985; Marr et al. 1993), but it also presents noncircular motions (e.g.: Güsten et al. 1987; Jackson et al. 1993; Marshall et al. 1995; Shukla et al. 2004; Christopher et al. 2005). The CND does not appear to be an equilibrium structure. It seems to consist of several separated streamers in rotation around the nucleus (Jackson et al. 1993; Wright et al. 2001). Virial analysis suggests that the CND could be composed of high-density clumps $\left(\sim 10^{6-7} \mathrm{~cm}^{-3}\right)$ that could withstand the tidal shear making the CND a long-lived structure (Jackson et al. 1993; Shukla et al. 2004; Christopher et al. 2005; Montero-Castaño et al. 2009). However, Güsten et al. (1987) derived significantly lower densities $\left(\sim 10^{5} \mathrm{~cm}^{-3}\right)$ from their interferometric mapping of $\mathrm{HCN}$, which are consistent with the densities estimated from warm atomic gas (Genzel et al. 1985) and CO multitransitional observations (Harris et al. 1985).

One question that remains without a conclusive answer is how the CND is fed. Since this structure is not just a transient feature losing material through infall (probably via Sgr A West, Montero-Castaño et al. 2009), there must be gas feeding the disk. Two GMCs that lie near the GC are the best candidates to feed the CND: the $20 \mathrm{~km} \mathrm{~s}^{-1}$ and the $50 \mathrm{~km} \mathrm{~s}^{-1}$ GMCs (M-0.13-0.08 and M-0.02-0.07, respectively; Güsten et al. 1981; Güsten \& Henkel 1983). Both are connected by a ridge of compressed dust and gas that warps around the SNR Sgr A East. This SNR lies behind or contains Sgr A West and appears as an expanding shell of synchrotron emission in radio continuum maps (Yusef-Zadeh \& Morris 1987; Pedlar et al. 1989). But it is not the only SNR in the region. G 359.92-0.09, located to the south of Sgr A East (centered at $\Delta \alpha=120^{\prime \prime}, \Delta \delta=-180^{\prime \prime}$ with respect to Sgr A*), is expanding into Sgr A East producing its concave southeastern edge (Coil \& Ho 2000). Both SNRs seem to interact with the surrounding material pushing it away from or toward the nucleus. As a result, several streamers of gas and dust that could be feeding the CND have been formed (Lee et al. 2008).

Several studies at different wavelengths have attempted to establish the relation between all the components in the Sgr A complex and their 3-dimensional (3D) location (see Coil \& Ho 2000; Herrnstein \& Ho 2005; Lee et al. 2008, and references therein). For the sake of clarity, we show in Fig. 1 a 2D sketch of the region with all the previous identified components, following the notation of Herrnstein \& Ho (2005) for the molecular features in most of the cases. These studies have resulted in the following picture:

1. The HII region Sgr A West, also known as the Minispiral, is located in front of or inside the SNR Sgr A East (Yusef-Zadeh \& Morris 1987; Pedlar et al. 1989). Moreover, Maeda et al. (2002) place Sgr A*, Sgr A West, and the CND (hereafter the nuclear region) just inside the leading edge of Sgr A East.

2. The SNR Sgr A East is pushing the $50 \mathrm{~km} \mathrm{~s}^{-1}$ GMC both to the east and behind the SNR along the LOS (Coil \& Ho 2000; Park et al. 2004).

3. The $20 \mathrm{~km} \mathrm{~s}^{-1} \mathrm{GMC}$ is located in front of the nucleus along the LOS (Güsten \& Downes 1980; Park et al. 2004) and Sgr A East could also be expanding into this GMC (Mezger et al. 1989).

4. Both GMCs are connected by the Molecular Ridge, whose northern part is farther away from the Sun along the LOS than the southern part (Coil \& Ho 2000). Lee et al. (2008) claim that the SNR Sgr A East could interact with the northern part of the ridge nearly perpendicular to the LOS, locating the northern part of the ridge slightly behind this SNR.

5. The SNR G 359.92-0.09 is interacting with the southern part of the Molecular Ridge, the eastern edge of the $20 \mathrm{~km} \mathrm{~s}^{-1} \mathrm{GMC}$, and the southern edge of Sgr A East (Coil \& Ho 2000).

6. The Western Streamer, a ridge of emission seen in $\mathrm{NH}_{3}$ bordering the western edge of Sgr A East, is highly inclined with respect to the LOS, and it is expanding outward as does this SNR (McGary et al. 2001; Lee et al. 2008).

7. The CND could be connected with the GMCs through three molecular gas streamers: the Southern Streamer (Okumura et al. 1991), the Northern Ridge (McGary et al. 2001), and the $50 \mathrm{~km} \mathrm{~s}^{-1}$ Streamer (Szczepanski et al. 1991).

8. Finally, high-velocity $\mathrm{C}^{18} \mathrm{O}(2-1)$ emission between -80 and $20 \mathrm{~km} \mathrm{~s}^{-1}$ located in projection close to the nuclear region have been identified by Genzel et al. (1990). In our maps, this emission appears as a small blue-shifted molecular cloud that we will refer it to as Cloud A.

The main heating mechanism in this complex region (CND and its central cavity) seems to be a combination of photoelectric heating by UV photons and mechanical heating by dissipation of supersonic turbulence in shocks (Bradford et al. 2005; Montero-Castaño et al. 2009). Theoretical models for the 


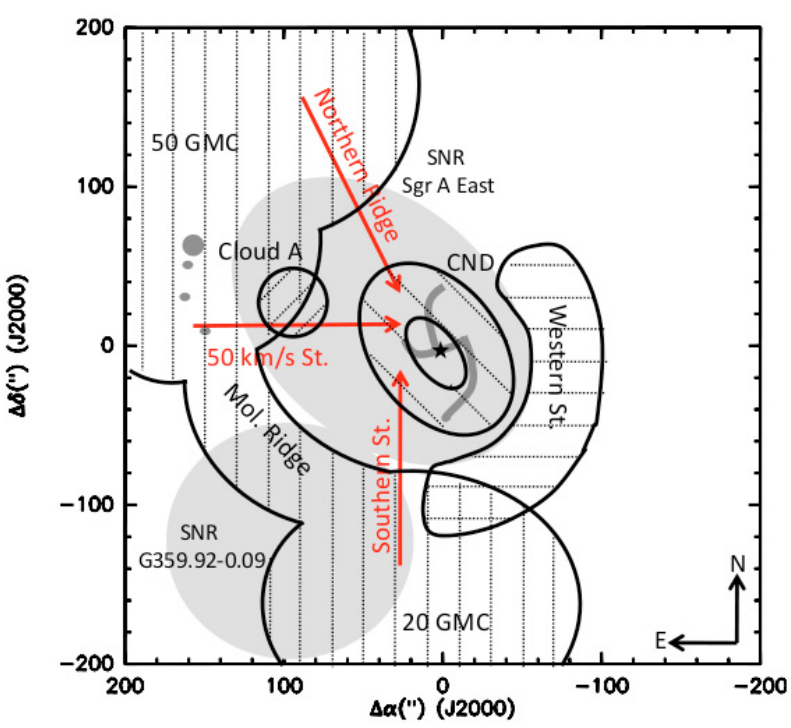

Fig. 1. Sketch of the 12 central parsecs of the Galaxy. Gray regions represents radio continuum features. Big ellipses correspond to the SNRs Sgr A East and G 359.92-0.09, the central minispiral to the H II region Sgr A West, and the four little circles to the Sgr A East compact H II regions. The central star represents Sgr A*. Molecular gas features are represented by stripped regions, whereas red arrows toward the center point out the possible connections between the CND (the elliptic disk) and the CMCs of this region. See the text for more details.

formation of the CND and the Northern Arm of the ionized Minispiral invoke infalling clouds as a result of cloud-cloud collisions and predict frequent collisions between clumps that would produce shock heating (Sanders 1998; Vollmer \& Duschl 2002). Direct probes of shocks in the CND are the detections of $1720 \mathrm{MHz}$ OH masers with extreme velocities $\left( \pm 130 \mathrm{~km} \mathrm{~s}^{-1}\right)$ at its lobes reported by Sjouwerman \& Pihlström (2008). These authors claim that this type of maser is collisionally pumped and originates in postshock regions. Bradford et al. (2005), modeling CO rotational lines, reached the conclusion that UV heating alone is not able to match the $\mathrm{CO}$ luminosities, and therefore mechanical energy dissipated into the gas in low-velocity (10-20 $\left.\mathrm{km} \mathrm{s}^{-1}\right) \mathrm{C}$-shocks with magnetic fields of $0.3-0.5 \mathrm{mG}$ could be the primary source of energy for the bulk of the heating of the CND material. Additional probes of shocks and/or UV radiation come from prominent $\mathrm{H}_{2}$ emission found in the CND, in its southwest and northeast lobes, just where $\mathrm{HCN}(1-0)$ emission is stronger (Christopher et al. 2005). Moreover, there could be another source of shocks in the CND. In their study of the possible interactions between Sgr A East and the molecular gas features of the nuclear region, Lee et al. (2008) have found that the southern part of the CND and its northwestern region could lie in front of the SNR Sgr A East and could be pushed toward us. In fact, several $1720 \mathrm{MHz} \mathrm{OH}$ masers have been detected tracing the interaction of this SNR with the surrounding GMCs (Sjouwerman \& Pihlström 2008).

Therefore, the energetics of the central region seem to be dominated by UV radiation and magnetohydrodynamical shocks. Chemical complexity can potentially be used to locate the different molecular components along the LOS in the central region by measuring the effects of photodissociation produced by the UV radiation from the Central cluster. It has been proposed that $\mathrm{SiO}, \mathrm{HNCO}$, and $\mathrm{CS}$ are among the best molecular tracers of the different ISM heating mechanisms. All of them have high critical densities suitable for
GC physical conditions, so they trace similar gas components, but each species is sensitive to different heating mechanisms through their particular chemistry. $\mathrm{SiO}$ is a well-known shock tracer (Martín-Pintado et al. 1992), HNCO seems to be also associated to grain chemistry but it is strongly affected by photodissociation (Martín et al. 2008, 2009a), whereas CS is marginally enhanced in UV (Goicoechea et al. 2006; Martín et al. 2008) and shocked environments (Requena-Torres et al. 2006). The $\mathrm{SiO} / \mathrm{CS}$ and $\mathrm{HNCO} / \mathrm{CS}$ ratios can then be used to trace shocks in well-shielded regions by the enhancement of the $\mathrm{SiO}$ and $\mathrm{HNCO}$ abundances with respect to that of CS. In unshielded regions illuminated by UV radiation and with strong shocks, one expects to measure an enhancement of the $\mathrm{SiO} / \mathrm{CS}$ ratio and the decrease in the $\mathrm{HNCO} / \mathrm{CS}$ ratio.

In this paper we present spectral imaging of the molecular emission from the central region of the GC (12 pc), which includes the CND and two GMCs of the Sgr A complex. The selected molecular lines were the $J=2 \rightarrow 1$ transition of $\mathrm{SiO}$, the $J=5_{0,5} \rightarrow 4_{0,4}$ transition of $\mathrm{HNCO}$ (hereafter $\mathrm{HNCO}(5-4)$ ), and the $J=1 \rightarrow 0$ transition of $\mathrm{H}^{13} \mathrm{CO}^{+}, \mathrm{HN}^{13} \mathrm{C}$, and ${ }^{18} \mathrm{CO}$. In Sect. 2 the observation and data reduction procedures are described. Section 3 presents the data for each observed species in selected velocity ranges for better comparison with radio continuum maps. Kinematics is described with declination-velocity maps at constant right ascension intervals. Physical conditions at selected positions, as well as column densities and fractional abundances, are derived in Sects. 4 and 5. Finally, in Sect. 6 the changes found in molecular abundances are discussed, compared to prototypical sources, and used to probe the different heating mechanisms in the region and to establish the location of the different molecular components in the nuclear region relative to the Central cluster.

\section{Observations}

The observations were carried out with the IRAM-30 m radiotelescope at Pico Veleta (Spain) during the summer of 2006. The mapping of the lines were made simultaneously by tuning the two $3 \mathrm{~mm}$ receivers (A and $\mathrm{B}$ ) to two different frequencies: 86.892 and $109.905 \mathrm{GHz}$. Within the first frequency band we observed the $\mathrm{SiO}(2-1), \mathrm{H}^{13} \mathrm{CO}^{+}(1-0)$, and $\mathrm{HN}^{13} \mathrm{C}(1-0)$ lines, whereas within the second frequency band we observed the $\mathrm{HNCO}(5-4)$ and $\mathrm{C}^{18} \mathrm{O}(1-0)$ lines. Both receivers were tuned to a single sideband with image rejections of $30 \mathrm{~dB}$ and $25 \mathrm{~dB}$. The system temperatures ranged between 154-176 $\mathrm{K}$ and 222-265 K during the observations for the lower and higher frequency setups, respectively. We used filterbanks $(512 \times 1 \mathrm{MHz})$ as spectrometers, providing a spectral resolution of $3.4 \mathrm{~km} \mathrm{~s}^{-1}$ at $86.9 \mathrm{GHz}$, and $2.7 \mathrm{~km} \mathrm{~s}^{-1}$ at $109.9 \mathrm{GHz}$, enough to resolve the wide emission lines of the $\mathrm{GC}\left(\sim 20 \mathrm{~km} \mathrm{~s}^{-1}\right)$.

Data were taken using the on-the-fly (OTF) technique with a telescope dump time (integration time on each position) of $1 \mathrm{~s}$ and was fully sampled at $86.9 \mathrm{GHz}$. The maps cover an area of $300^{\prime \prime} \times 300^{\prime \prime}(12 \times 12 \mathrm{pc}$ at the GC distance of $8.0 \mathrm{kpc})$ centered on the position of the radio source $\mathrm{Sgr}^{*}$ $\left((\alpha, \delta)_{\mathrm{J} 2000}=17^{\mathrm{d}} 45^{\mathrm{m}} 40^{\mathrm{s}} .031,-29^{\circ} 00^{\prime} 28^{\prime \prime} .58\right)$. We made eight coverages of the region alternating the scanning direction between right ascension and declination. The orthogonal covers were made in such a way that the $\sim 200^{\prime \prime} \times 200^{\prime \prime}$ central region was finally mapped in both directions, in order to minimize possible scanning artifacts. The half-power beamwidth $(H P B W)$ of the telescope was $28^{\prime \prime}$ at the frequency of $86.9 \mathrm{GHz}$, and $22^{\prime \prime}$ at $109.9 \mathrm{GHz}$. 
Spectra were calibrated using the standard dual load system. We used the antenna temperature scale $\left(T_{\mathrm{A}}^{*}\right)$ for the line intensities because the emission is rather extended and fills the beam. We used $(l, b)=\left(-0.02^{\circ},-0.20^{\circ}\right)$ as reference position, close enough to minimize the time lost due to telescope switching and differences in atmospheric airmass. This position shows almost no emission in the CS(1-0) survey of Tsuboi et al. (1999). To confirm that the reference was free of emission, it was checked against another further reference $\left((l, b)=\left(-0.25^{\circ},-0.25^{\circ}\right)\right)$. The reference position shows no emission at a noise level of 12 and $23 \mathrm{mK}$ for the low and high frequency spectra, respectively. A focus check was performed at the beginning of each observing session, and pointing was checked at the beginning and in the middle of each observing session.

Data reduction was done with the GILDAS package. The reduction process was as follows. First, bad channels and standing waves were removed from the spectra, and typical baselines up to order 3 , in the case of spectra at $86.9 \mathrm{GHz}$, and up to order 5 , at $109.9 \mathrm{GHz}$, were subtracted. In the case of the highfrequency spectra, a baseline of order 0 would had been enough, but a baseline of higher degree was needed to correct the baseline at the backend edges. We also used the CS data cube of the $J=1 \rightarrow 0$ line taken by Tsuboi et al. (1999). To make all our molecular line cubes and that of CS(1-0) comparable, our spectra were resampled to the same velocity resolution as for the CS(1-0) cube $\left(5 \mathrm{~km} \mathrm{~s}^{-1}\right)$. Finally, we constructed all the molecular line maps with an $H P B W$ of $30^{\prime \prime}$ (the beam of the $86.9 \mathrm{GHz}$ and $\mathrm{CS}(1-0)$ cubes). The final data cubes have an rms noise per channel of 27,37 , and $100 \mathrm{mK}$ for the $\mathrm{SiO} / \mathrm{H}^{13} \mathrm{CO}^{+} / \mathrm{HN}^{13} \mathrm{C}$, $\mathrm{HNCO} / \mathrm{C}^{18} \mathrm{O}$, and $\mathrm{CS}$ lines, respectively.

We also present observations of the $J=2 \rightarrow 1$ and $J=3 \rightarrow 2$ transitions of $\mathrm{SiO}$ taken simultaneously at selected positions. The $H P B W$ of the telescope at the rest frequency of the $\mathrm{SiO}(3-2)$ transition $(130.26861 \mathrm{GHz})$ was $19^{\prime \prime}$. The system temperature was around 150 for the (2-1) and $270 \mathrm{~K}$ for (3-2) transitions, resulting in an rms noise $\leq 32 \mathrm{mK}$. The $\left(40^{\prime \prime},-120^{\prime \prime}\right)$ position was observed in a past campaign and presents system temperatures of 278 and $423 \mathrm{~K}$, and rms noise values of 56 and $80 \mathrm{mK}$ for the (2-1) and (3-2) transitions.

\section{Results}

In this section we analyze the morphology and kinematics of the emission for every observed molecular species. Figure 2 shows the integrated intensity maps of the observed molecular lines in 9 velocity intervals, from -125 to $130 \mathrm{~km} \mathrm{~s}^{-1}$, selected to highlight the different components seen in the data cubes (velocitychannel maps are available in Figs. A.1-A.5). In this figure, we also show the CS(1-0) integrated intensity maps of Tsuboi et al. (1999) for comparison. The rms noise for the integrated-velocity maps $\left(\sigma_{\Delta v}\right)$ has been calculated from the rms noise of the original data cubes $\left(\sigma_{\mathrm{ch}}\right.$; Sect. 2$)$ using the formula

$\sigma_{\Delta v}=\frac{\sigma_{\mathrm{ch}}}{\sqrt{N_{\mathrm{ch}}}} \cdot \Delta v$

where $N_{\text {ch }}$ is the number of integrated velocity channels and $\Delta v$ the velocity range where the emission is integrated.

Figure 3 shows declination-velocity maps at constant right ascension. To increase the signal-to-noise ratio and to clearly show the gas kinematics of the components associated with the most outstanding features, we have also averaged the declination-velocity cuts at four selected right ascension intervals.
Finally, in Fig. 4 we compare the integrated $\mathrm{SiO}(2-1)$ emission maps with the radio continuum image at $6 \mathrm{~cm}$ (Yusef-Zadeh \& Morris 1987), where we can distinguish the thermal emission from Sgr A West (the gray minispiral in Fig. 1). This feature is composed of three ionized gas streamers: the Western Arc (the southwestern photoionized inner edge of the CND; Serabyn \& Lacy 1985), and the Northern and Eastern Arms (infalling ionized gas that has been stripped off of the CND or has originated outside the CND; Lo \& Claussen 1983; Roberts \& Goss 1993).

\subsection{The $\mathrm{SiO}(2-1)$ data cube}

The $\mathrm{SiO}(2-1)$ emission appears above the $3 \sigma$ level in a wide velocity range: from $-125 \mathrm{~km} \mathrm{~s}^{-1}$, where the emission shows up in the southwest (relative to the position of $\mathrm{Sgr} \mathrm{A}^{*}$ ) to $130 \mathrm{~km} \mathrm{~s}^{-1}$, where the emission disappears in the northeast. The $\mathrm{SiO}(2-1)$ morphology shows the kinematics of the rotating CND. The southern and northern lobes are located at the ends of the major axis with the maximum LOS velocities, and the gas with the lower radial velocities is located in the minor axis (Marshall et al. 1995). The velocity pattern of the CND can be clearly seen in Fig. 3 at the RA $=\left[-45^{\prime \prime}, 45^{\prime \prime}\right]$ cut. The derived velocity gradient is $\sim 2.2 \mathrm{~km} \mathrm{~s}^{-1} \operatorname{arcsec}^{-1}\left(\sim 60 \mathrm{~km} \mathrm{~s}^{-1} \mathrm{pc}^{-1}\right)$. The southern lobe is stronger than the northern one, as in the case of all the previously $\mathrm{HCN}$ observations. $\mathrm{SiO}$ also shows the extension to the southwest detected by Marshall et al. (1995) in $\mathrm{HCN}(3-2)$, which is very likely the southernmost part of the Western Streamer, as discussed later. The inclination of the CND traced by the $\mathrm{SiO}$ emission agrees with previous observations, with the major axis aligned along the Galactic plane.

Figure 5 shows a composition of the velocity-integrated maps of the $\mathrm{SiO}(2-1)$ emission at the extreme CND velocities, $[-50,-20] \mathrm{km} \mathrm{s}^{-1}$ and $[70,100] \mathrm{km} \mathrm{s}^{-1}$, and the HCN(4-3) interferometric map of the CND obtained by Montero-Castaño et al. (2009) smoothed to $15^{\prime \prime}$ resolution. This clearly shows that the $\mathrm{SiO}(2-1)$ emission completely traces the CND.

In the most extreme blueshifted velocity ranges $\left([-125,-50] \mathrm{km} \mathrm{s}^{-1}\right)$, the $\mathrm{SiO}(2-1)$ line presents maximum emission at three locations: $\left(-15^{\prime \prime},-30^{\prime \prime}\right),\left(95^{\prime \prime}, 25^{\prime \prime}\right)$, and $\left(-40^{\prime \prime},-70^{\prime \prime}\right)$. The first peak emission correspond to the southwest lobe of the CND (labeled as SW CND in Fig. 4; see also Fig. 5). The maximum at $\left(95^{\prime \prime}, 25^{\prime \prime}\right)$ belongs to Cloud A (see Sect. 6.7), a small molecular cloud that seems to be isolated from the surrounding molecular gas (see also the declination-velocity plot at $\mathrm{RA}=\left[45^{\prime \prime}, 105^{\prime \prime}\right]$ in Fig. 3). In the $[-95,-50] \mathrm{km} \mathrm{s}^{-1}$ panel of Fig. 4 we can appreciate that this cloud is located inside the eastern edge of the SNR Sgr A East. The last maximum $\left(-40^{\prime \prime},-70^{\prime \prime}\right)$, which achieves its highest intensity in the velocity range $[-50,-20] \mathrm{km} \mathrm{s}^{-1}$, is also related to this SNR, as seems to border its southwestern region (labeled as Western St. South in Fig. 4). $\mathrm{HCO}^{+}(1-0)$ and $\mathrm{HCN}(1-0)$ emission has also been detected at this location by Wright et al. (2001). They attribute the emission to a continuation of the CND or to a high-velocity streamer. However, our data suggest that this emission could belong to the southernmost part of the Western Streamer. It appears in the declination-velocity maps (panel labeled RA $=\left[-105^{\prime \prime},-45^{\prime \prime}\right]$ in Fig. 3) as a coherent structure with a constant velocity gradient $\left(\sim 0.6 \mathrm{~km} \mathrm{~s}^{-1} \operatorname{arcsec}{ }^{-1}=17 \mathrm{~km} \mathrm{~s}^{-1} \mathrm{pc}^{-1}\right)$.

Between -50 and $10 \mathrm{~km} \mathrm{~s}^{-1}$, the gas seems to connect the southwestern and northeastern regions, surrounding the Sgr A* position. However, this feature is very likely the result of the superposition of several previously identified molecular features. The emission closer to $\mathrm{Sgr} \mathrm{A}^{*}$ in projection comes from 
M. A. Amo-Baladrón et al.: Mapping the surrounding of Sgr A*

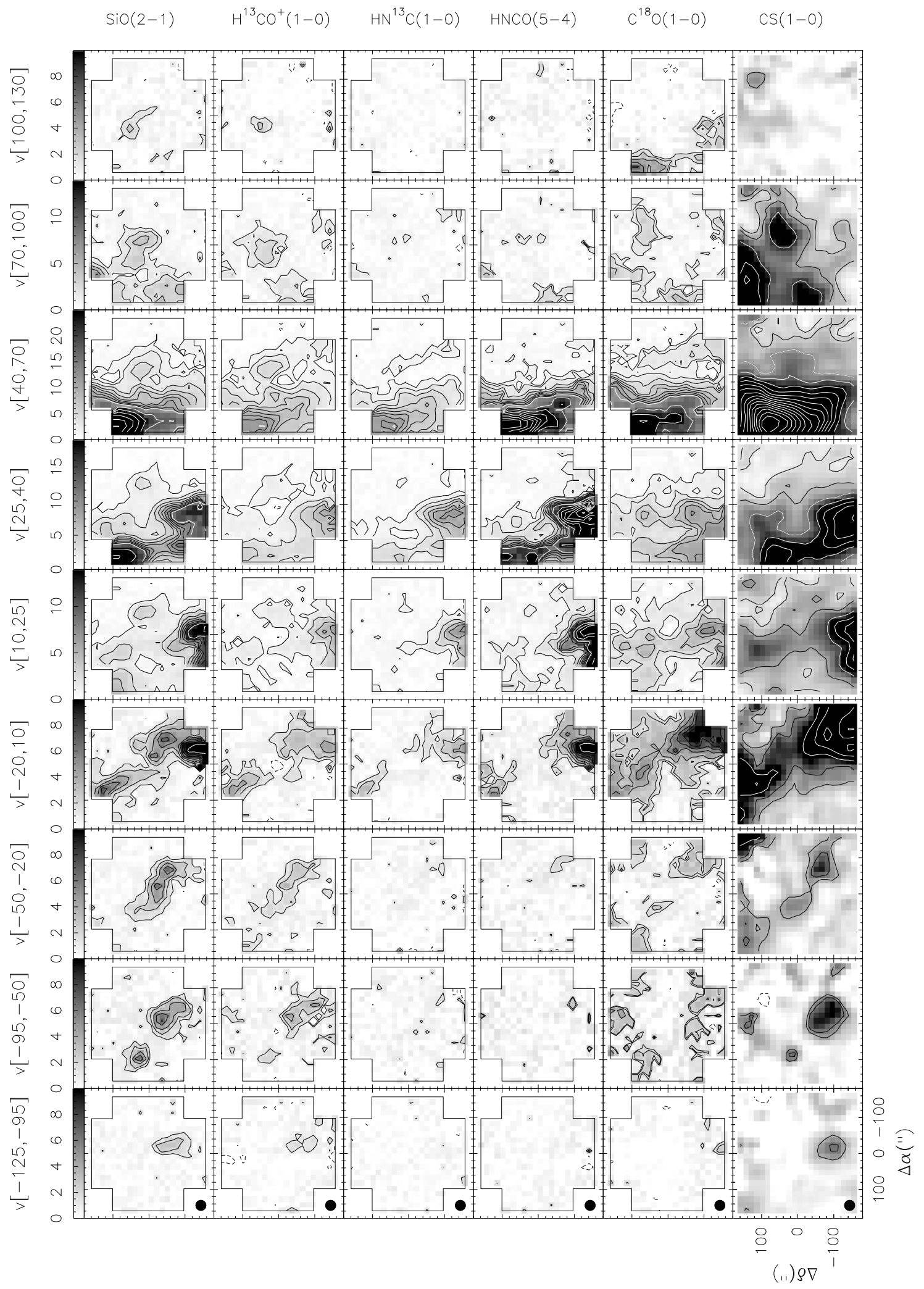

Fig. 2. Selected velocity-integrated maps for the different molecules observed at the Sgr A complex. Maps of the CS(1-0) line (Tsuboi et al. 1999) are displayed at the bottom. Each column corresponds to the same velocity range, indicated at the top of each column. The wedge at the top shows the intensity gray scale for each column. Contour levels for the integrated-velocity emission (in the $T_{\mathrm{A}}^{*}$ scale) of all the molecular lines, except CS(1-0), are $-3 \sigma$ (dashed contour), $3 \sigma$, from 2.0 to 12 in steps of $1.5 \mathrm{~K} \mathrm{~km} \mathrm{~s}^{-1}$ and from 12 in steps of $6 \mathrm{~K} \mathrm{~km} \mathrm{~s}^{-1}$. Contour levels for the velocity-integrated $\mathrm{CS}(1-0)$ emission are $-3 \sigma$ (dashed contour), $3 \sigma$, and from 6 in steps of $6 \mathrm{~K} \mathrm{~km} \mathrm{~s}^{-1}$. White contours are from $12 \mathrm{~K} \mathrm{~km} \mathrm{~s}{ }^{-1}$. The $3 \sigma$ levels corresponding to the 15,30 , and $45 \mathrm{~km} \mathrm{~s}^{-1}$ wide velocity ranges are $0.7,1.0$, and $1.2 \mathrm{~K} \mathrm{~km} \mathrm{~s}^{-1}$ for the $\mathrm{SiO}(2-1), \mathrm{H}^{13} \mathrm{CO}^{+}(1-0)$, and $\mathrm{HN}^{13} \mathrm{C}(1-0)$ maps; $1.0,1.3$, and $1.7 \mathrm{~K} \mathrm{~km} \mathrm{~s}^{-1}$ for the $\mathrm{HNCO}(5-4)$ and $\mathrm{C}^{18} \mathrm{O}(1-0)$ maps; and 2.6, 3.7, and $4.5 \mathrm{~K} \mathrm{~km} \mathrm{~s}^{-1}$ for the CS(1-0) maps. The beam size $\left(30^{\prime \prime}\right)$ is shown in the bottom-left corner of the first column panels. Sgr A* is the origin for the offset coordinates. 


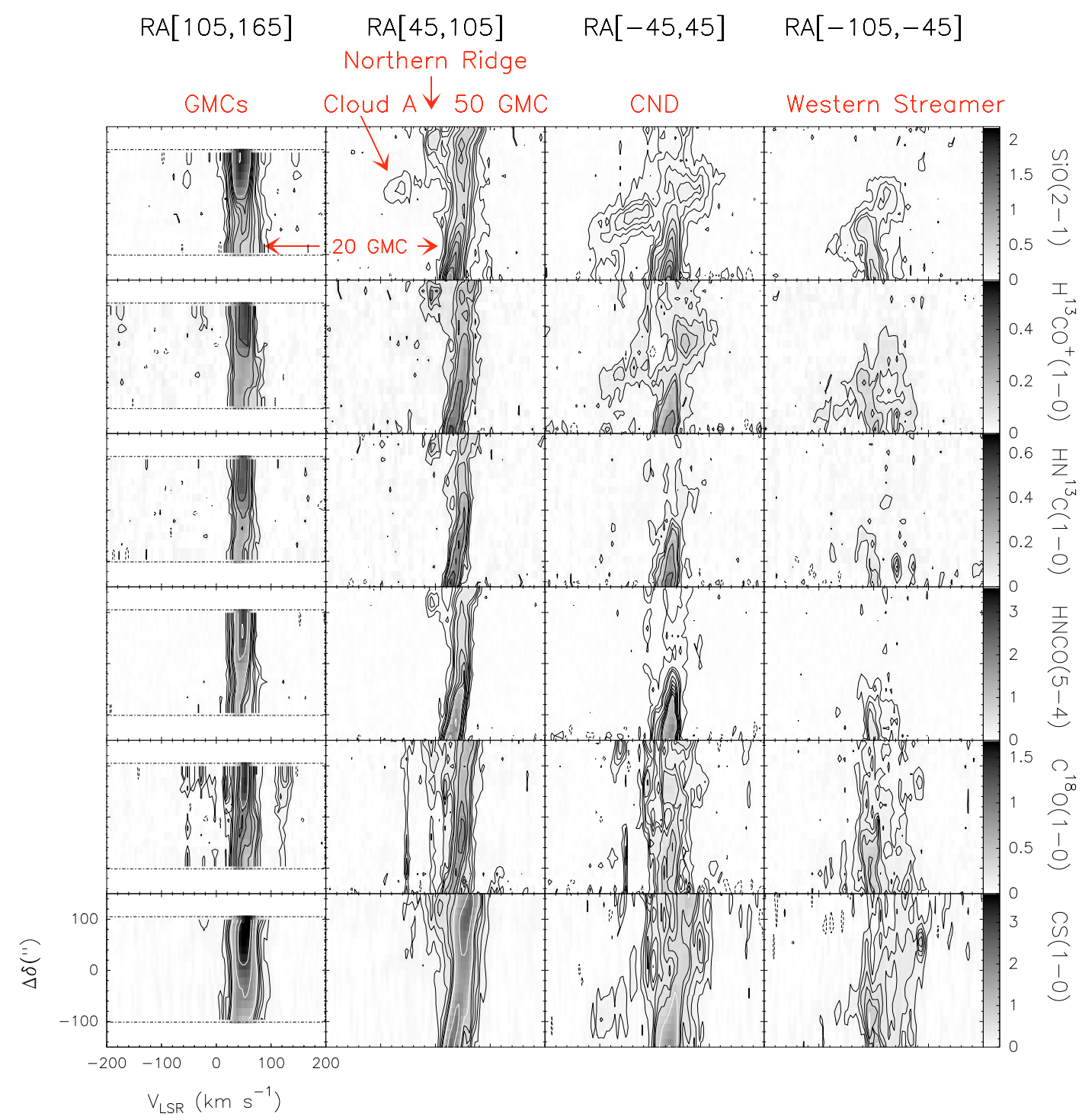

Fig. 3. Declination-velocity maps for the different molecules observed at the Sgr A complex. The $\Delta \delta$ - $v$ maps have been averaged in the right ascension ranges shown at the top of each column. Black contour levels are -3 (dashed contour), 3, 6, 9, and 12 $\sigma$. The following contours are in steps of $9 \sigma$ until $1 \mathrm{~K}$. In the case of the CS(1-0) maps, black contour levels are -3 (dashed contour), 3, 6, 9, 12, and 15 $\sigma$. The following contours are in steps of $12 \sigma$. White contours begin from $1 \mathrm{~K}$ and are in steps of $1 \mathrm{~K}$. The $3 \sigma$ levels corresponding to the $60^{\prime \prime}$ and $90^{\prime \prime}$ wide right ascension ranges are 0.04 and $0.03 \mathrm{~K}$ for the $\mathrm{SiO}(2-1), \mathrm{H}^{13} \mathrm{CO}^{+}(1-0)$, and $\mathrm{HN}^{13} \mathrm{C}(1-0)$ maps; 0.06 and $0.04 \mathrm{~K}$ for the $\mathrm{HNCO}(5-4)$ and $\mathrm{C}^{18} \mathrm{O}(1-0)$ maps; and 0.15 and $0.12 \mathrm{~K}$ for the $\mathrm{CS}(1-0)$ maps. The wedge on the right shows the intensity gray scale for each molecular line.

the eastern region of the CND (Jackson et al. 1993; Marshall et al. 1995). In previous HCN(1-0) single-dish observations, this part of the CND was absent because HCN emission was affected by self-absorption (Marshall et al. 1995). Even with the singledish resolution, $\mathrm{SiO}$ emission perfectly traces the eastern region, although emission here is weaker than in the rest of the CND. Finally, the emission in this velocity range located in the northeastern quadrant $\left(\Delta \delta>40^{\prime \prime}\right)$ comes from the Northern Ridge and borders, in projection, the northern edge of the SNR Sgr A East. Emission from the western side $\left(\Delta \alpha<-40^{\prime \prime}\right)$ corresponds to the Western Streamer, whereas emission from the south comes from the northern part of the $20 \mathrm{~km} \mathrm{~s}^{-1} \mathrm{GMC}$, both features bordering the western and southwestern edges of this SNR (see Fig. 4).

In the velocity range $[10,40] \mathrm{km} \mathrm{s}^{-1}$, we can see the northern region of the $20 \mathrm{~km} \mathrm{~s}^{-1}$ GMC (the Southern Streamer) and $50 \mathrm{~km} \mathrm{~s}^{-1}$ GMCs at east, together with the northern half of the Western Streamer, which peaks at $\left(-60^{\prime \prime}, 20^{\prime \prime}\right)$, west of the
CND (labeled as Western St. North in Fig. 4). At $-5 \mathrm{~km} \mathrm{~s}^{-1}$, the $20 \mathrm{~km} \mathrm{~s}^{-1} \mathrm{GMC}$ begins to appear at the south. As the velocity increases, the molecular emission moves to the east to form other well-known features: the Molecular Ridge, a connection between the two most massive clouds of the Sgr A complex, the $20 \mathrm{~km} \mathrm{~s}^{-1}$ and $50 \mathrm{~km} \mathrm{~s}^{-1}$ GMCs. These three molecular features surround a cavity where the $\mathrm{SiO}(2-1)$ emission has low intensity between 20 to $35 \mathrm{~km} \mathrm{~s}^{-1}$. At $70 \mathrm{~km} \mathrm{~s}^{-1}$, the $50 \mathrm{~km} \mathrm{~s}^{-1}$ GMC finally disappears. The $50 \mathrm{~km} \mathrm{~s}^{-1}$ GMC is located on the eastern border of the SNR Sgr A East (see Fig. 4), whereas the molecular gas at the Southern Streamer could be affected by the interaction of two expanding shells: Sgr A East and G 359.92-0.09 (Coil \& Ho 2000).

In the velocity range $[40,70] \mathrm{km} \mathrm{s}^{-1}$, we can see another small cloud peaking at $\left(-10^{\prime \prime}, 20^{\prime \prime}\right)$, just with a small offset east from the northern half of the Western Streamer. If we compare with the HCN(4-3) map of Montero-Castaño et al. (2009), we 
M. A. Amo-Baladrón et al.: Mapping the surrounding of Sgr A*

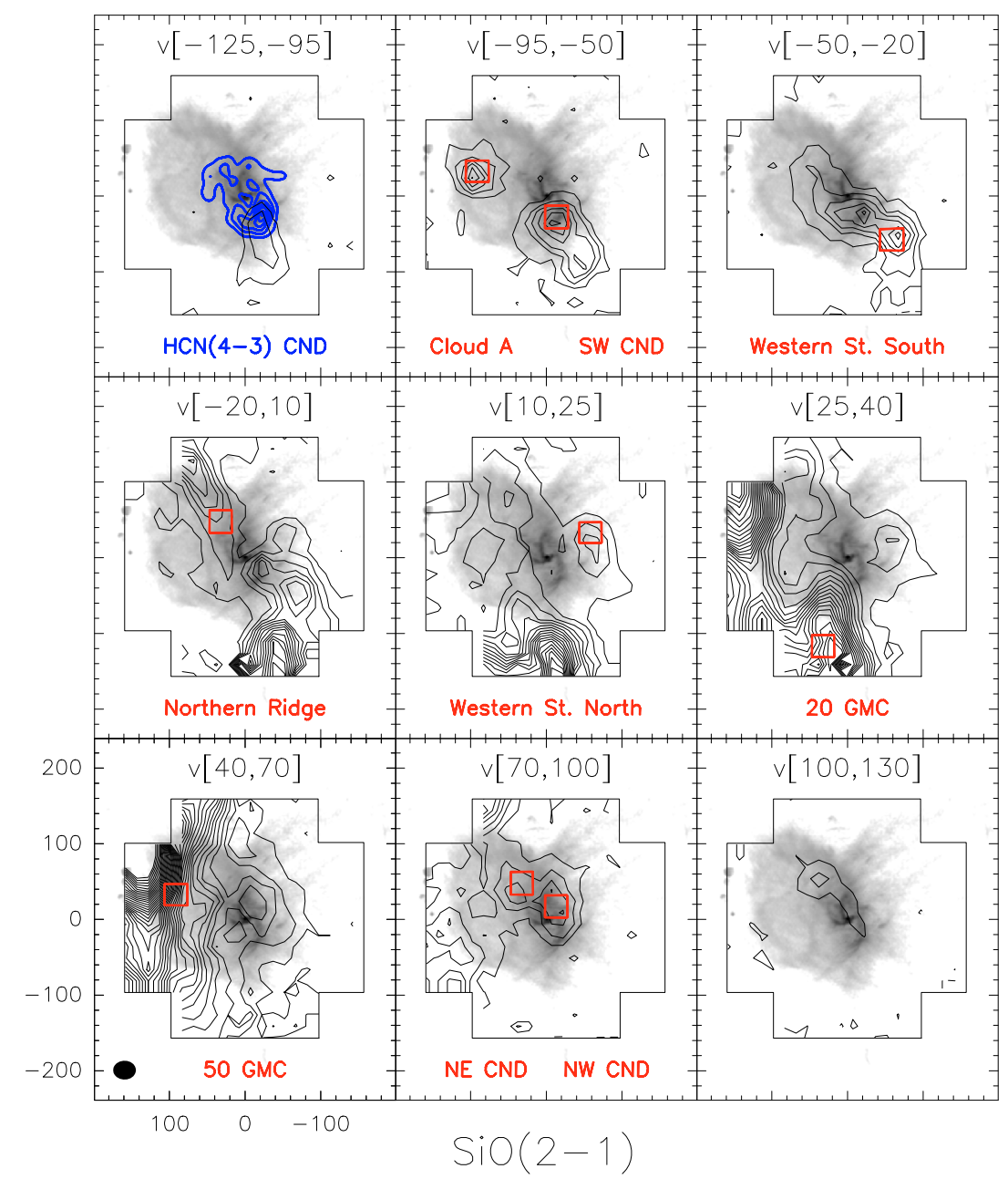

Fig. 4. Selected velocity-integrated maps of $\mathrm{SiO}(2-1)$ emission superimposed on the radio continuum image of Yusef-Zadeh \& Morris (1987) at $6 \mathrm{~cm}$ in gray scale. We can appreciate the thermal feature Sgr A West, also known as the Minispiral, in the center of the image. The first two level contours are $-0.70 .7,-1.01 .0$, and $-1.21 .2 \mathrm{~K} \mathrm{~km} \mathrm{~s}^{-1}$ for integrated velocities 15,30 , and $45 \mathrm{~km} \mathrm{~s}^{-1}$, respectively. Steps between levels are $1 \mathrm{~K} \mathrm{~km} \mathrm{~s}^{-1}$. Open squares indicate the spatially integrated areas where spectra of Fig. 6 were obtained, which are associated with the main identified molecular features, whose names are indicated at the bottom of each corresponding panel. In the first panel, we also show the HCN(4-3) map of the CND of Montero-Castaño et al. (2009) smoothed to $15^{\prime \prime}$ in thick blue contours.

can conclude that this cloud could be part of the northwestern region of the CND (labeled as NW CND in Fig. 4). In the velocity range $[70,100] \mathrm{km} \mathrm{s}^{-1}$, the emission from this cloud moves to the east (maximum at $\left.\left(40^{\prime \prime}, 35^{\prime \prime}\right)\right)$ showing a very good correlation with the northeastern region of the CND.

Finally, in the highest velocity range $\left([100,130] \mathrm{km} \mathrm{s}^{-1}\right)$, the maximum of the emission is located at the north of the $\mathrm{CND}$, at $\left(35^{\prime \prime}, 55^{\prime \prime}\right)$. In summary, $\mathrm{SiO}(2-1)$ emission traces all previously identified molecular structures within the central $12 \times 12 \mathrm{pc}^{2}$ region.

\subsection{The $\mathrm{H}^{13} \mathrm{CO}^{+}(1-0)$ data cube}

The $\mathrm{H}^{13} \mathrm{CO}^{+}(1-0)$ emission covers the same velocity range as the $\mathrm{SiO}(2-1)$ emission and nicely follows its morphology, except for the lower intensity of the $\mathrm{H}^{13} \mathrm{CO}^{+}$emission and the absorption features that appear toward Sgr A*. Two absorption features are observed (Fig. A.2): one narrow feature centered at $-52 \mathrm{~km} \mathrm{~s}^{-1}$ arising from the $3-\mathrm{kpc}$ spiral arm (Lindqvist et al. 1995) and observed in emission in the $\mathrm{C}^{18} \mathrm{O}(1-0)$ maps (see Sect. 3.5); and a wide feature arising from local gas with a likely contribution from gas within the nuclear region, since the
$\mathrm{SiO}(2-1)$ emission also shows a decrease in its intensity across this velocity range.

\subsection{The $H N^{13} C(1-0)$ data cube}

The $\mathrm{HN}^{13} \mathrm{C}(1-0)$ emission is weaker than that of $\mathrm{H}^{13} \mathrm{CO}^{+}(1-0)$ and $\mathrm{SiO}(2-1)$. Most of its emission is concentrated in the GMCs and in the Molecular Ridge, going from -20 to $70 \mathrm{~km} \mathrm{~s}^{-1}$. There is also low-intensity emission coming from the Northern Ridge visible in the velocity range $[-20,10] \mathrm{km} \mathrm{s}^{-1}$; however, the eastern border of the CND is not traced by $\mathrm{HN}^{13} \mathrm{C}$. In contrast, its western side seems to be detected at the noise level in the velocity ranges between $[40,100] \mathrm{km} \mathrm{s}^{-1}$. For the $-82 \mathrm{~km} \mathrm{~s}^{-1}$ velocity-channel map (Fig. A.3), we can see very low-intensity emission coming from Cloud A, however, in the corresponding velocity-integrated map there is no trace of this cloud. At the velocity ranges between $[10,40] \mathrm{km} \mathrm{s}^{-1}$ there is low intensity emission from the northern half of the Western Streamer, as well as from its southern half in the velocity ranges between $[-95,10] \mathrm{km} \mathrm{s}^{-1}$. 

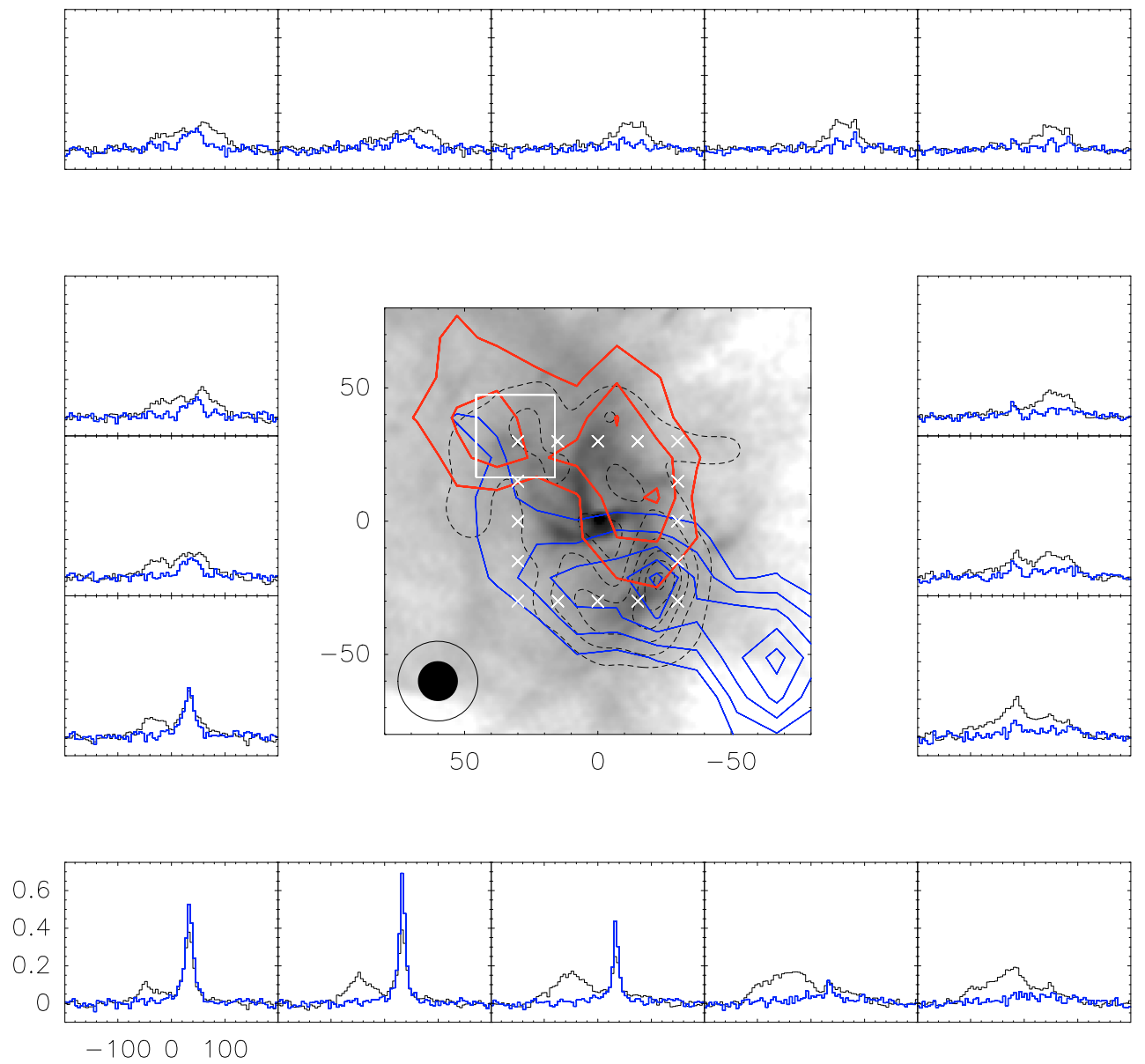

Fig. 5. Composition of the velocity-integrated maps of the $\mathrm{SiO}(2-1)$ emission at extreme velocities (velocity ranges between $[-50,-20] \mathrm{km} \mathrm{s}^{-1}$ in thin blue contours and $[70,100] \mathrm{km} \mathrm{s}^{-1}$ in thick red ones), superimposed on the radio continuum image of Yusef-Zadeh \& Morris (1987) at $6 \mathrm{~cm}$ in gray scale. Black dashed contours correspond to the HCN(4-3) interferometric map of the CND (Montero-Castaño et al. 2009) smoothed to $15^{\prime \prime}$ resolution. The $\mathrm{SiO}(2-1)$ contour levels goes from $2 \mathrm{~K} \mathrm{~km} \mathrm{~s}^{-1}$ in steps of $1 \mathrm{~K} \mathrm{~km} \mathrm{~s}^{-1}$. The white crosses indicate the centers of the $30^{\prime \prime} \times 30^{\prime \prime}$ squares where the shown spectra of $\mathrm{SiO}$ (black lines) and HNCO (thick blue lines) have been obtained. The beam size of the $\mathrm{SiO}(2-1)$ and $\mathrm{HCN}(4-3)$ emissions are shown as open and filled circles, respectively, in the bottom-left corner of the map.

\subsection{The HNCO(5-4) data cube}

This molecular line is the one that shows emission in the narrowest velocity range, only from -30 to $85 \mathrm{~km} \mathrm{~s}^{-1}$ in the velocity-channel maps (Fig. A.4). The HNCO emission is very intense only in the 20 and $50 \mathrm{~km} \mathrm{~s}^{-1}$ GMCs, and in the Molecular Ridge that connects both clouds. It is remarkable that $\mathrm{HNCO}(5-4)$ emission is the strongest of all the emission lines we have mapped, including $\mathrm{C}^{18} \mathrm{O}$. Outside these dense GMCs, the $\mathrm{HNCO}$ emission quickly drops. Between -25 and $-5 \mathrm{~km} \mathrm{~s}^{-1}$, we can appreciate the HNCO counterparts of the Northern Ridge and the $20 \mathrm{~km} \mathrm{~s}^{-1}$ GMC. However, as the molecular gas approaches the central position, it disappears. We do not detect the $\mathrm{HNCO}$ emission from Cloud A observed in other molecular lines at negative velocities. There is, however, a very low-level emission from the southern and northern parts of the Western Streamer in the velocity ranges between $[-95,40] \mathrm{km} \mathrm{s}^{-1}$. Finally, the western side of the CND seems to be detected in the velocity range $[70,100] \mathrm{km} \mathrm{s}^{-1}$.

\subsection{The $C^{18} O(1-0)$ data cube}

The $\mathrm{C}^{18} \mathrm{O}(1-0)$ emission ranges from -80 to $95 \mathrm{~km} \mathrm{~s}^{-1}$ in the velocity-channel maps (Fig. A.5). However, only for velocities $\geq-30 \mathrm{~km} \mathrm{~s}^{-1}$ its emission seems to resemble the morphology observed in other molecular lines. We also find very low-intensity emission at around $170 \mathrm{~km} \mathrm{~s}^{-1}$ detectable in the spatially-averaged spectrum over the whole region, probably arising from the expanding molecular ring (Lindqvist et al. 1995), which also appears in the CS(1-0) averaged spectrum. Between -80 to $-55 \mathrm{~km} \mathrm{~s}^{-1}$, the emission comes from the north, with no detected counterparts in the other molecules. Most of the narrow emission only seen in the $-52 \mathrm{~km} \mathrm{~s}^{-1}$ channel map is foreground, arising from the 3-kpc spiral arm (Lindqvist et al. 1995). This feature is observed in absorption in the $\mathrm{H}^{13} \mathrm{CO}^{+}(1-0)$ maps (see Sect. 3.2). The Northern Ridge, as well as gas surrounding $\mathrm{Sgr}^{*}$ with stronger emission at its western side, can be detected between -20 and $10 \mathrm{~km} \mathrm{~s}^{-1}$. If we compare emission at these velocities with the $\mathrm{SiO}(2-1)$, we notice that the $\mathrm{C}^{18} \mathrm{O}(1-0)$ emission is shifted toward the west. There is strong emission arising from the 20 and $50 \mathrm{~km} \mathrm{~s}^{-1}$ GMCs, and the Molecular Ridge, but we cannot clearly distinguish the emission from the northern part of the Western Streamer and Cloud A. At the highest velocities, there is $\mathrm{C}^{18} \mathrm{O}(1-0)$ emission coming from the north, probably associated with the northwestern lobe of the CND, but more extended to the northwest than the $\mathrm{SiO}(2-1)$ and $\mathrm{H}^{13} \mathrm{CO}^{+}(1-0)$ emission at these velocities. The same happens to the $\mathrm{CS}(1-0)$ emission. 
From 100 to $130 \mathrm{~km} \mathrm{~s}^{-1}$, we find low-intensity emission that resembles the morphology of the Molecular Ridge and the two GMCs of the Sgr A complex. This emission could come from $\mathrm{SO}_{2}\left(17_{5,13}-18_{4,14}\right)$ (Lindqvist et al. 1995) and/or $\mathrm{NH}_{2} \mathrm{CHO}\left(5_{1,4}-4_{1,3}\right)$ (Jackson et al. 1984; Cummins et al. 1986). Cummins et al. (1986) found this line in their spectral survey of $\mathrm{Sgr} \mathrm{B} 2$ and considered that the $\mathrm{SO}_{2}$ line contributed with $10 \%$. We also consider that the $\mathrm{NH}_{2} \mathrm{CHO}$ line is the strongest one in this case. The upper-level energy of the $\mathrm{NH}_{2} \mathrm{CHO}$ transition is lower than the upper-level energy of the $\mathrm{SO}_{2}$ transition ( $19 \mathrm{~K}$ vs. $202 \mathrm{~K}$ ). Moreover, the central velocity of the $\mathrm{NH}_{2} \mathrm{CHO}$ line at the maximum of its emission is $45 \mathrm{~km} \mathrm{~s}^{-1}$, whereas the central velocity of the $\mathrm{SO}_{2}$ line is slightly redshifted $\left(56 \mathrm{~km} \mathrm{~s}^{-1}\right)$ with respect to the lines from the other species $\left(\simeq 49 \mathrm{~km} \mathrm{~s}^{-1}\right.$ on average). The origin of the $\mathrm{NH}_{2} \mathrm{CHO}$ molecule could be related to the HNCO origin. Bisschop et al. (2007) find a strong correlation between both species in their survey of molecules suspected to be produced by the grain surface chemistry at several selected highmass young stellar objects. This correlation suggests that HNCO and $\mathrm{NH}_{2} \mathrm{CHO}$ molecules share a similar formation mechanism, i.e. grain chemistry. The $\mathrm{NH}_{2} \mathrm{CHO}$ emission appears to be stronger in the $50 \mathrm{~km} \mathrm{~s}^{-1}$ GMC, a molecular cloud affected by shocks driven by the SNR Sgr A East, that could eject this molecule to the gas phase. In the following, we do not discuss this emission since it is rather weak, and it is only significantly detected toward the $50 \mathrm{~km} \mathrm{~s}^{-1}$ GMC.

\section{Physical conditions of the gas and column density estimates}

To estimate the column densities of the observed molecules, we selected several positions located at maxima of the $\mathrm{SiO}(2-1)$ emission. The spectra toward these positions can be seen in Fig. 6. These spectra show at least 9 different velocity components that can be fitted to Gaussian profiles, however, other components cannot be fitted. Moreover, in some cases it is not possible to separate contributions from the different molecular features that appear at the same position but in different velocity ranges using Gaussian profiles. Therefore, we derived the column densities of the observed molecules from integrated intensities in selected velocity ranges (see Table 2). These selected velocity ranges are shown as shadowed areas in Fig. 6, labeled with their associated molecular feature, and the location of most of these components in the $\alpha-\delta-v$ space is indicated in Fig. 4. We note that the components 20 GMC North and 50 GMC West are very likely contaminated with molecular gas from the CND.

The procedure used to derive the column densities is the following. We constrained combinations of the pair kinetic temperature $\left(T_{\mathrm{k}}\right)$ and volume density $\left(n_{\mathrm{H}_{2}}\right)$, together with the $\mathrm{SiO}$ column density $\left(N_{\mathrm{SiO}}\right)$, by matching the two observed transitions of $\mathrm{SiO}$ (two upper spectra in each panel of Fig. 6) using a large velocity gradient (LVG) excitation code. We explored a range of $T_{\mathrm{k}}$ from 30 to $200 \mathrm{~K}$, as derived from ammonia inversion lines by Hüttemeister et al. (1993) in several clouds of the GC and by Herrnstein \& Ho (2005) in the central 10-20 pc region. Both studies conclude that molecular clouds in the GC can be roughly characterized by a two-temperature gas distribution in which $75 \%$ of the column density comes from cool gas $(\sim 25 \mathrm{~K})$ and the remaining $25 \%$ from hot gas $(\sim 200 \mathrm{~K})$. Furthermore, Rodríguez-Fernández et al. (2001) find, from observations of $\mathrm{S}(0)$ to $\mathrm{S}(5) \mathrm{H}_{2}$ pure-rotational lines toward several molecular clouds of the GC, that warm gas $(\sim 150 \mathrm{~K})$ represents the

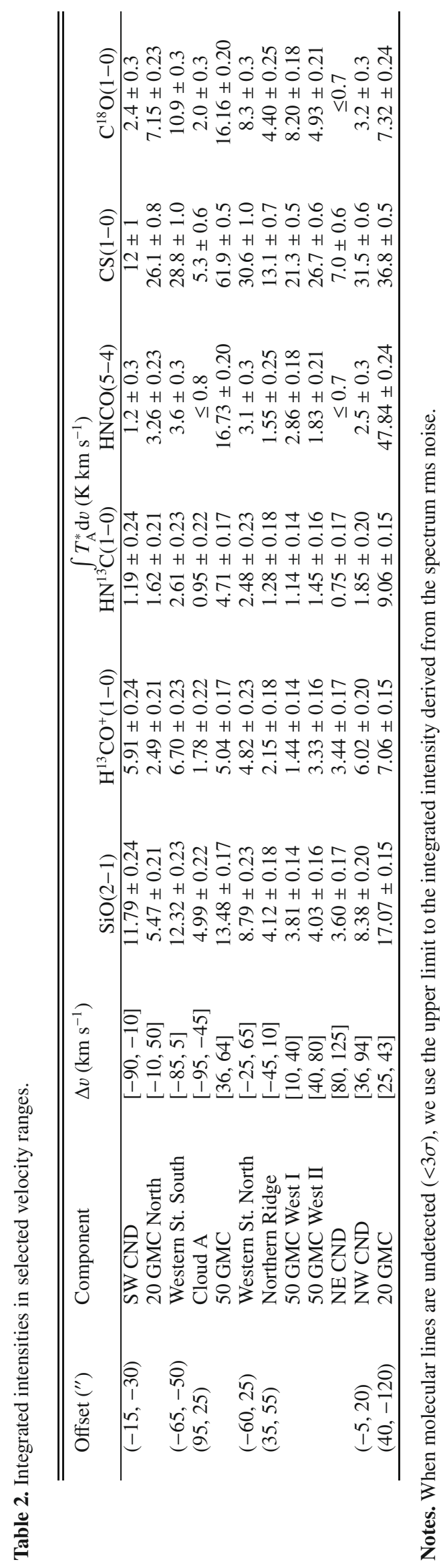

A54, page 9 of 29 


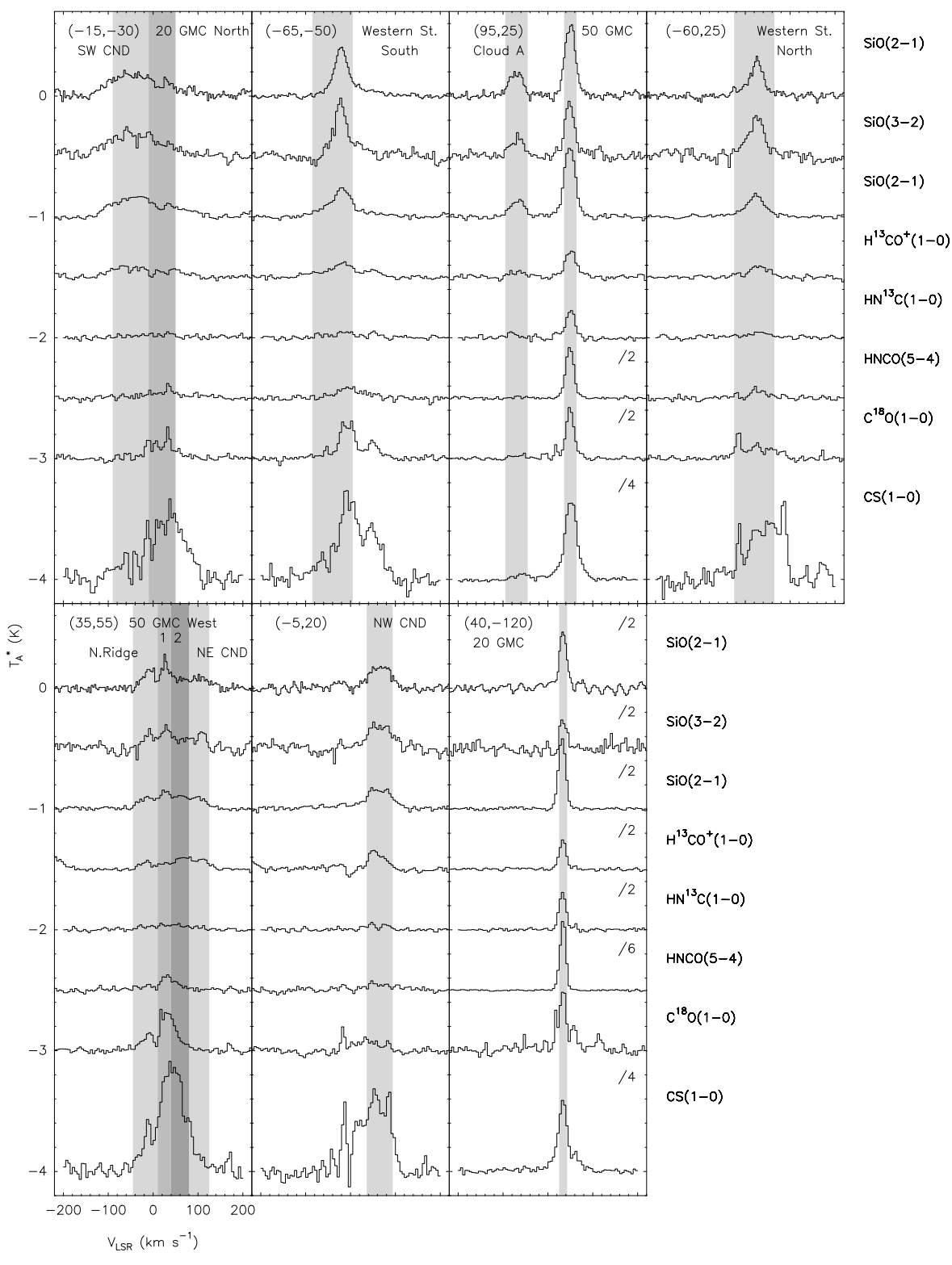

Fig. 6. Spectra of the observed transitions taken at 7 selected positions (marked with open squares in Fig. 4). The upper $\mathrm{SiO}(2-1)$ and the $\mathrm{SiO}(3-2)$ spectra were observed simultaneously at the offset positions respect to Sgr A* (in arcseconds) shown in the upper-left corner of each panel. The other $\mathrm{SiO}(2-1)$ spectra, together with the other molecular line spectra, were extracted from their respective data cubes from squares of area $30^{\prime \prime} \times 30^{\prime \prime}$. Shadowed areas represent the selected velocity ranges where integrated intensities of the observed molecular lines were obtained, labeled with the names of their associated features. The intensity of some transitions has been divided by a factor shown in the upper-right corner of the corresponding spectrum. $\sim 30 \%$ of the gas traced by low- $J$ CO isotopes. Then, we modeled the intensity of the other molecular lines (of $\operatorname{HNCO}(5-4)$ and $\mathrm{C}^{18} \mathrm{O}(1-0)$ lines using the RADEX LVG excitation code; van der Tak et al. 2007) with the previously derived physical condition pairs $\left(T_{\mathrm{k}}\right.$ and $\left.n_{\mathrm{H}_{2}}\right)$, in order to obtain their column densities. As all the observed transitions, except $\mathrm{C}^{18} \mathrm{O}(1-0)$, have similar dipole moments (from 1.60 to $3.89 \mathrm{D}$ ), we can assume that all transitions sample the same molecular gas (i.e., gas with similar physical conditions). However, for the $\mathrm{C}^{18} \mathrm{O}(1-0)$ line $(0.11 \mathrm{D})$, the derived column density values should be taken with caution.

In Table 3 we present the results of this analysis for a $T_{\mathrm{k}}$ of $50 \mathrm{~K}$. Raising the kinetic temperature by a factor of 4 has almost no effect on the excitation temperature and column density of $\mathrm{SiO}$, but it decreases the volume density by a factor of $\sim 3$, except for the $20 \mathrm{~km} \mathrm{~s}^{-1} \mathrm{GMC}$, where the volume density decreases by a factor of 4 . The derived $n_{\mathrm{H}_{2}}$ values at $T_{\mathrm{k}}=50 \mathrm{~K}$ are very similar for all the gas components, $(1.5-5) \times 10^{5} \mathrm{~cm}^{-3}$, except for the $20 \mathrm{~km} \mathrm{~s}^{-1} \mathrm{GMC}$, where the volume density shows the minimum value $\left(\sim 4 \times 10^{4} \mathrm{~cm}^{-3}\right)$.
The $\mathrm{SiO}$ emission is optically thin, showing maximun optical depths of 0.5 toward the 20 and $\sim 0.15$ toward $50 \mathrm{~km} \mathrm{~s}^{-1}$ GMCs. The $\mathrm{SiO}$ transitions are subthermally excited, with $T_{\mathrm{ex}}$ ranging from $4 \mathrm{~K}$ at the $20 \mathrm{~km} \mathrm{~s}^{-1}$ GMC to $12 \mathrm{~K}$ in the northern part of the Western Streamer, in agreement with previous estimates in the GC (Hüttemeister et al. 1998). The SiO column densities, $0.6-6 \times 10^{13} \mathrm{~cm}^{-2}$, show less variation than the HNCO column densities, $\leq 0.8-50 \times 10^{13} \mathrm{~cm}^{-2}$.

HNCO column density presents the strongest contrast, with a difference of a factor of $\geq 60$ between Cloud $A$ and the $20 \mathrm{~km} \mathrm{~s}^{-1}$ GMC. HNCO excitation temperatures show larger variations, depending on the volume density. Except for the $20 \mathrm{~km} \mathrm{~s}^{-1}$ GMC, where the excitation temperature ranges between 9 and $15 \mathrm{~K}$, the $\mathrm{HNCO}$ excitation temperatures are in general $\geq 40 \mathrm{~K}$. These values agree with the rotational temperatures derived by Martín et al. (2008) using the rotational diagrams obtained from several HNCO transitions. They obtained $T_{\text {rot }}=35 \pm 12 \mathrm{~K}$ and $T_{\text {rot }}=13.6 \pm 0.2 \mathrm{~K}$ in their positions $\left(-30^{\prime \prime},-30^{\prime \prime}\right)$ and $\left(-15^{\prime \prime},-215^{\prime \prime}\right)$ relative to $\mathrm{Sgr} \mathrm{A}^{*}$, respectively. The first position almost coincides with our $\left(-15^{\prime \prime},-30^{\prime \prime}\right)$ 


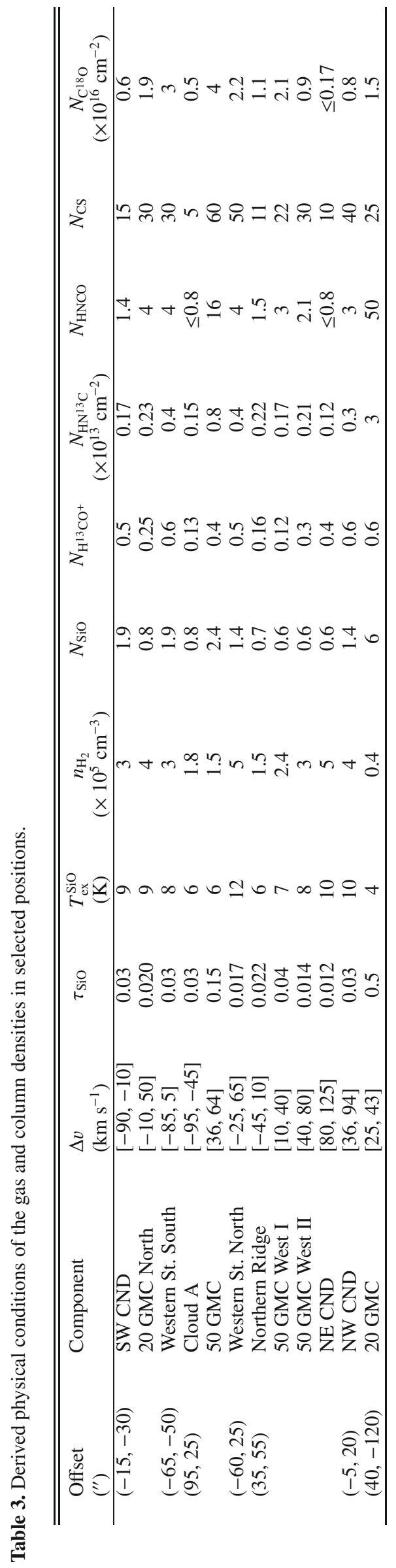

position, whereas the second is located at the core of the $50 \mathrm{~km} \mathrm{~s}^{-1}$ GMC. Therefore, our results share a similar trend, that is, the lowest HNCO excitation temperatures are shown toward the cores of the GMCs. HNCO column densities show almost no variation for the different $T_{\mathrm{k}}$ and $n_{\mathrm{H}_{2}}$ pair values derived from the $\mathrm{SiO}$ transitions. The largest variation is found for the $20 \mathrm{~km} \mathrm{~s}^{-1}$ GMC position with only a factor 1.6 more at $200 \mathrm{~K}$ than at $50 \mathrm{~K}$. The HNCO emission is optically thin in all the cases $(\leq 0.2)$.

In general, uncertainties in the column densities due to calibration (assuming a conservative 20\%), and rms noise in the spectra are lower than a factor of 2. As for $\mathrm{HNCO}$ and $\mathrm{SiO}$, changing the kinetic temperature from 50 to $200 \mathrm{~K}$ has almost no effect on the $\mathrm{H}^{13} \mathrm{CO}^{+}, \mathrm{HN}^{13} \mathrm{C}$, and $\mathrm{CS}$ column densities. The largest variation is also found for the $20 \mathrm{~km} \mathrm{~s}^{-1} \mathrm{GMC}$ position, with a factor of 1.2 and around 1.7 larger at $200 \mathrm{~K}$ than at $50 \mathrm{~K}$ for $\mathrm{CS}$ and the other species. The $\mathrm{C}^{18} \mathrm{O}$ column densities present larger differences with kinetic temperature, with factors up to 3. This higher sensitivity to volume density is expected, as $\mathrm{C}^{18} \mathrm{O}$ is the molecule with the lowest critical density among the species studied in this paper. Therefore, we conclude that our column density estimates for $\mathrm{SiO}, \mathrm{H}^{13} \mathrm{CO}^{+}, \mathrm{HN}^{13} \mathrm{C}, \mathrm{HNCO}$, and $\mathrm{CS}$ could present uncertainties of up to a factor of 2 .

\section{Integrated intensity maps and fractional abundances}

To derive the fractional abundances of the observed species, we need to evaluate the hydrogen column density at the selected positions. As mentioned before, $\mathrm{C}^{18} \mathrm{O}$ is not the best tracer because it samples molecular gas biased toward low hydrogen volume densities, and it is strongly affected by gas along the LOS toward the GC. Alternatively, CS is more likely arising from the same high-density molecular gas as the other observed molecules, and it is moderately affected by any particular chemistry taking place at the central 12 parsecs. It is only marginally enhanced in UV (Goicoechea et al. 2006; Martín et al. 2008) and shock-dominated environments (Requena-Torres et al. 2006). However, the drawback is that CS(1-0) could be moderately affected by opacity.

To elucidate the effects of CS opacity in our estimation of the fractional abundances, we made integrated intensity ratio maps relative to the CS(1-0) emission (Fig. 7). Additionally, we present intensity ratio maps made in the same selected velocity ranges as Fig. 2 (Fig. B.1). As we can see in Figs. 7 and B.1, the $\mathrm{C}^{18} \mathrm{O}(1-0) / \mathrm{CS}(1-0)$ ratio is rather uniform. The highest values are found at negative velocities, very likely associated with foreground molecular gas, and the lowest values at positive velocities associated with the northern part of the CND. These lower values indicate that the molecular gas is denser in the CND than in the surrounding GMCs (see Table 3). Therefore, CS(1-0) emission does not seem to be severely affected by optical depth effects.

Using CS as a high-density molecular gas tracer and assuming a CS fractional abundance of $X_{\mathrm{CS}}=N_{\mathrm{CS}} / N_{\mathrm{H}_{2}}=10^{-8}$ (Irvine et al. 1987) for the GC, we derive hydrogen column densities between $0.5-6 \times 10^{22} \mathrm{~cm}^{-2}$. As already mentioned, Table 4 and Fig. 8 show that $\mathrm{C}^{18} \mathrm{O}$ is the molecule that presents the smallest variation in its fractional abundance, with only a difference of a factor of 6. On the other hand, HNCO is the molecule that presents the largest variation of a factor of 28, with the highest values toward the cores of the GMCs, and the lowest ones toward the positions associated with the CND. Conversely, $\mathrm{SiO}$ presents 
Table 4. Fractional abundances.

\begin{tabular}{llccccc}
\hline \hline $\begin{array}{l}\text { Offset } \\
\left({ }^{\prime \prime}\right)\end{array}$ & Component & $X_{\mathrm{SiO}}$ & $X_{\mathrm{H}^{13} \mathrm{CO}^{+}}$ & $\begin{array}{c}X_{\mathrm{HN}^{13} \mathrm{C}} \\
\left(\times 10^{-9}\right)\end{array}$ & $X_{\mathrm{HNCO}}$ & $\begin{array}{c}X_{\mathrm{C}^{18} \mathrm{O}} \\
\left(\times 10^{-7}\right)\end{array}$ \\
\hline$(-15,-30)$ & SW CND & 1.3 & 0.4 & 0.12 & 1.0 & 4 \\
& 20 GMC North & 0.24 & 0.07 & 0.07 & 1.1 & 5 \\
$(-65,-50)$ & Western St. South & 0.6 & 0.18 & 0.11 & 1.2 & 8 \\
$(95,25)$ & Cloud A & 1.8 & 0.3 & 0.3 & $\leq 1.7$ & 11 \\
& 50 GMC & 0.4 & 0.06 & 0.14 & 3 & 7 \\
$(-60,25)$ & Western St. North & 0.3 & 0.11 & 0.08 & 0.9 & 5 \\
$(35,55)$ & Northern Ridge & 0.6 & 0.15 & 0.20 & 1.4 & 10 \\
& 50 GMC West I & 0.3 & 0.05 & 0.08 & 1.4 & 9 \\
& 50 GMC West II & 0.20 & 0.09 & 0.07 & 0.7 & 3 \\
& NE CND & 0.6 & 0.4 & 0.12 & $\leq 0.8$ & $\leq 1.8$ \\
$(-5,20)$ & NW CND & 0.3 & 0.14 & 0.06 & 0.7 & 1.9 \\
$(40,-120)$ & 20 GMC & 2.4 & 0.25 & 1.3 & 19 & 6 \\
\hline
\end{tabular}

Notes. Fractional abundances with respect to $\mathrm{H}_{2}$, derived from the CS column density assuming $X_{\mathrm{CS}}=10^{-8}$ for the GC.

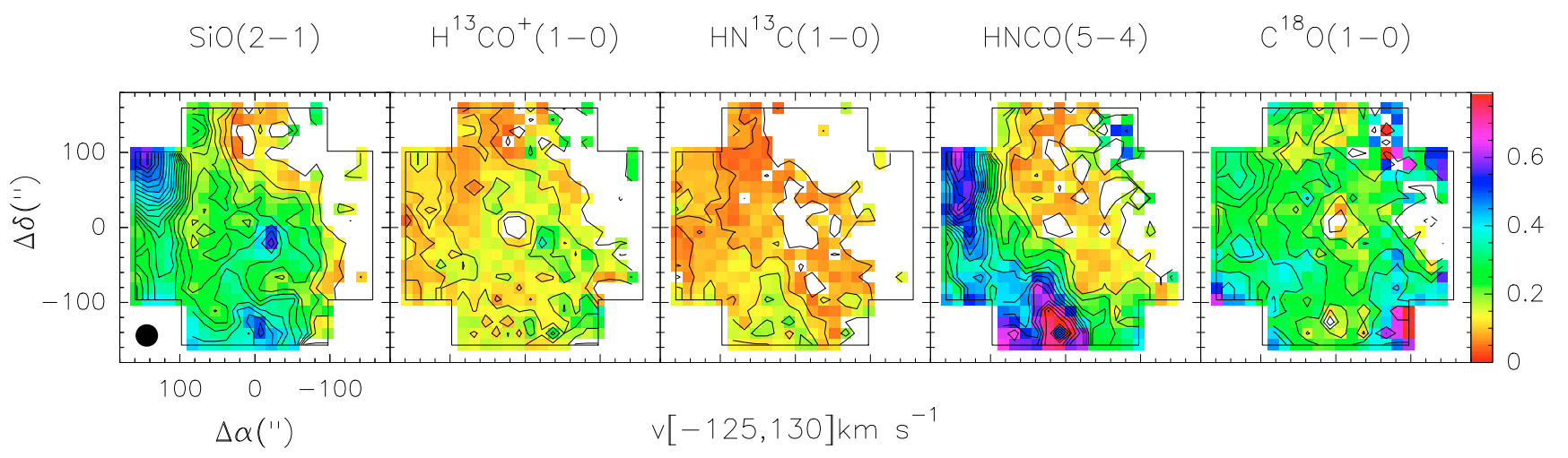

Fig. 7. $\mathrm{X} / \mathrm{CS}(1-0)$ intensity ratios in the full velocity range shown at the bottom of the figure. $X$ stands for $\mathrm{SiO}(2-1), \mathrm{H}^{13} \mathrm{CO}^{+}(1-0), \mathrm{HN}^{13} \mathrm{C}(1-0)$, $\mathrm{HNCO}(5-4)$, and $\mathrm{C}^{18} \mathrm{O}(1-0)$, whose emission is shown in contour levels. Intensity ratios have been derived considering only pixels with emission above the $3 \sigma$ level. The wedge to the right shows the color scale of the intensity ratios. Contour levels are $-3 \sigma$ (dashed contour) and from $3 \sigma$ in steps of $4 \sigma$ (3.9 and $5.2 \mathrm{~K} \mathrm{~km} \mathrm{~s}^{-1}$ for $\mathrm{SiO} / \mathrm{H}^{13} \mathrm{CO}^{+} / \mathrm{HN}^{13} \mathrm{C}$ and $\mathrm{HNCO} / \mathrm{C}^{18} \mathrm{O}$ ratios, respectively).

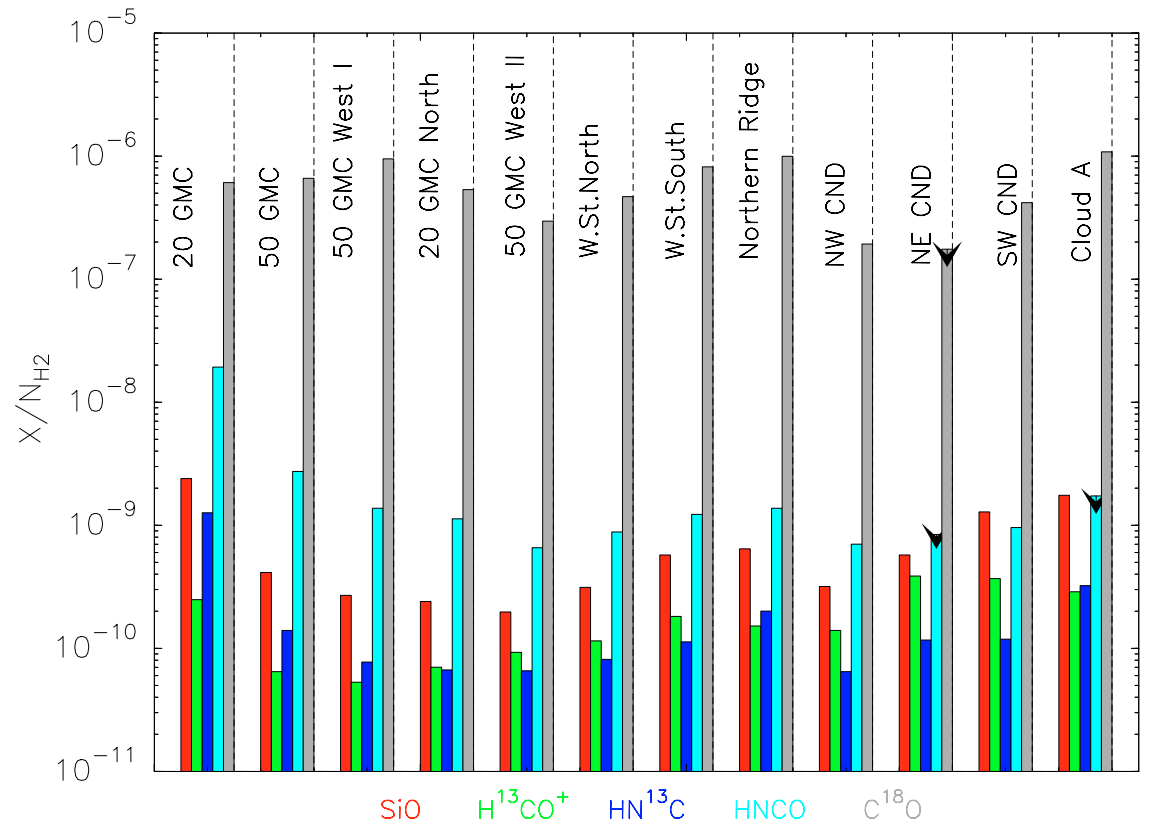

Fig. 8. Histogram with the fractional abundances shown in Table 4. $X$ stands for $\mathrm{SiO}$ (red), $\mathrm{H}^{13} \mathrm{CO}^{+}$(green), $\mathrm{HN}{ }^{13} \mathrm{C}$ (blue), $\mathrm{HNCO}$ (cyan), and $\mathrm{C}^{18} \mathrm{O}$ (gray), from left to right for every source. 

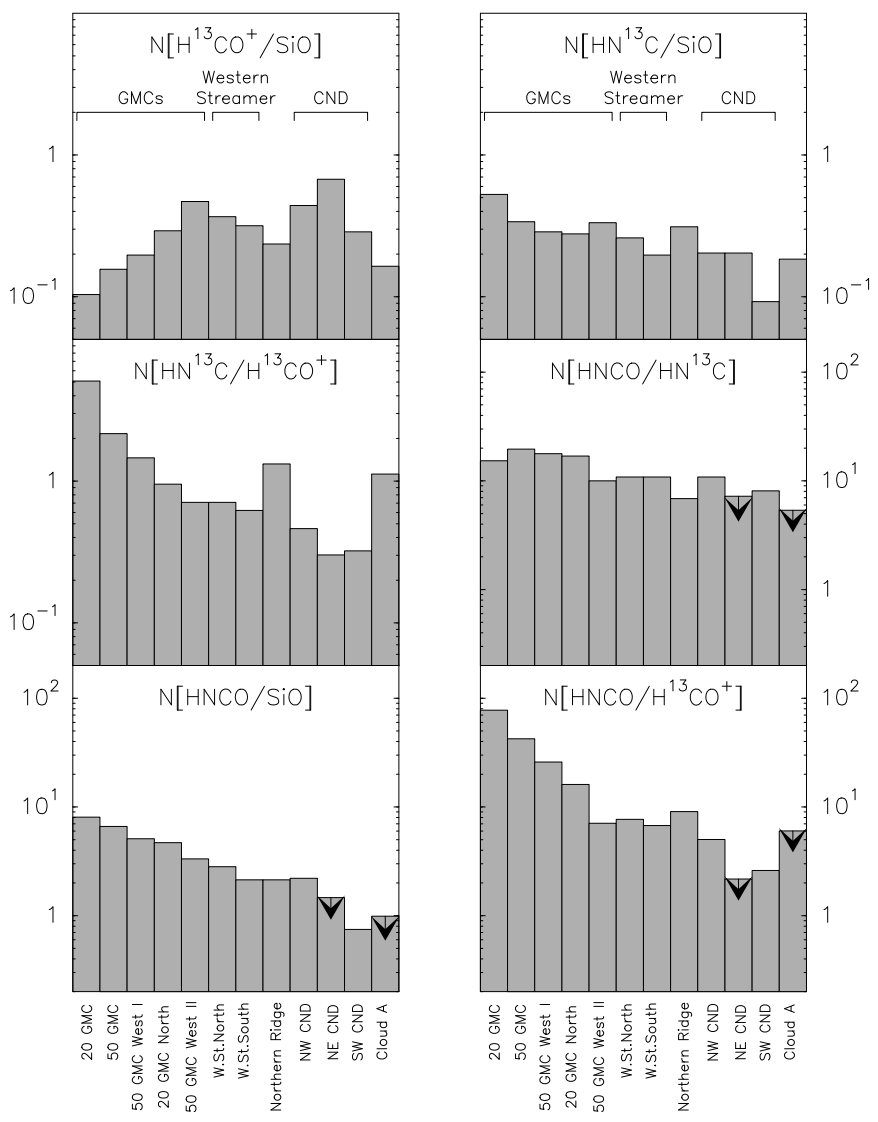

Fig. 9. Histogram with ratios between column densities shown in Table 3.

a smaller variation of a factor of 12 , and does not follow the trend observed in $\mathrm{HNCO}$. $\mathrm{SiO}$ fractional abundance is high at the cores of the GMCs, as well as in the positions of the CND. A similar behavior to $\mathrm{HNCO}$ follows $\mathrm{HN}^{13} \mathrm{C}$ (a factor of 20). $\mathrm{HN}^{13} \mathrm{C}$ seems to be anticorrelated with $\mathrm{H}^{13} \mathrm{CO}^{+}$, which shows the smallest variation in its fractional abundance after $\mathrm{C}^{18} \mathrm{O}$ (a factor of 7).

The same trends can be seen in the integrated intensity ratio maps. The highest $\mathrm{SiO}(2-1) / \mathrm{CS}(1-0)$ ratios and $\mathrm{SiO}$ fractional abundances are reached at the southwest lobe of the CND and Cloud A, only comparable to the ratio found at the GMCs. The gas components associated with the whole CND, the Western Streamer, and the Northern Ridge also present higher ratios than the surrounding gas (see Fig. B.1). The $\mathrm{H}^{13} \mathrm{CO}^{+}(1-0) / \mathrm{CS}(1-0)$ ratio seems to follow similar behavior to the $\mathrm{SiO}(2-1) / \mathrm{CS}(1-0)$ ratio, except for the gas components associated with the GMCs. The $\mathrm{HN}^{13} \mathrm{C}(1-0) / \mathrm{CS}(1-0)$ and $\mathrm{HNCO}(5-4) / \mathrm{CS}(1-0)$ ratios shares a similar behavior, reaching the maximum values of their ratios and abundances at the $20 \mathrm{~km} \mathrm{~s}^{-1} \mathrm{GMC}$, and decreasing toward the nuclear region.

Figure 9 presents histograms with the ratios between column densities of $\mathrm{SiO}, \mathrm{H}^{13} \mathrm{CO}^{+}, \mathrm{HN}^{13} \mathrm{C}$, and $\mathrm{HNCO}$ toward selected positions. Sources have been ordered from left to right according to the $\mathrm{HNCO} / \mathrm{SiO}$ column density ratio. There seems to be a relation between the $\mathrm{HNCO} / \mathrm{SiO}$ ratio and the distance to the nuclear region. All sources related with the GMCs are grouped with the highest ratios, the Western Streamer and Northern Ridge present intermediate values, whereas ratios toward the CND and Cloud A show the lowest values. Similar trends present all the other ratios, except for the $\mathrm{H}^{13} \mathrm{CO}^{+} / \mathrm{SiO}$ column density ratio. The $\mathrm{HNCO} / \mathrm{HN}^{13} \mathrm{C}$ ratio is rather uniform with only a factor of 4 difference between the maximum and minimum values. Variations in the $\mathrm{HN}^{13} \mathrm{C} / \mathrm{SiO}, \mathrm{H}^{13} \mathrm{CO}^{+} / \mathrm{SiO}$, and $\mathrm{HNCO} / \mathrm{SiO}$ ratios are within factors $6-8$, respectively, whereas variations in the $\mathrm{HN}^{13} \mathrm{C} / \mathrm{H}^{13} \mathrm{CO}^{+}$and $\mathrm{HNCO} / \mathrm{H}^{13} \mathrm{CO}^{+}$ratios are the greatest, with values of 17 and 40, respectively.

In summary, we can group the observed species in those whose abundances decrease ( $\mathrm{HNCO}$ and $\mathrm{HN}^{13} \mathrm{C}$ ) and those whose abundances are constant or enhanced $\left(\mathrm{SiO}\right.$ and $\left.\mathrm{H}^{13} \mathrm{CO}^{+}\right)$ toward the nuclear region.

\section{Discussion}

In this section, we discuss the most outstanding chemical differences found in our data in the context of the two main mechanisms considered to be the main drivers of the chemistry and the heating of the central region: shocks and UV radiation (see Sect. 1). To establish the dominant chemistry for the GC clouds from the observational point of view, we have selected several sources that are well-known prototypes of the different kinds of chemistry in the Milky Way and in nearby galaxies (see Table 5). A brief description of the physical conditions of these prototypical sources is given before the discussion. To avoid uncertainties associated with the different ways in which the fractional abundances were calculated for the prototypical sources, we do not compare fractional abundances, but rather their ratios (see Table 6). From all the sources in the central $12 \mathrm{pc}$ where we have derived abundances, we have selected the SW CND and 20 GMC as the most representative positions for the chemistry in this region. We find a difference of one order of magnitude between the $\mathrm{HNCO} / \mathrm{CS}$ and $\mathrm{HN}^{13} \mathrm{C} / \mathrm{CS}$ abundance ratios toward both sources, whereas the $\mathrm{SiO} / \mathrm{CS}$ and $\mathrm{H}^{13} \mathrm{CO}^{+} / \mathrm{CS}$ ratios show very similar values.

Finally, once we had defined the dominant chemistry drivers for each particular species, we used the effects that UV radiation produces on the molecular gas to establish the location of the different molecular components relative to the Central cluster.

\subsection{Prototypical sources}

As Galactic prototypical sources, we selected a cold dark cloud, a photo-dissociated region (PDR), molecular clouds interacting with an SNR and molecular outflows, and two hot cores.

TMC-1 is one of the best-studied quiescent dark clouds, with temperatures around $10 \mathrm{~K}$ and densities of a few $10^{4} \mathrm{~cm}^{-3}$. Due to the lack of internal heating sources and shocks, dark clouds are the best places to study gas-phase chemistry driven by ion-neutral reactions.

As PDR, we have selected the OMC-1 ridge. The UV radiation is provided mostly by the Trapezium stars, which are located at $0.25 \mathrm{pc}$ from the PDR. The mean kinetic temperature in the molecular layers of this PDR is around $85 \mathrm{~K}$ with $90 \%$ of the molecular gas in a homogeneous layer of density of $10^{4} \mathrm{~cm} \mathrm{~s}^{-1}$ and the remaining material concentrated in high-density clumps of $\sim 10^{6} \mathrm{~cm} \mathrm{~s}^{-1}$ (Hogerheijde et al. 1995). The UV radiation flux in the Orion PDR seems to be quite similar to that of the inner edge of the CND, with $G_{0} \sim 10^{5}$ (in units of the Habing field; Burton et al. 1990).

To test shock chemistry, we use clump G in the SNR IC 443, where shocks occur nearly perpendicular to the LOS. Densities and temperatures of the shocked gas are $10^{5}-3 \times 10^{6} \mathrm{~cm}^{-3}$ and 80-200 K, respectively (van Dishoeck et al. 1993). Unfortunately, HNCO has not been studied toward molecular clouds affected by SNRs. However, HNCO among other 
A\&A 526, A54 (2011)

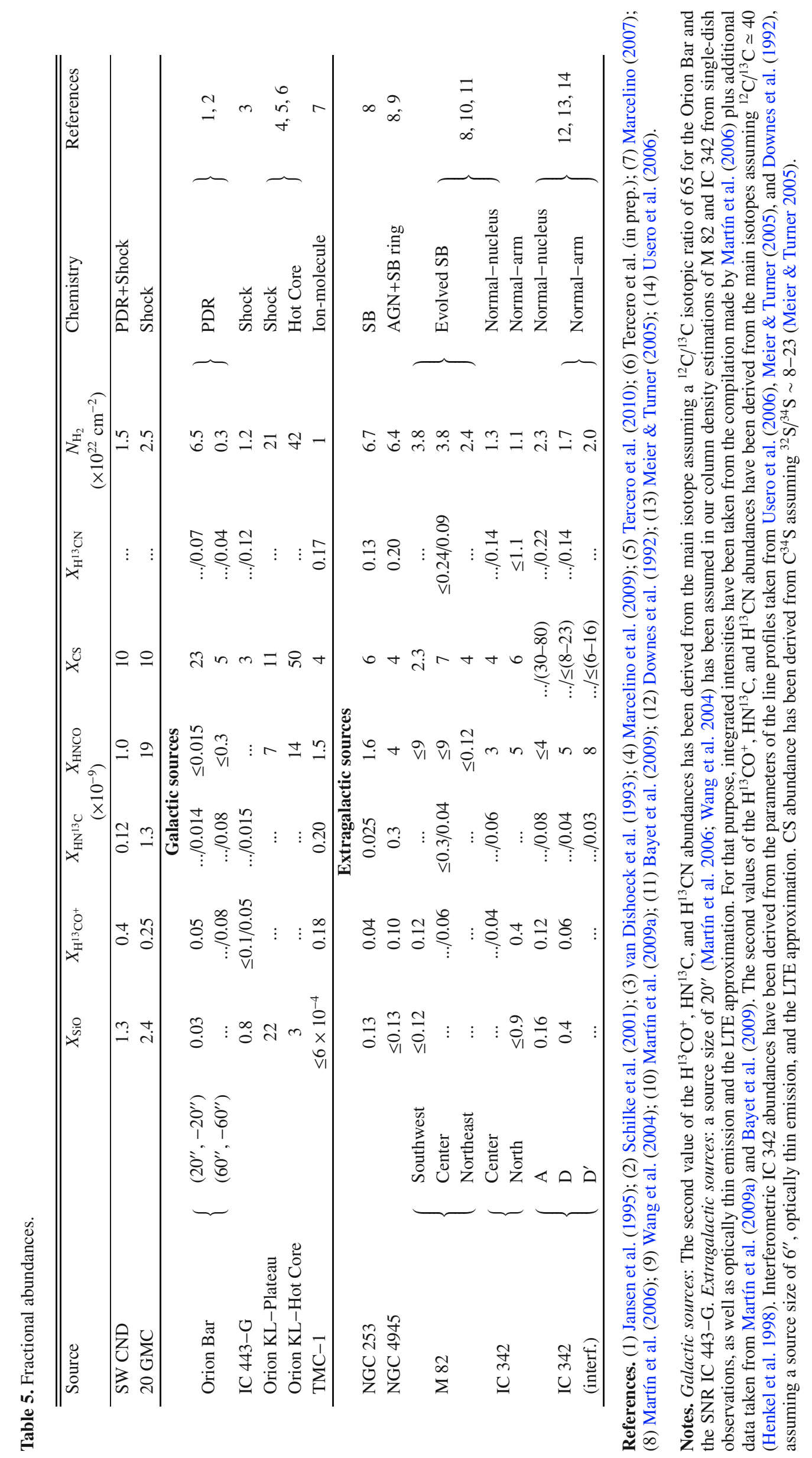


M. A. Amo-Baladrón et al.: Mapping the surrounding of Sgr A*

Table 6. Abundance ratios compared with prototypical Galactic and extragalactic sources.

\begin{tabular}{|c|c|c|c|c|c|c|}
\hline Source & & $\mathrm{SiO} / \mathrm{CS}$ & $\mathrm{H}^{13} \mathrm{CO}^{+} / \mathrm{CS}$ & $\mathrm{HN}^{13} \mathrm{C} / \mathrm{CS}$ & $\mathrm{HNCO} / \mathrm{CS}$ & $\mathrm{HN}^{13} \mathrm{C} / \mathrm{H}^{13} \mathrm{CN}$ \\
\hline SW CND & & 0.13 & 0.04 & 0.012 & 0.10 & $\ldots$ \\
\hline $20 \mathrm{GMC}$ & & 0.24 & 0.025 & 0.13 & 1.9 & $\ldots$ \\
\hline \multirow{7}{*}{$\begin{array}{l}\text { Orion Bar } \\
\text { IC } 443-G \\
\text { Orion KL-Plateau } \\
\text { Orion KL-Hot Core } \\
\text { TMC-1 }\end{array}$} & \multirow{7}{*}{$\begin{array}{l}\left(20^{\prime \prime},-20^{\prime \prime}\right) \\
\left(60^{\prime \prime},-60^{\prime \prime}\right)\end{array}$} & \multicolumn{4}{|c|}{ Galactic sources } & \multirow{4}{*}{$\begin{array}{c}\ldots / 0.20 \\
\ldots / 1.9 \\
\ldots / 0.13\end{array}$} \\
\hline & & $1.2 \times 10^{-3}$ & $2.0 \times 10^{-3}$ & $\ldots / 6 \times 10^{-4}$ & $\leq 7 \times 10^{-4}$ & \\
\hline & & & $\ldots / 0.015$ & $\ldots / 0.015$ & $\leq 0.07$ & \\
\hline & & 0.3 & $\leq 0.03 / 0.015$ & $\ldots / 5 \times 10^{-3}$ & $\ldots$ & \\
\hline & & 2.0 & $\ldots$ & $\ldots$ & 0.6 & $\ldots$ \\
\hline & & 0.05 & $\ldots$ & $\ldots$ & 0.3 & $\ldots$ \\
\hline & & $\leq 1.5 \times 10^{-4}$ & 0.04 & 0.05 & 0.4 & 1.2 \\
\hline & & \multicolumn{4}{|c|}{ Extragalactic sources } & \\
\hline NGC 253 & & 0.020 & $6 \times 10^{-3}$ & $4 \times 10^{-3}$ & 0.25 & 0.20 \\
\hline \multirow{2}{*}{ NGC 4945} & & $\leq 0.03$ & 0.025 & 0.08 & 1.0 & 1.6 \\
\hline & Southwest & $\leq 0.05$ & 0.05 & $\ldots$ & $\leq 4$ & $\ldots$ \\
\hline \multirow[t]{2}{*}{ M 82} & Center & $\ldots$ & $\ldots / 9 \times 10^{-3}$ & $\leq 0.04 / 6 \times 10^{-3}$ & $\leq 1.2$ & .../0.4 \\
\hline & Northeast & $\ldots$ & $\ldots$ & $\ldots$ & $\leq 0.03$ & $\ldots$ \\
\hline \multirow{3}{*}{ IC 342} & Center & & $\ldots / 0.010$ & $\ldots / 0.014$ & 0.8 & $\ldots / 0.4$ \\
\hline & North & $\leq 0.16$ & 0.07 & $\ldots$ & 1.0 & $\ldots$ \\
\hline & A & $(2.3-7) \times 10^{-3}$ & $(1.7-5) \times 10^{-3}$ & $\ldots /(1.1-3) \times 10^{-3}$ & $\leq 0.14$ & $\ldots / 0.23$ \\
\hline IC 342 & $\mathrm{D}$ & $\geq 0.018$ & $\geq 3 \times 10^{-3}$ & $\ldots / \geq 1.9 \times 10^{-3}$ & $\geq 0.25$ & $\ldots / 0.18$ \\
\hline (interf.) & $\mathrm{D}^{\prime}$ & $\ldots$ & $\ldots$ & $\ldots / \geq 1.8 \times 10^{-3}$ & $\geq 0.5$ & $\ldots$ \\
\hline
\end{tabular}

Notes. When two values for the abundance ratio are presented, the first one has been derived from the ${ }^{13} \mathrm{C}$ isotopic species and the second one from the main isotope assuming ${ }^{12} \mathrm{C} /{ }^{13} \mathrm{C} \approx 65$ for IC $443-\mathrm{G}$ and the Orion Bar (van Dishoeck et al. 1993; Jansen et al. 1995), and ${ }^{12} \mathrm{C} /{ }^{13} \mathrm{C} \simeq 40$ (Henkel et al. 1998) for the extragalactic sources. Interferometric column density ratios of IC 342 have been derived from $\mathrm{C}^{34} \mathrm{~S}$ assuming a range of ${ }^{32} \mathrm{~S} /{ }^{34} \mathrm{~S}$ isotopic ratios of $8-23$ (see Meier \& Turner 2005).

species, have been observed toward the closest high-mass starforming region in our Galaxy, the Orion KL cloud (Tercero et al. 2010). Single-dish line surveys find several spectral components within a telescope beam. One of these components, known as the plateau, presents a chemistry dominated by shocks driven by molecular outflows. The physical conditions of this component is characterized by temperatures and densities around $100-150 \mathrm{~K}$ and $\sim 10^{5} \mathrm{~cm}^{-3}$, respectively (see the introduction of Persson et al. 2007).

As prototypical hot cores, Sgr B2(N) and the hot core component of Orion KL were selected. The first one is the most prominent hot core of the Sgr B2 GMC complex located at the GC. It presents densities as high as $10^{7} \mathrm{~cm}^{-3}$ and temperatures that go from $20 \mathrm{~K}$ to up to $80 \mathrm{~K}$ averaged over a region of $1 \mathrm{pc}$, being even higher $(150 \mathrm{~K})$ on smaller scales (see Nummelin et al. 2000). The Orion hot core is a warm starforming region internally heated by one or more young massive protostars. It contains very dense clumps $\left(\sim 10^{7} \mathrm{~cm}^{-3}\right)$ and presents temperatures between $165-400 \mathrm{~K}$ (see the introduction of Persson et al. 2007).

On the other hand, as extragalactic prototypical sources, we selected three starburst nuclei in different evolutionary stages (NGC 253, M 82, and NGC 4945), and a normal spiral galaxy more similar to the Milky Way (IC 342). When one compares GC abundances with those of extragalactic nuclei measured with single-dish telescopes, it has to be taken into account that singledish measurements represent average values over regions of hundreds of parsecs that contains a large number of GMCs. At best, interferometer observations are able to provide average abundances over individual GMCs $(\sim 50 \mathrm{pc})$.

\subsection{SiO chemistry}

$\mathrm{SiO}$ is one of the best tracers of energetic mechanical phenomena in the ISM, with fractional abundances that changes by
5 orders of magnitude in dark clouds $\sim 10^{-12}$ to regions associated with shocks $\sim 10^{-7}$ (Martín-Pintado et al. 1992; Jiménez-Serra et al. 2005). Indeed, the most outstanding difference between TMC-1 and the central 12-pc sources is the $\mathrm{SiO} / \mathrm{CS}$ abundance ratio, since it is more than $2-3$ orders of magnitude larger in the SW CND and the 20 GMC than in cold clouds. The reason for this huge variation is the depletion of $\mathrm{SiO}$ onto grains in quiescent clouds. Only when energetic mechanisms destroy or erode dust grains does $\mathrm{SiO}$ appear with significant abundance in the gas phase.

The widespread $\mathrm{SiO}$ emission in the GC, with typical abundances of $\sim 10^{-9}$ (Martín-Pintado et al. 1997), has led to the conclusion that large-scale shocks must be at work in the GC. However, there are other energetic mechanisms, apart from shocks, that might enhance $\mathrm{SiO}$ abundances. It has been proposed that high-energetic phenomena, like X-rays as observed by the $6.4 \mathrm{keV}$ Fe line, enhance the $\mathrm{SiO}$ abundance (Martín-Pintado et al. 2000; Amo-Baladrón et al. 2009) in the GC over the averaged value. But, the 20 and $50 \mathrm{~km} \mathrm{~s}^{-1}$ GMCs do not seem to present an enhancement of the $6.4 \mathrm{keV} F e$ line, which would indicate a recent interaction of molecular gas with X-rays.

Another energetic mechanism that could enhance $\mathrm{SiO}$ abundance in PDRs is the desorption of grain mantles by UV-photons, as Schilke et al. (2001) have shown for the Orion Bar and the S140 PDR. However, the SiO/CS abundance ratio in the Orion Bar is 2 orders of magnitude lower than toward the SW CND and the 20 GMC. Thus, UV photo-desorption does not seem to play an important role in the enhancement of $\mathrm{SiO}$ in the central 12-pc of the GC.

On the other hand, the $\mathrm{SiO} / \mathrm{CS}$ abundance ratio in IC 443-G is very similar to those found toward the SW CND and the 20 GMC sources. However, the plateau component of Orion KL presents a $\mathrm{SiO} / \mathrm{CS}$ abundance ratio that is one order of magnitude greater than those found at our sources. According to 
the C-shock models of Jiménez-Serra et al. (2008), our observed $\mathrm{SiO}$ abundances $\left(\sim 10^{-9}\right)$ can be accounted for by the sputtering of grain mantles in shocks with velocities between 20 and $30 \mathrm{~km} \mathrm{~s}^{-1}$ (assuming that a significant amount of silicon is locked in the mantles). At higher shock velocities, sputtering of grain cores begins and gives rise to $\mathrm{SiO}$ abundances much higher $\left(\sim 10^{-7}-10^{-6}\right)$ than the ones derived toward the 12 central parsecs of the Galaxy.

Therefore, neither X-rays nor photo-desorption due to the UV field from the Central cluster can explain the $\mathrm{SiO}$ abundance in the central 12-pc sources, with C-shocks with velocities between $20-30 \mathrm{~km} \mathrm{~s}^{-1}$ the most likely origin of its abundance.

\subsection{HNCO chemistry}

Isocyanic acid (HNCO) has been observed in a wide variety of sources, from dark clouds to hot cores (see the introduction of Tideswell et al. 2010, for a brief review) with abundances of $\sim 10^{-9}-10^{-8}$. In the GC, HNCO emission has also been found to be particularly strong and widespread (Lindqvist et al. 1995; Kuan \& Snyder 1996; Dahmen et al. 1997; Sato et al. 2002; Minh \& Irvine 2006; Martín et al. 2008).

The $\mathrm{HNCO} / \mathrm{CS}$ abundance ratio in the $\left(20^{\prime \prime},-20^{\prime \prime}\right)$ position of the Orion Bar is 2-3 orders of magnitude lower than in the central 12-pc sources. Moreover, among all the sources listed in Table 5, the Orion Bar is where one can find the lowest HNCO abundances. The behavior of HNCO in PDRs is completely opposite that of CS. Martín et al. (2008) have studied the HNCO emission toward several positions in the GC, finding that the $\mathrm{HNCO}$ abundance decreases by up to a factor 22 for the sources affected by photodissociation, with maximum values of $2 \times 10^{-8}$ toward the cores of GMCs and minimum values of $1 \times 10^{-9}$ toward the sources close to massive stellar clusters. Moreover, this difference factor increases up to 30 when the $\mathrm{HNCO} / \mathrm{CS}$ abundance ratio is compared. The HNCO abundance toward the SW CND is one order of magnitude lower than toward the $20 \mathrm{GMC}$, which is consistent with the idea that HNCO is very easily photodissociated by UV radiation, as the SW CND is closer to the Central cluster and/or not as well-shielded as the 20 GMC.

Martín et al. (2009b) have modeled the HNCO abundance in PDRs as a function of the visual extinction with $T_{\mathrm{K}}=50 \mathrm{~K}, n_{\mathrm{H}_{2}}=10^{5} \mathrm{~cm}^{-3}$, and a UV radiation field of $G_{0}=5 \times 10^{3}$ in Habing units, similar to the physical conditions of galactic nuclei. In their coupled dense core-PDR model, they find observable HNCO abundances only for $A_{\mathrm{v}} \geq 5$ (i.e. $N_{\mathrm{H}_{2}} \sim 5 \times 10^{21} \mathrm{~cm}^{-2}$ ), concluding that molecular clouds affected by UV radiation with observable HNCO abundances must contain well shielded dense cores where HNCO molecules are protected against UV photons. According to Martín et al. (2009b), this could be the reason the HNCO/CS ratio show a wide range of values toward extragalactic nuclei, from the upper limit of 0.03 in M 82-NE to the high values $(0.8-1.0)$ found toward IC 342 and NGC 4945, with the ratio in NGC 253 in between $(0.25$; see Table 6$)$. The low ratio measured toward the GMCs of M 82 is the result of large amounts of gas being affected by UV radiation in its nuclear region. This is due to the lower column densities that M 82 GMCs present in comparison with GMCs of other extragalactic nuclei, where higher HNCO/CS ratios are found (Martín et al. 2009b).

Therefore, our low measured value for the HNCO/CS abundance ratio toward the SW CND reflects the effect of UV radiation on the fragile HNCO molecules. Column densities measured in the 12 central parsecs are $\sim 10^{22} \mathrm{~cm}^{-2}$, with the lowest values found toward Cloud $\mathrm{A}$ and the NE CND, just the sources where no emission of HNCO has been detected.

In contrast to the destruction mechanism of HNCO, the origin of this species is not yet well understood. Gas-phase chemistry alone is not able to reproduce the observed HNCO abundances, predicting abundances on the order of $10^{-10}$ (Iglesias 1977). Chemistry in post-shocked gas driven by neutral-neutral reactions has been invoked to reproduce HNCO abundances on the order of $10^{-8}$, as the high temperatures achieved by shocks could overcome the energy barriers of these reactions (for more details see Turner et al. 1999; Tideswell et al. 2010; Rodriguez-Fernandez et al. 2010). However, this formation pathway presents two main drawbacks: the short times that shocks can maintain the required high temperatures and cannot detect $\mathrm{O}_{2}$ in the $\mathrm{Sgr}$ A complex $\left(X_{\mathrm{O}_{2}} \leq 1.2 \times 10^{-7}\right.$; Sandqvist et al. 2008), a key molecule in this formation path of HNCO.

The alternative to HNCO production in gas phase is formation through grain-surface chemistry. Hot core chemical models (Garrod et al. 2008; Tideswell et al. 2010), as well as gasgrain chemistry models in cold clouds (Hasegawa \& Herbst 1993), generate high abundances of HNCO in the grain mantles $\left(10^{-7}-10^{-5}\right)$. There are also observational clues that point to a grain-surface origin for HNCO: the "XCN" infrared (IR) absorption feature of interstellar ices that could correspond to the $\mathrm{OCN}^{-}$anion (Hudson et al. 2001). Another observational proof is the correlation between the HNCO and SiO-integrated and peak intensities found by Zinchenko et al. (2000), with $\mathrm{SiO}$ lines broader than HNCO lines, which suggests a common origin. Besides, HNCO abundances are also enhanced in high-velocity gas (Zinchenko et al. 2000). Moreover, the largest $\mathrm{SiO} / \mathrm{CS}$ and $\mathrm{HNCO} / \mathrm{CS}$ abundance ratios among our selected prototypical Galactic sources are found at the plateau component of the Orion KL cloud, a high-mass star-forming region affected by several outflows.

However, after the formation of HNCO on the grain mantles, according to hot core chemical models, HNCO is destroyed by reactions with radicals originating more complex species, such as $\mathrm{NH}_{2} \mathrm{CHO}$. Under this scenario, the observed HNCO abundances in the gas-phase would be the result of the destruction of these more complex molecules (Garrod et al. 2008; Tideswell et al. 2010). Ejection of more complex molecules with the subsequent photodissociation and production of HNCO in gas-phase also seems unlikely in the GC sources, as we observed the highest HNCO abundances toward the more shielded cores of the GMCs and the lowest ones toward regions with strong UV fields that should favour the destruction of the complex molecules. Moreover, observational results seem to contradict the idea of HNCO as a second generation species. Bisschop et al. (2007) have measured a set of complex organic molecules toward a sample of hot cores. $\mathrm{HNCO}$ and $\mathrm{NH}_{2} \mathrm{CHO}$ belong to the same group, that is, they are evaporated from grain mantles and are very likely first generation species (i.e. directly released from the grains), as their abundances toward several sources are correlated.

Therefore, HNCO could share the same origin as $\mathrm{SiO}$, shocks, but HNCO seems to be more sensitive to UV radiation. IC 342, a similar nearby galaxy to the Milky Way, has been mapped with interferometry technique in several species, revealing an spiral galaxy composed of several GMCs with chemistries driven by different mechanisms. The $\mathrm{SiO} / \mathrm{CS}$ and $\mathrm{HNCO} / \mathrm{CS}$ ratios toward the GMC closer to the stellar cluster (GMC A) in IC 342 are lower than those found toward the GMCs in the spiral arms (GMCs D and $\left.\mathrm{D}^{\prime}\right)$. The same trend is followed by our SW CND and 20 GMC sources, with the upper limit to 
the $\mathrm{HNCO} / \mathrm{CS}$ abundance ratio at the GMC A of IC 342 similar to our derived value at the SW CND source. The GMC A could be suffering the direct impact of the UV radiation, stellar winds, and SN explosions originated in the central cluster (Schinnerer et al. 2008). Therefore, both sources seem to be affected by strong UV radiation and shocks. In contrast, the GMCs in the spiral arms of IC 342 show higher lower limits to the $\mathrm{SiO} / \mathrm{CS}$ and $\mathrm{HNCO} / \mathrm{CS}$ ratios, which could approach the 20 GMC ratios. This is consistent with the idea that the GMCs in the spiral arms are affected by shocks, but not strongly by UV radiation. Meier \& Turner (2005) also mapped methanol $\left(\mathrm{CH}_{3} \mathrm{OH}\right)$, finding abundance enhancements that can be produced by large-scale mild shocks due to orbital dynamics, as $\mathrm{CH}_{3} \mathrm{OH}$ cannot be ejected to the gas by evaporation and the emission of both species is widespread. Thus, interferometric observations of IC 342 seem to support the idea of grain chemistry as the origin of HNCO.

\section{4. $\mathrm{H}^{13} \mathrm{CO}^{+}$chemistry}

The $\mathrm{H}^{13} \mathrm{CO}^{+} / \mathrm{CS}$ abundance ratio toward the Orion PDR presents a difference of almost an order of magnitude between the offset positions $\left(20^{\prime \prime},-20^{\prime \prime}\right)$ and $\left(60^{\prime \prime},-60^{\prime \prime}\right)$ with respect to the ionization front (IF; see Table 6; Jansen et al. 1995). This difference is due to a larger increase in the CS abundance toward the closest position to the IF, which is $\left(20^{\prime \prime},-20^{\prime \prime}\right)$, whereas the $\mathrm{HCO}^{+}$abundance mostly remains constant. PDR-models show that higher $\mathrm{HCO}^{+} / \mathrm{CS}$ abundance ratios are expected toward the superficial layers of PDRs than toward cool cores (Sternberg \& Dalgarno 1995). The exception to this trend could be the most UV-exposed layer of PDRs. There, ion destruction is very efficient through dissociative recombination reactions, as electron densities reach their maximum values. In the Horsehead edgeon PDR, the HCO emission traces the edge of the PDR, whereas the $\mathrm{H}^{13} \mathrm{CO}^{+}$emission appears more shifted to the cloud interior (Goicoechea et al. 2009).

Our $\mathrm{H}^{13} \mathrm{CO}^{+} / \mathrm{CS}$ abundance ratios are similar to that of the $\left(60^{\prime \prime},-60^{\prime \prime}\right)$ position of the Orion Bar, although the SW CND ratio is slightly larger (by factors of 2-2.6) and more similar to the value found at the dark cloud TMC-1, despite the strong UV field that impinges on the CND. This could be due to the different extension sampled by the telescope beam in the Jansen et al. survey $(0.04 \mathrm{pc})$ and our work $(1.2 \mathrm{pc})$. Our beam samples not only the PDR, but also a more shielded part of the cloud. Thus, the $\mathrm{H}^{13} \mathrm{CO}^{+} / \mathrm{CS}$ abundance ratio could reflect this mixture, approaching the value found in dark clouds.

Conversely to the $\mathrm{HCO}^{+}$chemistry in PDRs and dark clouds, there is no agreement with a possible $\mathrm{HCO}^{+}$enhancement in shocks. Studies of molecular outflows (Garden \& Carlstrom 1992; Girart et al. 1999) have found enhancements of the $\mathrm{HCO}^{+}$ abundance in shocked gas. $\mathrm{HCO}^{+}$abundance enhancement of one order of magnitude have been detected in the interface between high-velocity outflows and the surrounding quiescent gas in low-mass star-forming regions (Hogerheijde et al. 1998). This enrichment would be produced by the sputtering of $\mathrm{CO}$ and $\mathrm{H}_{2} \mathrm{O}$ to gas phase from ice mantles, followed by photochemical processing by $\mathrm{UV}$ radiation, generating $\mathrm{C}^{+}$that would react with $\mathrm{H}_{2} \mathrm{O}$ to enhance the $\mathrm{HCO}^{+}$abundance (Rawlings et al. 2000; Viti et al. 2002). However, there are also chemical models and observational results that report a decrease in the $\mathrm{HCO}^{+}$abundance in the postshock gas with respect to the preshock gas (see van Dishoeck et al. 1993; Garay et al. 1998, and references therein).
Our SW CND and 20 GMC sources present values of the $\mathrm{H}^{13} \mathrm{CO}^{+} / \mathrm{CS}$ abundance ratio in between those of TMC-1, clump $\mathrm{G}$, and the $\left(60^{\prime \prime},-60^{\prime \prime}\right)$ position of the Orion Bar. The highest value of this ratio is the one of TMC-1, a prototype of quiescent cloud. This suggests that the ion abundance in our sources is not increased by shocks, despite the favorable conditions that this region presents, i.e., shocks plus UV radiation.

\subsubsection{The GC ionization rate}

Oka et al. (2005) claim that the GC presents a higher ionization rate than anywhere else in the Galaxy $\left(\zeta=2-7 \times 10^{-15} \mathrm{~s}^{-1}\right)$. $\mathrm{H}_{3}^{+}$absorption lines toward selected stars reveal the presence of extensive hot and diffuse clouds with high-velocity dispersions in the inner $200 \mathrm{pc}$ of the Galaxy. Oka et al. (2005) found broad absorptions due to $\mathrm{H}_{3}^{+}(3,3)$ in the direction of the $50 \mathrm{~km} \mathrm{~s}^{-1} \mathrm{GMC}$, consistent with its velocity range. However, Geballe et al. (1989), from CO in absorption, prefer to establish the absorption origin at the inner edge of the CND.

Farquhar et al. (1994) have studied the effects of varying the cosmic-ray ionization rate in dark clouds. Their model predicts an increase in the $\mathrm{HCO}^{+}$abundance as ionization rate becomes higher. Our $\mathrm{HCO}^{+}$abundances, derived taking a ${ }^{12} \mathrm{C} /{ }^{13} \mathrm{C}$ isotopic ratio of 20-25 (Wilson 1999) into account, are between $\sim 10^{-9}-10^{-8}$. The maximum value corresponds to the SW CND that, according to Farquhar et al.'s model, could be explained by the "standard" cosmic-ray ionization rate $\left(\zeta \sim 10^{-17} \mathrm{~s}^{-1}\right)$. On the other hand, the minimum value, corresponding to the 50 GMC, could be produced by an ionization rate a factor of $\sim 60$ lower than the "standard" value. Therefore, a small gradient in the ionization rate could be responsible for the variations found in the $\mathrm{H}^{13} \mathrm{CO}^{+}$abundances toward the CND and the 50 GMC. However, our values do not support the high ionization rate derived by $\mathrm{Oka}$ et al. (2005). When modeling of the observed intensities of $\mathrm{H}_{2} \mathrm{O}$ and $\mathrm{H}_{3} \mathrm{O}^{+}$toward the $\mathrm{Sgr} \mathrm{B} 2$ envelope, van der Tak et al. (2006) obtain a cosmic-ray ionization rate of $\sim 4 \times 10^{-16} \mathrm{~s}^{-1}$, lower than the value derived by Oka et al. (2005) in diffuse clouds. The physical conditions of the Sgr B2 envelope $\left(T_{\mathrm{k}}=60 \mathrm{~K}\right.$ and $\left.n_{\mathrm{H}_{2}}=10^{6} \mathrm{~cm}^{-3}\right)$ are more similar to those of the molecular gas traced by our observations.

\section{5. $H N^{13} \mathrm{C}$ chemistry}

The chemistry of HNC is usually studied simultaneously with that of $\mathrm{HCN}$. Unfortunately, we cannot compare $\mathrm{HN}^{13} \mathrm{C}$ with $\mathrm{H}^{13} \mathrm{CN}$ abundances in our sources. However, HCN and CS seem to share a constant abundance in regions dominated by different kinds of chemistries. Both species are not strongly affected by shocks or by UV radiation. Therefore, we expect that the $\mathrm{HN}^{13} \mathrm{C} / \mathrm{CS}$ and $\mathrm{HN}^{13} \mathrm{C} / \mathrm{H}^{13} \mathrm{CN}$ abundance ratios follow a similar trend. From Table 6, the lowest values of the $\mathrm{HN}^{13} \mathrm{C} / \mathrm{H}^{13} \mathrm{CN}$ abundance ratio are associated with the sources that present the lowest values of the $\mathrm{HN}^{13} \mathrm{C} / \mathrm{CS}$ abundance ratio, the $\left(20^{\prime \prime},-20^{\prime \prime}\right)$ position of the Orion Bar and IC 443-G. Conversely, the highest values of both ratios are found toward the $\left(60^{\prime \prime},-60^{\prime \prime}\right)$ position of the Orion PDR and the dark cloud TMC-1. Following this trend, one can predict a lower $\mathrm{HN}^{13} \mathrm{C} / \mathrm{H}^{13} \mathrm{CN}$ abundance ratio at the SW CND compared with the 20 GMC. This result agrees with previous studies that suggest that the HNC molecule is very sensitive to temperature, since it is depleted toward regions of high temperatures caused by shock or UV-heating. 
In quiescent cool dark clouds, the $\mathrm{HNC} / \mathrm{HCN}$ abundance ratio is close to or higher than unity $(2.1 \pm 1.2$ in a range of 0.54-4.5; Hirota et al. 1998). The HNC/HCN ratio decreases by 1-2 orders of magnitudes in the warmer GMCs near sites of massive star formation. Values ranging between $0.013-0.2$ have been derived toward selected positions in the GMC OMC-1, with the lowest ratios found in the immediate vicinity of the hot core Orion-KL (Schilke et al. 1992). These results suggest a correlation between the $\mathrm{HNC} / \mathrm{HCN}$ abundance ratio and temperature. Moreover, Hirota et al. (1998) claimed that this decrease in the ratio is due to destruction processes of $\mathrm{HNC}$ rather than to formation processes of $\mathrm{HCN}$, as the $\mathrm{HCN}$ abundances in OMC-1 are almost the same than in dark cloud cores, whereas the HNC abundances in OMC-1 are generally lower. According to gas-phase chemical models, $\mathrm{HCN}$ and $\mathrm{HNC}$ are mainly produced by dissociative recombination of $\mathrm{HCNH}^{+}$, leading to $\mathrm{HNC}$ on $60 \%$ and $\mathrm{HCN}$ on $40 \%$ of collisions, as observed abundances toward cold dark clouds suggest (Hirota et al. 1998). The dependence of HNC abundance on temperature has been explained by Pineau des Forets et al. (1990) as due to neutralneutral reactions with an activation barrier. Hirota et al. (1998) derived an activation barrier of $190 \mathrm{~K}$ for the destruction reactions of HNC with $\mathrm{H}$ and $\mathrm{O}$. However, this value could be higher according to quantum chemical calculations, making the destruction of $\mathrm{HNC}$ effective only in regions with temperatures higher than $300 \mathrm{~K}$ (Talbi et al. 1996).

As we can see in Table 3, the 20 GMC is the source that presents the lowest volume density of all our sources, with $n_{\mathrm{H}_{2}}=0.4 \times 10^{5} \mathrm{~cm}^{-3}$ at $T_{\mathrm{K}}=50 \mathrm{~K}$. As previously mentioned, a decrease in the temperature is compatible with an increase in the volume density. Thus, the 20 GMC could present a lower density or, alternatively, a lower temperature than the surrounding molecular gas, leading to less efficiency of the HNC destruction processes there.

\subsection{The chemistry of the central 12 parsecs of the GC}

In summary, from the comparison of the abundance ratios derived toward the 12 central parsecs of the GC with that of prototypical Galactic sources, one can clearly conclude that shocks are responsible for maintaining the high $\mathrm{SiO}$ abundances found at the CND and the GMCs, while the strong UV radiation also affects the dense gas of the CND. This strong UV field seems to be responsible for the low abundances of HNCO and $\mathrm{HN}^{13} \mathrm{C}$ measured toward the CND. Increasing temperatures due to UV-heating as one approaches the Central cluster could be responsible for the observed decrease in the $\mathrm{HN}^{13} \mathrm{C}$ abundance. The $\mathrm{HN}^{13} \mathrm{C}$ peak abundance is found toward the well-shielded 20 GMC, a molecular cloud that could be either less dense or colder than the surrounding molecular gas. As shocks are widespread all over the central region, it is not expected that they have produced the differences found in the $\mathrm{HN}^{13} \mathrm{C}$ abundances. High temperatures can also favor $\mathrm{HNCO}$ formation in gas-phase. However, our observed abundance ratios toward the CND indicate that HNCO abundance is strongly depleted there, as well as in Galactic PDRs. The high $\mathrm{SiO}$ and $\mathrm{HNCO}$ abundance ratios found toward the 20 and 50 GMCs can be explained by the presence of nondissociative C-shocks with velocities between 20 and $30 \mathrm{~km} \mathrm{~s}^{-1}$, able to eject $\mathrm{Si}$ or $\mathrm{SiO}$ from grain mantles into the gas phase. HNCO could also be locked in grain mantles and directly realeased to the gas phase by these $\mathrm{C}$-shocks. Once in the gas phase, unshielded HNCO molecules would be rapidly destroyed by the UV radiation, leading to the low HNCO abundances found at the CND molecular gas. The small gradient found in the $\mathrm{H}^{13} \mathrm{CO}^{+}$abundance from the CND to the $50 \mathrm{GMC}$ could be explained by a decrease of one order of magnitude in the ionization rate with respect to the "standard" value toward this GMC, which presents the highest column density of all our sources.

\subsection{The $\mathrm{HNCO}(5-4) / \mathrm{SiO}(2-1)$ ratio as a probe of the distance to the nuclear region}

The abundance ratios derived in Sect. 5 show a possible relation between the $\mathrm{HNCO} / \mathrm{SiO}$ ratio and the distance to the nuclear region. The $\mathrm{HN}^{13} \mathrm{C} / \mathrm{H}^{13} \mathrm{CO}^{+}$and $\mathrm{HNCO} / \mathrm{H}^{13} \mathrm{CO}^{+}$abundance ratios present a similar trend, with the most contrasted column density ratios. However, $\mathrm{H}^{13} \mathrm{CO}^{+}$suffers from absorption in the direction of $\mathrm{Sgr} \mathrm{A}$, whereas the $\mathrm{HN}^{13} \mathrm{C}$ emission is not in general very intense. On the other hand, $\mathrm{SiO}$ and $\mathrm{HNCO}$ present strong emission in the regions where they are detected. As we have previously discussed in Sects. 6.2 and 6.3, SiO and HNCO seem to share a common origin, that is, both molecules are locked in grain mantles and can be released to the gas phase by shocks. However, HNCO is photodissociated more efficiently than $\mathrm{SiO}$. In Sect. 5 we have also seen that excitation effects do not modify the general trends found in the integrated intensity ratio maps. Therefore, the $\mathrm{HNCO} / \mathrm{SiO}$ intensity ratio should provide information on the relative distance of the observed molecular gas to the Central cluster, the source of the UV radiation giving rise to the photodissociation in the nuclear region (Krabbe et al. 1995; Figer 2008).

The $\operatorname{HNCO}(5-4)$ emission not only decreases in the highvelocity gas, but also in the velocity components related to the GMCs. Figure 10 shows the $\operatorname{HNCO}(5-4) / \operatorname{SiO}(2-1)$, $\mathrm{SiO}(2-1) / \mathrm{CS}(1-0)$, and $\mathrm{HNCO}(5-4) / \mathrm{CS}(1-0)$ intensity ratios (hereafter the $\mathrm{HNCO} / \mathrm{SiO}, \mathrm{SiO} / \mathrm{CS}$, and $\mathrm{HNCO} / \mathrm{CS}$ ratios) for low, intermediate, and high-velocity gas ranges along four different directions. The Northern Ridge (velocity range between $\left.[-20,0] \mathrm{km} \mathrm{s}^{-1}\right)$, the Southern Streamer $([-60,0],[20,40]$, and $\left.[60,120] \mathrm{km} \mathrm{s}^{-1}\right)$, the $50 \mathrm{~km} \mathrm{~s}^{-1}$ Streamer $([-90,-50]$, $[20,60]$, and $\left.[60,100] \mathrm{km} \mathrm{s}^{-1}\right)$, and the Molecular Ridge $\left([20,60] \mathrm{km} \mathrm{s}^{-1}\right)$. Maps at the top of Fig. 10 indicate the spatially integrated regions where we derived the integrated instensity ratios. In the following, we discuss each of this streamers comparing our instensity ratios with the previous pictures of the $3 \mathrm{D}$ arrangement proposed in the literature, trying to place them along the LOS:

- The Molecular Ridge: the intensity ratio along these ridge is a good example of a rather constant $\mathrm{HNCO} / \mathrm{SiO}$ ratio with only differences of a factor of 1.5 between the maximum and minimum observed values. This suggests that there the molecular gas is not strongly affected by UV radiation due to its distance to the UV source and/or its shielding from the UV photons. The values of the $\mathrm{HNCO} / \mathrm{SiO}, \mathrm{SiO} / \mathrm{CS}$, and $\mathrm{HNCO} / \mathrm{CS}$ ratios are around $1.8,0.6$, and 0.6 , respectively.

- The Northern Ridge: this filamentary feature is located on the northern edge of Sgr A East, as can be seen from the $[-20,10] \mathrm{km} \mathrm{s}^{-1}$ map at the top-left of Fig. 10. This ridge shows low $\mathrm{HNCO} / \mathrm{SiO}$ ratios $(<0.7)$ along all its full length (even for the more distant pixels from the nucleus), with a systematic decrease (by factor of $\geq 2.5$ ) as we approach the center. This decrease must be due to the photodissociation of $\mathrm{HNCO}$, as the $\mathrm{SiO} / \mathrm{CS}$ ratio shows a constant value along its length $(\sim 0.4)$. The HNCO/CS ratio is low $(0.11-0.3)$ compared to that of the Molecular Ridge and the GMCs. It is likely that an even larger change in the $\mathrm{HNCO} / \mathrm{SiO}$ ratio could be achieved along this filament if the mapped area would have been extended further to the 
M. A. Amo-Baladrón et al.: Mapping the surrounding of Sgr A*
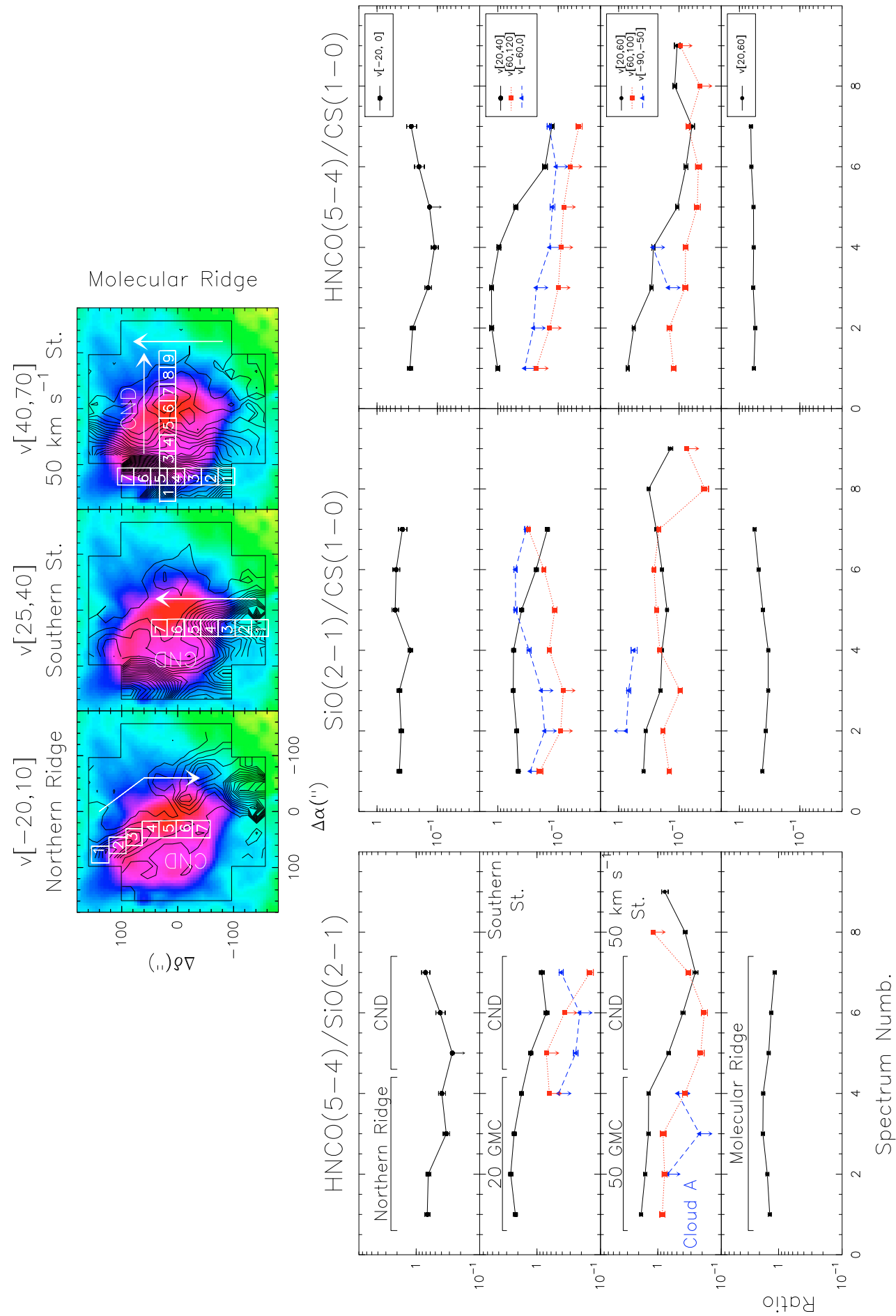

Fig. 10. Velocity-integrated $\mathrm{HNCO}(5-4) / \mathrm{SiO}(2-1)$ (left), $\mathrm{SiO}(2-1) / \mathrm{CS}(1-0)$ (middle), and $\mathrm{HNCO}(5-4) / \mathrm{CS}(1-0)$ (right) ratios along four different directions and different velocity intervals. The four different directions are indicated at the top of the left panels, and they are pointed out with white arrows on the three maps located at the top of the figure. These maps show, in contours, the $\mathrm{SiO}(2-1)$ emission in three different velocity ranges (indicated at the top of each map), superimposed on the radio continuum image of Yusef-Zadeh \& Morris (1987) at $20 \mathrm{~cm}$ on a color scale. The different velocity intervals in which we have integrated the molecular emission are indicated at the upper-right corner of the right panels. For example, three velocity-integrated ratios are shown along the Southern Streamer direction. The one corresponding to the velocity interval of this streamer, $[20,40] \mathrm{km} \mathrm{s}^{-1}$, is shown with black circles connected by black lines, whereas those corresponding to lower and higher velocity intervals, $[-60,0]$ and $[60,120] \mathrm{km} \mathrm{s}^{-1}$, are represented by blue triangles connected by dashed lines and red squares connected by dotted lines, respectively. The $x$-axis of the ratio plots represents the spectrum number, that increases from northeast to south in case of the Northern Ridge (1st plot beginning from the top), from south to north in the Southern Streamer (2nd), from east to west in the $50 \mathrm{~km} \mathrm{~s}^{-1} \mathrm{Streamer}$ (3rd), and from south to north in the Molecular Ridge (4th), i.e., in the direction of the white arrows. The white open squares, marked with the spectrum numbers, surround the region where the ratios have been derived, whose spectra can be seen in Fig. 11. Uncertainties in the ratio values represented by the errorbars have been calculated propagating the uncertainties in the intensities, which have been derived assuming a conservative $20 \%$ calibration error. 
north of the ridge, as this feature seems to be connected with the $-30 \mathrm{~km} \mathrm{~s}^{-1}$ molecular cloud (Serabyn \& Güsten 1987). Our results support the idea that this cloud could be feeding the CND through the Northern Ridge.

McGary et al. (2001) find a connection between this ridge and the northeastern region of the CND. These authors find a smooth positive velocity gradient in their $\mathrm{NH}_{3}$ maps from north to south (from $-10 \mathrm{~km} \mathrm{~s}^{-1}$ in the Northern Ridge to $60 \mathrm{~km} \mathrm{~s}^{-1}$ in the as a function northeastern region of the CND). In the case that interaction between the ridge and the CND occurs, this velocity gradient places the ridge in front of the northeastern part of the CND. A similar gradient can be observed in the declination-velocity plot of the $\mathrm{SiO}(2-1)$ emission averaged between RA $=$ [45", 105" $]$ in Fig. 3. McGary et al. (2001) also find an increase in the $\mathrm{NH}_{3}(2,2) / \mathrm{NH}_{3}(1,1)$ line ratios that suggests an increase in temperature where the streamer intersects the CND.

Dust emission is also observed from the ridge, but it disappears in the connexion region between the CND and the ridge, which McGary et al. (2001) interpret as destruction of dust by UV-photons from the Central cluster. However, Lee et al. (2008) suggest, from their kinematical comparison of shocked gas traced by $\mathrm{H}_{2}$ emission with quiescent gas traced by $\mathrm{NH}_{3}$ emission, that this feature is on the far side of the Sgr A East SNR, and is accelerated away from us due to the SNR expansion. Both scenarios would be irreconcilable. In the case that the nuclear region were inside or in the leading edge of Sgr A East and the Northern Ridge were behind the SNR, the Northern Ridge could not be connected with the CND. Karlsson et al. (2003) have carried out an interferometric mapping of the Sgr A complex in the four $18-\mathrm{cm} \mathrm{OH}$ absorption lines. The Northern Ridge is clearly seen in their 1665 and $1667 \mathrm{MHz}$ maps, providing a strong argument against the idea of Lee et al. (2008), suggesting that the ridge is in front of the Sgr A East SNR, i.e.: in front of the continuum emission. Our low $\mathrm{HNCO} / \mathrm{SiO}$ ratio implies the Northern Ridge must be close to the nucleus, which supports the interaction scenario.

- The Southern Streamer: this long filament extends northward from the $20 \mathrm{~km} \mathrm{~s}^{-1}$ GMC toward the southeastern edge of the CND. It has been observed in several transitions of $\mathrm{NH}_{3}$ (Okumura et al. 1991; Ho et al. 1991; Dent et al. 1993; Coil \& Ho 1999, 2000; McGary et al. 2001; Herrnstein \& Ho 2002, 2005), and in methanol (Szczepanski et al. 1991). The $\mathrm{HNCO} / \mathrm{SiO}$ ratio along the Southern Streamer shows the largest gradient with a variation of a factor of $\sim 18$ between the ratio in the GMC $(\sim 2.6$ in the intermediate-velocity range) and in the CND $(\sim 0.15$ in the high-velocity range). Furthermore, the $\mathrm{HNCO} / \mathrm{CS}$ ratio decreases by a factor of 28 , with extreme values of $\sim 1.3$ and $\sim 0.05$ in the GMC and the CND, respectively. This decrease in the ratio values as we move toward the center is also observed if we only consider the intermediate velocity range (a difference of a factor of 4 in the $\mathrm{HNCO} / \mathrm{SiO}$ ratios), which could indicate that this streamer is approaching the central region and, therefore, a possible connection with the CND. Similar to the Northern Ridge, this decrease in the ratio is probably caused by the photodissociation of the HNCO, together with the enhancement of the $\mathrm{SiO}$ abundance in the $\mathrm{CND}$, combined with a less effective photodissociation rate of HNCO in the GMC. The SiO/CS ratio decreases by a factor of 4 as we approach the center in the intermediate velocity range, whereas the $\mathrm{HNCO} / \mathrm{CS}$ ratio decreases in a factor of 10 . The $\mathrm{SiO}$ and HNCO line profiles for the $20 \mathrm{~km} \mathrm{~s}^{-1}$ GMC are very similar, peaking at the same velocities as one approaches the center. However, the high-velocity gas of the CND only appears in the $\mathrm{SiO}$ emission (see the middle column of Fig. 11).

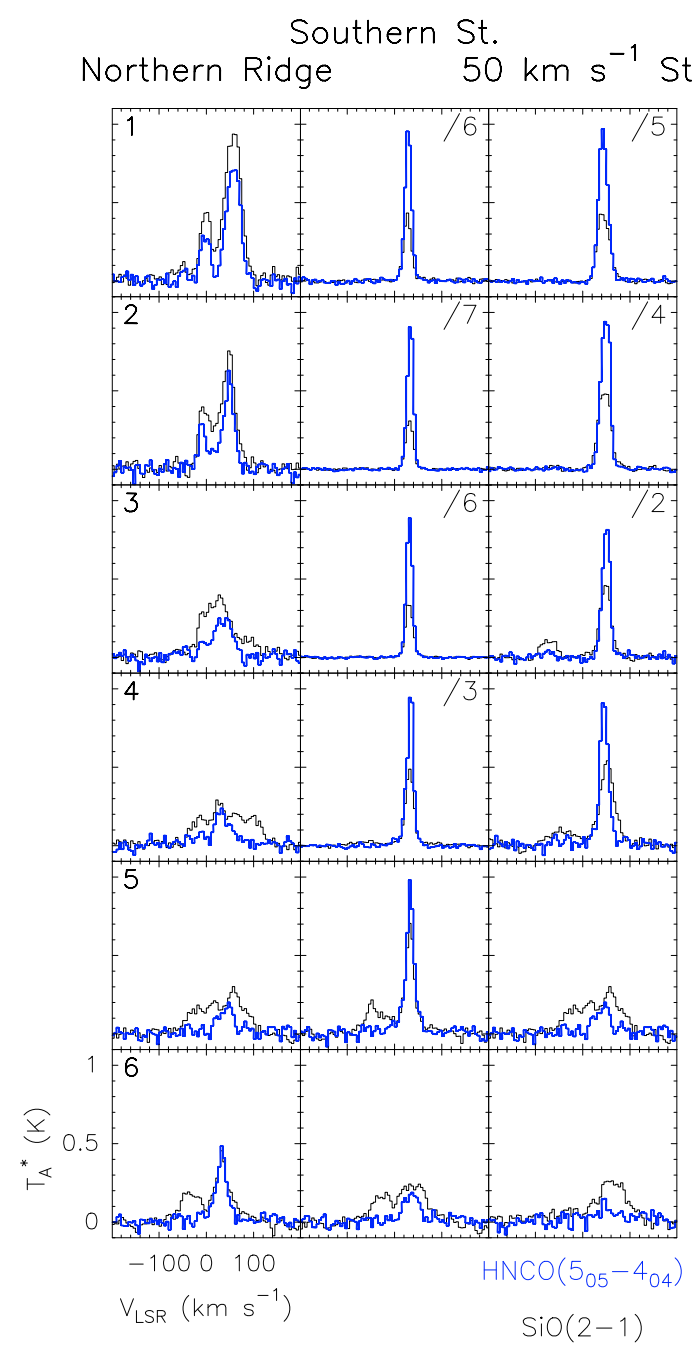

Fig. 11. $\mathrm{SiO}(2-1)$ (black line) and $\mathrm{HNCO}(5-4)$ (thick blue line) selected spectra taken along the Northern Ridge (left column), Southern Streamer (middle column) and $50 \mathrm{~km} \mathrm{~s}^{-1}$ Streamer (right column). In the maps at the top of Fig. 10, the areas where the spectra have been obtained can be seen as white open squares. Their numbers appear in the upper-left corner of each spectrum of the left column. The intensity of $\mathrm{SiO}(2-1)$ and $\mathrm{HNCO}(5-4)$ have been divided in some cases by a factor shown in the upper-right corner of the corresponding panel.

No velocity gradient has been detected along the Southern Streamer (Ho et al. 1991); therefore, if gas is accelerated along it, it must occur mainly perpendicular to the LOS, which complicates the connection between the CND and the $20 \mathrm{~km} \mathrm{~s}^{-1}$ GMC, since the GMC is located in front of the nucleus (Güsten \& Downes 1980; Park et al. 2004). Montero-Castaño et al. (2009) have compared the HCN(4-3)/HCN(1-0) intensity ratio map of the central region, which traces highly excited material in the CND, with the $\mathrm{NH}_{3}(3,3)$ and $(6,6)$ observations (Herrnstein \& Ho 2005), which trace the Southern Streamer and very warm gas, respectively. They find that at the location where the northeast part of the Southern Streamer and the southeastern part of the CND meet, the strongest peak in the $\mathrm{NH}_{3}(6,6)$ emission and a region of high $\mathrm{HCN}(4-3) / \mathrm{HCN}(1-0)$ ratio are found. Therefore, they propose that the impact of the Southern Streamer with the CND caused the infall of material toward the black hole, which is responsible for the highly excited gas found as we approach the center. Additionally, Sato \& Tsuboi (2008), from interferometric observations, find an increase in 
the $\mathrm{SiO}(2-1) / \mathrm{H}^{13} \mathrm{CO}^{+}(1-0)$ ratio at the possible interaction site between the southeastern region of the CND and the Southern Streamer, together with larger line widths. Another probe for shocks in this possible interaction site is the $36 \mathrm{GHz}$ methanol maser recently found by Sjouwerman et al. (2010) (see their Fig. 2) with a velocity of $22 \mathrm{~km} \mathrm{~s}^{-1}$ (these Class I masers are collisionally pumped and considered as typical signposts of shock-excited material). Moreover, Lee et al. (2008) claim that the SNR Sgr A East drives shocks into the northernmost part of the Southern Streamer and the southern part of the CND, pushing the gas toward us. These interactions with the SNR make the connection between the Southern Streamer and the CND possible. Therefore, the decrease in the $\mathrm{HNCO} / \mathrm{SiO}$ ratio as the Southern Streamer approaches the nuclear region confirms the interaction between the Southern Streamer and the CND.

- The $50 \mathrm{~km} \mathrm{~s}^{-1}$ Streamer: this ridge of gas extending westward from the $50 \mathrm{~km} \mathrm{~s}^{-1}$ GMC was first detected by Szczepanski et al. (1991) in methanol and was also observed in $\mathrm{HCN}(3-2)$ by Marshall et al. (1995). This streamer could be a possible connection between the $50 \mathrm{~km} \mathrm{~s}^{-1} \mathrm{GMC}$ and the northern lobe of the CND. Our data show a decrease in the $\mathrm{HNCO} / \mathrm{SiO}$ ratio that would indicate that this streamer is approaching the nucleus; however, if we compare the $\mathrm{SiO}(2-1)$ and $\mathrm{HNCO}(5-4)$ spectra extracted from the possible interaction region between the streamer and the CND (spectrum number 4 in the right column in Fig. 11), it seems that both molecules are tracing different gas components. The $\mathrm{HNCO}(5-4)$ line is tracing the $50 \mathrm{~km} \mathrm{~s}^{-1}$ GMC with very similar profiles to spectra with numbers $<4$, which sample the inner region of this molecular cloud. All of these $\mathrm{HNCO}$ profiles do not show line widths that are too broad, $\sim(19-23) \mathrm{km} \mathrm{s}^{-1}$. In contrast, the $\mathrm{SiO}(2-1)$ lines taken at the same positions show an increase in the line widths from $\sim(27-31) \mathrm{km} \mathrm{s}^{-1}$ (spectra with numbers $<4$ ) to $37 \mathrm{~km} \mathrm{~s}^{-1}$ (spectrum number 4 ). Moreover, the central velocity of the $\mathrm{SiO}(2-1)$ line is slightly redshifted with respect to the $\mathrm{HNCO}(5-4)$ line in spectrum number 4.

Marshall et al. (1995) do not detect any velocity gradient along the feature, and considering that the GMC is located behind the SNR Sgr A East, the connection is unlikely unless part of this GMC lies in the same plane as the northern lobe of the CND. In that case, the gas would run perpendicular to the LOS and no velocity gradient would be expected (Marshall et al. 1995). They suggest that this apparent connection could also be due to the overlapping of material from the GMC and the $\mathrm{CND}$, as their $\mathrm{HCN}(4-3)$ maps suggest. Therefore, in this case, the decrease in the $\mathrm{HNCO} / \mathrm{SiO}$ ratio could be produced by the blending of two gas components and not to a real interaction: the $50 \mathrm{~km} \mathrm{~s}^{-1} \mathrm{GMC}$, where the HNCO is more prominent than the $\mathrm{SiO}$ line, and gas from the $\mathrm{CND}$, where the HNCO line disappears because of photodissociation from the central UV radiation and $\mathrm{SiO}$ emission is broadened by shocks.

There are two other features, Cloud A and the Western Streamer, that do not seem to be connected with the CND, but their low $\mathrm{HNCO} / \mathrm{SiO}$ ratio indicates that they are close to the nuclear region. Both features seem to be related to the SNR Sgr A East:

- Cloud A: this cloud was previously observed by Genzel et al. (1990) in $\mathrm{C}^{18} \mathrm{O}(2-1)$, and mapped by Sutton et al. (1990) in CO(3-2) and Serabyn et al. (1992) in CS(5-4) and CS(7-6). In addition, Serabyn et al. (1992) found a redshifted emission $\left(80-85 \mathrm{~km} \mathrm{~s}^{-1}\right)$ just to the south of Cloud A. Both high-velocity emission components lie just inside of the compressed ridge of the $50 \mathrm{~km} \mathrm{~s}^{-1} \mathrm{GMC}$, which suggests that the molecular gas of this GMC had been accelerated to very high velocities by the expansion of Sgr A East. The accelerated molecular gas appears on the front and the back sides of the expanding radio shell. Wright et al. (2001) support this scenario from their interferometric $\mathrm{HCO}^{+}$and $\mathrm{HCN}$ maps, together with other near blue and redshifted clumps of gas inside of the compressed ridge. A different interpretation was proposed by Liszt \& Burton (1995), who detected this feature with very strong emission in $\mathrm{CO}(3-2)$ at $v \leq-40 \mathrm{~km} \mathrm{~s}^{-1}$, but they considered this feature as an extension of the high-longitude forbidden-velocity part of the CND, as they see a connection between it and the emission arising from the southwestern region of the CND, concluding that Cloud A was not an isolated feature.

From the declination-velocity cut made through it (panel $\mathrm{RA}=\left[45^{\prime \prime}, 105^{\prime \prime}\right]$ in Fig. 8), this cloud does not appear to be kinematically connected to either the $50 \mathrm{~km} \mathrm{~s}^{-1} \mathrm{GMC}$ or to any other gas component. The $\mathrm{SiO} / \mathrm{CS}$ and $\mathrm{HNCO} / \mathrm{SiO}$ ratios in this cloud ( $\sim 0.7$ and $\leq 0.22$, respectively; see Fig. 10$)$ suggest there are shocks, as well as UV-radiation, which also explain the lack of $\mathrm{NH}_{3}$ and $1.2 \mathrm{~mm}$ dust emission, since the ammonia molecules and dust grains are destroyed by photodissociation and shocks, respectively (see Fig. 9 of McGary et al. 2001). Shocks could be caused by the expansion of Sgr A East SNR into this cloud, whereas UV-radiation could originat in the Central cluster, locating this cloud close to the nuclear region. The SiO/CS ratio in Cloud $\mathrm{A}$ is one of the highest found in the region, only exceeded by the southwest lobe of the CND. This fact could be explained by the geometry of the shock, that could be almost along the LOS. Additional support for the SNR interaction scenario comes from the central velocities of the $\mathrm{SiO}(2-1)$ and $\mathrm{CS}(1-0)$ profiles. The $\mathrm{SiO}(2-1)$ central velocity is offset by $-11 \pm 3 \mathrm{~km} \mathrm{~s}^{-1}$ with respect to that of CS(1-0), indicating that Cloud A is pushed toward us by the SNR Sgr A East, if we consider that $\mathrm{SiO}$ traces more efficiently the shocked gas, whereas $\mathrm{CS}$ traces more quiescent gas. Moreover, Cloud A is detected in the 18-cm OH absorption maps of Karlsson et al. (2003) (in the 1665 and $1667 \mathrm{MHz}$ lines), which locates it in front of the continuum emission of the SNR, consistent with our finding.

- The Western Streamer: this feature is a long curved filamentary structure that closely matches the western edge of Sgr A East. McGary et al. (2001) reports a velocity gradient of $1 \mathrm{~km} \mathrm{~s}^{-1} \operatorname{arcsec}^{-1}$ along its $150^{\prime \prime}$ length. The southern half has a negative velocity whereas the northern half has a positive velocity, consistent with a ridge of gas highly inclined to the LOS that is pushed outward by the expansion of the SNR. The northern part of the streamer would be located on the far side of the SNR producing the redshifted emission, whereas the southern part would be located on the front side of the shell producing the blueshifted velocities (McGary et al. 2001; Lee et al. 2008). The $\mathrm{SiO} / \mathrm{CS}$ ratios in this streamer present intermediate values of $0.3-0.4$. On the other hand, the $\mathrm{HNCO} / \mathrm{SiO}$ ratios seem to be low $(0.3-0.4)$ with very low-intensity emission of HNCO, just slightly over the rms noise. This enhancement in the SiO/CS ratios could be attributed to shocks caused by the expansion of the SNR Sgr A East into the molecular filament. The smaller enhancement of the ratio with respect to the enhancement observed in the southwestern lobe of the CND and Cloud A could be due to the geometry of the interaction. In this case it must be highly inclined and orthogonal to the LOS. The Western Streamer is not detected in the $1.2 \mathrm{~mm}$ dust emission map (see Fig. 9 of McGary et al. 2001). These authors propose that the gas of the streamer could originate closer to the nucleus, where most of the dust is destroyed by UV-photons from the central cluster, and then pushed outwards by the SNR. Our data suggest that the dust destruction is very likely caused by shocks. Molecular gas of the 
Western Streamer also presents the highest kinetic temperatures in the central $10 \mathrm{pc}$, even showing $\mathrm{NH}_{3}(6,6)$ emission that traces gas at $\geq 100 \mathrm{~K}$ (Herrnstein \& Ho 2005).

In summary, the $\mathrm{HNCO} / \mathrm{SiO}$ intensity ratio seems to be an excellent tool for locating the different gas components relative to the nucleus. While the $50 \mathrm{~km} \mathrm{~s}^{-1}$ Streamer seems to be due to the overlapping of two different gas components $\left(\mathrm{CND}+50 \mathrm{~km} \mathrm{~s}^{-1} \mathrm{GMC}\right.$ gas $)$, the Southern Streamer seems to be feeding the CND from the $20 \mathrm{~km} \mathrm{~s}^{-1} \mathrm{GMC}$, whereas the Northern Ridge remains a good candidate to also feed the CND, possible connecting the $-30 \mathrm{~km} \mathrm{~s}^{-1}$ molecular cloud with the central region. The Western Streamer and Cloud A seem to be associated with the expansion of the SNR Sgr A East and located close to the nuclear region. This streamer would arise from the western border of the SNR interacting with the molecular gas, whereas Cloud A could be part of the $50 \mathrm{~km} \mathrm{~s}^{-1}$ GMC that has been pushed by the SNR toward us almost along the LOS.

\section{Conclusions}

We have presented maps of the central $12 \mathrm{pc}$ of the GC in the $J=2 \rightarrow 1$ transition of $\mathrm{SiO}$, the $J=5_{0,5} \rightarrow 4_{0,4}$ transition of $\mathrm{HNCO}$, and the $J=1 \rightarrow 0$ transition of $\mathrm{H}^{13} \mathrm{CO}^{+}, \mathrm{HN}^{13} \mathrm{C}$, and $\mathrm{C}^{18} \mathrm{O}$, observed with the IRAM-30 m telescope at Pico Veleta, with an angular resolution of $30^{\prime \prime}$.

The main results obtained from this work can be summarized as follow:

- The $\mathrm{SiO}(2-1)$ emission, observed in a velocity range of $[-125,130] \mathrm{km} \mathrm{s}^{-1}$, perfectly traces the CND, as well as all the features previously identified in other high-density molecular tracers. The $\mathrm{H}^{13} \mathrm{CO}^{+}(1-0)$ emission covers the same velocity range and follows the $\mathrm{SiO}$ morphology, except for the absorption features toward the radio continuum source Sgr A*.

- In contrast, the $\mathrm{HNCO}(5-4)$ molecular line shows emission in the narrowest velocity range $\left([-30,85] \mathrm{km} \mathrm{s}^{-1}\right)$. This molecular line presents the strongest emission of all the observed lines; however, its emission is concentrated in the GMCs and the Molecular Ridge, quickly disappearing as one approaches the Central cluster. Similar behavior is shown by the $\mathrm{HN}^{13} \mathrm{C}(1-0)$ emission.

- The $\mathrm{C}^{18} \mathrm{O}(1-0)$ emission is affected by contamination with foreground gas due to its lower critical density.

- Emission mostly coming from $\mathrm{NH}_{2} \mathrm{CHO}\left(5_{1,4}-4_{1,3}\right)$ has been detected toward the GMCs and the Molecular Ridge.

- Using the LVG approximation, volume densities of $\sim 10^{5} \mathrm{~cm}^{-3}$ assuming $T_{\mathrm{K}}=50 \mathrm{~K}$ are derived for all the gas components, expect for the the $20 \mathrm{~km} \mathrm{~s}^{-1} \mathrm{GMC}$, which could be either less dense or colder. In general, the highest densities are found toward the CND and Western Streamer, and the lowest ones toward the two GMCs.

- Hydrogen column densities have been derived from the highdensity tracer CS, obtaining values of $0.5-6 \times 10^{22} \mathrm{~cm}^{-2}$, with the highest value found toward the $50 \mathrm{~km} \mathrm{~s}^{-1}$ GMC.

- The HNCO molecule shows the strongest contrast in column density and fractional abundance, with factors of $\geq 60$ $\left(\leq 0.8-50 \times 10^{13} \mathrm{~cm}^{-2}\right)$ and $28\left(\sim 0.7-19 \times 10^{-9}\right)$, respectively. The highest values are found toward the cores of the GMCs, whereas the lowest ones are derived toward the positions associated with the CND. A similar behavior is also found for $\mathrm{HN}^{13} \mathrm{C}$, with abundances $\sim 0.06-1.3 \times 10^{-9}$.

- SiO abundances do not follow the HNCO trend $\left(0.20-2.4 \times 10^{-9}\right)$, with high values at the cores of the
GMCs, as well as toward the $\mathrm{CND}$, whereas $\mathrm{H}^{13} \mathrm{CO}^{+}$ shows the smallest variation in its fractional abundance $\left(0.05-0.4 \times 10^{-9}\right)$. Thus, the observed species can be grouped into those whose abundances decrease (HNCO and $\mathrm{HN}^{13} \mathrm{C}$ ), and those whose abundances remain similar or are enhanced toward the nuclear region $\left(\mathrm{SiO}\right.$ and $\left.\mathrm{H}^{13} \mathrm{CO}^{+}\right)$.

- From the comparison of the abundances derived toward the 12 central parsecs of our Galaxy with those of prototypical Galactic sources, one can conclude that non-dissociative C-shocks with velocities between $20-30 \mathrm{~km} \mathrm{~s}^{-1}$, able to eject $\mathrm{Si} / \mathrm{SiO}$ from the grain mantles to the gas phase, are responsible for maintaining the high $\mathrm{SiO}$ abundances found in the CND and the GMCs. HNCO could also be locked in grain mantles and directly realeased to the gas phase by the $\mathrm{C}$-shocks. However, once in the gas phase, HNCO molecules are not well-shielded from the UV radiation coming from the Central cluster and would be rapidly destroyed, leading to the low abundances found in the CND. This strong UV field also seems to be responsible for the low abundances of $\mathrm{HN}^{13} \mathrm{C}$ measured toward the $\mathrm{CND}$, as high temperatures could favor neutral-neutral reactions leading to the destruction of HNC molecules. The small gradient found in the $\mathrm{H}^{13} \mathrm{CO}^{+}$abundance from the CND to the $50 \mathrm{~km} \mathrm{~s}^{-1} \mathrm{GMC}$ could be explained by a decrease of one order of magnitude in the ionization rate with respect to the "standard" value toward the $50 \mathrm{~km} \mathrm{~s}^{-1} \mathrm{GMC}$.

- Ratios between $\mathrm{SiO}$ (which traces shocked gas), HNCO (shocked and/or photodissociated gas), and CS (quiescent dense gas) emissions provide an excellent tool for studing the possible connections and the $3 \mathrm{D}$ arrangement of the different features found in the central region of the Galaxy. From their comparison, we conclude that not only is the CND affected by UV radiation, but also the Western Streamer and Cloud A. The Southern Streamer and the Northern Ridge could be feeding the CND; however, the $50 \mathrm{~km} \mathrm{~s}^{-1}$ Streamer, composed of two overlapping components, does not seem to be connected with the CND.

Acknowledgements. The authors would like to thank Prof. M. Tsuboi and D. M. Montero-Castaño for kindly providing their CS(1-0) and $\operatorname{HCN}(4-3)$ data, respectively. We also thank the referee for the suggestions. This work was supported by the Spanish Ministerio de Ciencia e Innovación under project ESP2007-65812-C02-01.

\section{References}

Amo-Baladrón, M. A., Martín-Pintado, J., Morris, M. R., Muno, M. P., \& Rodríguez-Fernández, N. J. 2009, ApJ, 694, 943

Bayet, E., Aladro, R., Martín, S., Viti, S., \& Martín-Pintado, J. 2009, ApJ, 707, 126

Becklin, E. E., Gatley, I., \& Werner, M. W. 1982, ApJ, 258, 135

Bisschop, S. E., Jørgensen, J. K., van Dishoeck, E. F., \& de Wachter, E. B. M. 2007, A\&A, 465, 913

Bradford, C. M., Stacey, G. J., Nikola, T., et al. 2005, ApJ, 623, 866

Burton, M. G., Hollenbach, D. J., \& Tielens, A. G. G. M. 1990, ApJ, 365, 620

Christopher, M. H., Scoville, N. Z., Stolovy, S. R., \& Yun, M. S. 2005, ApJ, 622, 346

Coil, A. L., \& Ho, P. T. P. 1999, ApJ, 513, 752

Coil, A. L., \& Ho, P. T. P. 2000, ApJ, 533, 245

Cummins, S. E., Linke, R. A., \& Thaddeus, P. 1986, ApJS, 60, 819

Dahmen, G., Hüttemeister, S., Wilson, T. L., et al. 1997, A\&AS, 126, 197

Davidson, J. A., Werner, M. W., Wu, X., et al. 1992, ApJ, 387, 189

Dent, W. R. F., Matthews, H. E., Wade, R., \& Duncan, W. D. 1993, ApJ, 410, 650

Downes, D., Radford, S. J. E., Guilloteau, S., et al. 1992, A\&A, 262, 424

Farquhar, P. R. A., Millar, T. J., \& Herbst, E. 1994, MNRAS, 269, 641

Figer, D. F. 2008, Proc. IAU Symp., 250, 247

Garay, G., Köhnenkamp, I., Bourke, T. L., Rodríguez, L. F., \& Lehtinen, K. K. 1998, ApJ, 509, 768 
Garden, R. P., \& Carlstrom, J. E. 1992, ApJ, 392, 602

Garrod, R. T., Weaver, S. L. W., \& Herbst, E. 2008, ApJ, 682, 283

Gatley, I., Jones, T. J., Hyland, A. R., et al. 1986, MNRAS, 222, 299

Geballe, T. R., Baas, F., \& Wade, R. 1989, A\&A, 208, 255

Genzel, R., Crawford, M. K., Townes, C. H., \& Watson, D. M. 1985, ApJ, 297,

Genzel, R., Stacey, G. J., Harris, A. I., et al. 1990, ApJ, 356, 160

Ghez, A. M., Salim, S., Hornstein, S. D., et al. 2005, ApJ, 620, 744

Girart, J. M., Ho, P. T. P., Rudolph, A. L., et al. 1999, ApJ, 522, 921

Goicoechea, J. R., Pety, J., Gerin, M., et al. 2006, A\&A, 456, 565

Goicoechea, J. R., Pety, J., Gerin, M., Hily-Blant, P., \& Le Bourlot, J. 2009, A\&A, 498, 771

Güsten, R., \& Downes, D. 1980, A\&A, 87, 6

Güsten, R., \& Henkel, C. 1983, A\&A, 125, 136

Güsten, R., Walmsley, C. M., \& Pauls, T. 1981, A\&A, 103, 197

Güsten, R., Genzel, R., Wright, M. C. H., et al. 1987, ApJ, 318, 124

Harris, A. I., Jaffe, D. T., Silber, M., \& Genzel, R. 1985, ApJ, 294, L93

Hasegawa, T. I., \& Herbst, E. 1993, MNRAS, 263, 589

Henkel, C., Chin, Y., Mauersberger, R., \& Whiteoak, J. B. 1998, A\&A, 329, 443

Herrnstein, R. M., \& Ho, P. T. P. 2002, ApJ, 579, L83

Herrnstein, R. M., \& Ho, P. T. P. 2005, ApJ, 620, 287

Hirota, T., Yamamoto, S., Mikami, H., \& Ohishi, M. 1998, ApJ, 503, 717

Ho, P. T. P., Ho, L. C., Szczepanski, J. C., Jackson, J. M., \& Armstrong, J. T. 1991, Nature, 350, 309

Hogerheijde, M. R., Jansen, D. J., \& van Dishoeck, E. F. 1995, A\&A, 294, 792

Hogerheijde, M. R., van Dishoeck, E. F., Blake, G. A., \& van Langevelde, H. J. 1998, ApJ, 502, 315

Hudson, R. L., Moore, M. H., \& Gerakines, P. A. 2001, ApJ, 550, 1140

Hüttemeister, S., Wilson, T. L., Bania, T. M., \& Martin-Pintado, J. 1993, A\&A, 280,255

Hüttemeister, S., Dahmen, G., Mauersberger, R., et al. 1998, A\&A, 334, 646

Iglesias, E. 1977, ApJ, 218, 697

Irvine, W. M., Goldsmith, P. F., \& Hjalmarson, A. 1987, in Interstellar Processes, ed. D. J. Hollenbach, \& H. A. Thronson Jr., Astrophys. Space Sci. Libr., 134, 561

Jackson, J. M., Armstrong, J. T., \& Barrett, A. H. 1984, ApJ, 280, 608

Jackson, J. M., Geis, N., Genzel, R., et al. 1993, ApJ, 402, 173

Jansen, D. J., Spaans, M., Hogerheijde, M. R., \& van Dishoeck, E. F. 1995 A\&A, 303, 541

Jiménez-Serra, I., Martín-Pintado, J., Rodríguez-Franco, A., \& Martín, S. 2005, ApJ, 627, L121

Jiménez-Serra, I., Caselli, P., Martín-Pintado, J., \& Hartquist, T. W. 2008, A\&A, 482,549

Karlsson, R., Sjouwerman, L. O., Sandqvist, A., \& Whiteoak, J. B. 2003, A\&A, 403, 1011

Krabbe, A., Genzel, R., Eckart, A., et al. 1995, ApJ, 447

Kuan, Y., \& Snyder, L. E. 1996, ApJ, 470, 981

Lee, S., Pak, S., Choi, M., et al. 2008, ApJ, 674, 247

Lindqvist, M., Sandqvist, A., Winnberg, A., Johansson, L. E. B., \& Nyman, L.-A. 1995, A\&AS, 113, 257

Liszt, H. S., \& Burton, W. B. 1995, ApJS, 98, 679

Liszt, H. S., Burton, W. B., \& van der Hulst, J. M. 1985, A\&A, 142, 237

Lo, K. Y., \& Claussen, M. J. 1983, Nature, 306, 647

Lugten, J. B., Genzel, R., Crawford, M. K., \& Townes, C. H. 1986, ApJ, 306, 691

Maeda, Y., Baganoff, F. K., Feigelson, E. D., et al. 2002, ApJ, 570, 671

Marcelino, N. 2007, Ph.D. Thesis, Universidad de Granada

Marcelino, N., Cernicharo, J., Tercero, B., \& Roueff, E. 2009, ApJ, 690, L27

Marr, J. M., Wright, M. C. H., \& Backer, D. C. 1993, ApJ, 411, 667

Marshall, J., Lasenby, A. N., \& Harris, A. I. 1995, MNRAS, 277, 594

Martín, S., Mauersberger, R., Martín-Pintado, J., Henkel, C., \& García-Burillo, S. 2006, ApJS, 164, 450

Martín, S., Requena-Torres, M. A., Martín-Pintado, J., \& Mauersberger, R. 2008, ApJ, 678, 245

Martín, S., Martín-Pintado, J., \& Mauersberger, R. 2009a, ApJ, 694, 610

Martín, S., Martín-Pintado, J., \& Viti, S. 2009b, ApJ, 706, 1323

Martín-Pintado, J., Bachiller, R., \& Fuente, A. 1992, A\&A, 254, 315

Martín-Pintado, J., de Vicente, P., Fuente, A., \& Planesas, P. 1997, ApJ, 482

Martín-Pintado, J., de Vicente, P., Rodríguez-Fernández, N. J., Fuente, A., \& Planesas, P. 2000, A\&A, 356, L5

McGary, R. S., Coil, A. L., \& Ho, P. T. P. 2001, ApJ, 559, 326
Meier, D. S., \& Turner, J. L. 2005, ApJ, 618, 259

Mezger, P. G., Zylka, R., Salter, C. J., et al. 1989, A\&A, 209, 337

Minh, Y. C., \& Irvine, W. M. 2006, New Astron., 11, 594

Montero-Castaño, M., Herrnstein, R. M., \& Ho, P. T. P. 2009, ApJ, 695, 1477

Nummelin, A., Bergman, P., Hjalmarson, Å., et al. 2000, ApJS, 128, 213

Oka, T., Geballe, T. R., Goto, M., Usuda, T., \& McCall, B. J. 2005, ApJ, 632 882

Okumura, S. K., Ishiguro, M., Fomalont, E. B., et al. 1991, ApJ, 378, 127

Park, S., Muno, M. P., Baganoff, F. K., et al. 2004, ApJ, 603, 548

Pedlar, A., Anantharamaiah, K. R., Ekers, R. D., et al. 1989, ApJ, 342, 769

Persson, C. M., Olofsson, A. O. H., Koning, N., et al. 2007, A\&A, 476, 807

Pineau des Forets, G., Roueff, E., \& Flower, D. R. 1990, MNRAS, 244, 668

Rawlings, J. M. C., Taylor, S. D., \& Williams, D. A. 2000, MNRAS, 313, 461

Reid, M. J. 1993, ARA\&A, 31, 345

Requena-Torres, M. A., Martín-Pintado, J., Rodríguez-Franco, A., et al. 2006, A\&A, 455, 971

Roberts, D. A., \& Goss, W. M. 1993, ApJS, 86, 133

Rodríguez-Fernández, N. J., Martín-Pintado, J., Fuente, A., et al. 2001, A\&A, 365,174

Rodriguez-Fernandez, N., Tafalla, M., Gueth, F., \& Bachiller, R. 2010, A\&A, 516, A98

Sanders, R. H. 1998, MNRAS, 294, 35

Sandqvist, A., Larsson, B., Hjalmarson, A., et al. 2008, A\&A, 482, 849

Sato, M. T., \& Tsuboi, M. 2008, J. Phys. Conf. Ser., 131, 012033

Sato, F., Hasegawa, T., Dobashi, K., Kandori, R., \& Whiteoak, J. B. 2002, in 8th Asian-Pacific Regional Meeting, Vol. II, ed. S. Ikeuchi, J. Hearnshaw, \& T. Hanawa, 205

Schilke, P., Walmsley, C. M., Pineau Des Forets, G., et al. 1992, A\&A, 256, 595

Schilke, P., Pineau des Forêts, G., Walmsley, C. M., \& Martín-Pintado, J. 2001, A\&A, 372, 291

Schinnerer, E., Böker, T., Meier, D. S., \& Calzetti, D. 2008, ApJ, 684, L21

Serabyn, E., \& Lacy, J. H. 1985, ApJ, 293, 445

Serabyn, E., \& Güsten, R. 1987, A\&A, 184, 133

Serabyn, E., Güsten, R., Walmsley, J. E., Wink, J. E., \& Zylka, R. 1986, A\&A, 169,85

Serabyn, E., Lacy, J. H., \& Achtermann, J. M. 1992, ApJ, 395, 166

Serabyn, E., Keene, J., Lis, D. C., \& Phillips, T. G. 1994, ApJ, 424, L95

Shukla, H., Yun, M. S., \& Scoville, N. Z. 2004, ApJ, 616, 231

Sjouwerman, L. O., \& Pihlström, Y. M. 2008, ApJ, 681, 1287

Sjouwerman, L. O., Pihlström, Y. M., \& Fish, V. L. 2010, ApJ, 710, L111

Sternberg, A., \& Dalgarno, A. 1995, ApJS, 99, 565

Sutton, E. C., Danchi, W. C., Jaminet, P. A., \& Masson, C. R. 1990, ApJ, 348, 503

Szczepanski, J. C., Ho, P. T. P., \& Gusten, R. 1991, in Atoms, Ions and Molecules: New Results in Spectral Line Astrophysics, ed. A. D. Haschick, \& P. T. P. Ho, ASP Conf. Ser., 16

Talbi, D., Ellinger, Y., \& Herbst, E. 1996, A\&A, 314, 688

Telesco, C. M., Davidson, J. A., \& Werner, M. W. 1996, ApJ, 456, 541

Tercero, B., Cernicharo, J., Pardo, J. R., \& Goicoechea, J. R. 2010, A\&A, 517, A96

Tideswell, D. M., Fuller, G. A., Millar, T. J., \& Markwick, A. J. 2010, A\&A, 510, A85

Tsuboi, M., Handa, T., \& Ukita, N. 1999, ApJS, 120, 1

Turner, B. E., Terzieva, R., \& Herbst, E. 1999, ApJ, 518, 699

Usero, A., García-Burillo, S., Martín-Pintado, J., Fuente, A., \& Neri, R. 2006, A\&A, 448, 457

van der Tak, F. F. S., Belloche, A., Schilke, P., et al. 2006, A\&A, 454, L99

van der Tak, F. F. S., Black, J. H., Schöier, F. L., Jansen, D. J., \& van Dishoeck, E. F. 2007, A\&A, 468, 627

van Dishoeck, E. F., Jansen, D. J., \& Phillips, T. G. 1993, A\&A, 279, 541

Viti, S., Natarajan, S., \& Williams, D. A. 2002, MNRAS, 336, 797

Vollmer, B., \& Duschl, W. J. 2002, A\&A, 388, 128

Wang, M., Henkel, C., Chin, Y., et al. 2004, A\&A, 422, 883

Wilson, T. L. 1999, Rep. Prog. Phys., 62, 143

Wright, M. C. H., Coil, A. L., McGary, R. S., Ho, P. T. P., \& Harris, A. I. 2001 , ApJ, 551, 254

Yusef-Zadeh, F., \& Morris, M. 1987, ApJ, 320, 545

Yusef-Zadeh, F., Stolovy, S. R., Burton, M., Wardle, M., \& Ashley, M. C. B. 2001, ApJ, 560, 749

Zinchenko, I., Henkel, C., \& Mao, R. Q. 2000, A\&A, 361, 1079

Zylka, R., Mezger, P. G., \& Wink, J. E. 1990, A\&A, 234, 133

Pages 24 to 29 are available in the electronic edition of the journal at http: //www . aanda.org 


\section{Appendix A: Velocity channel maps}

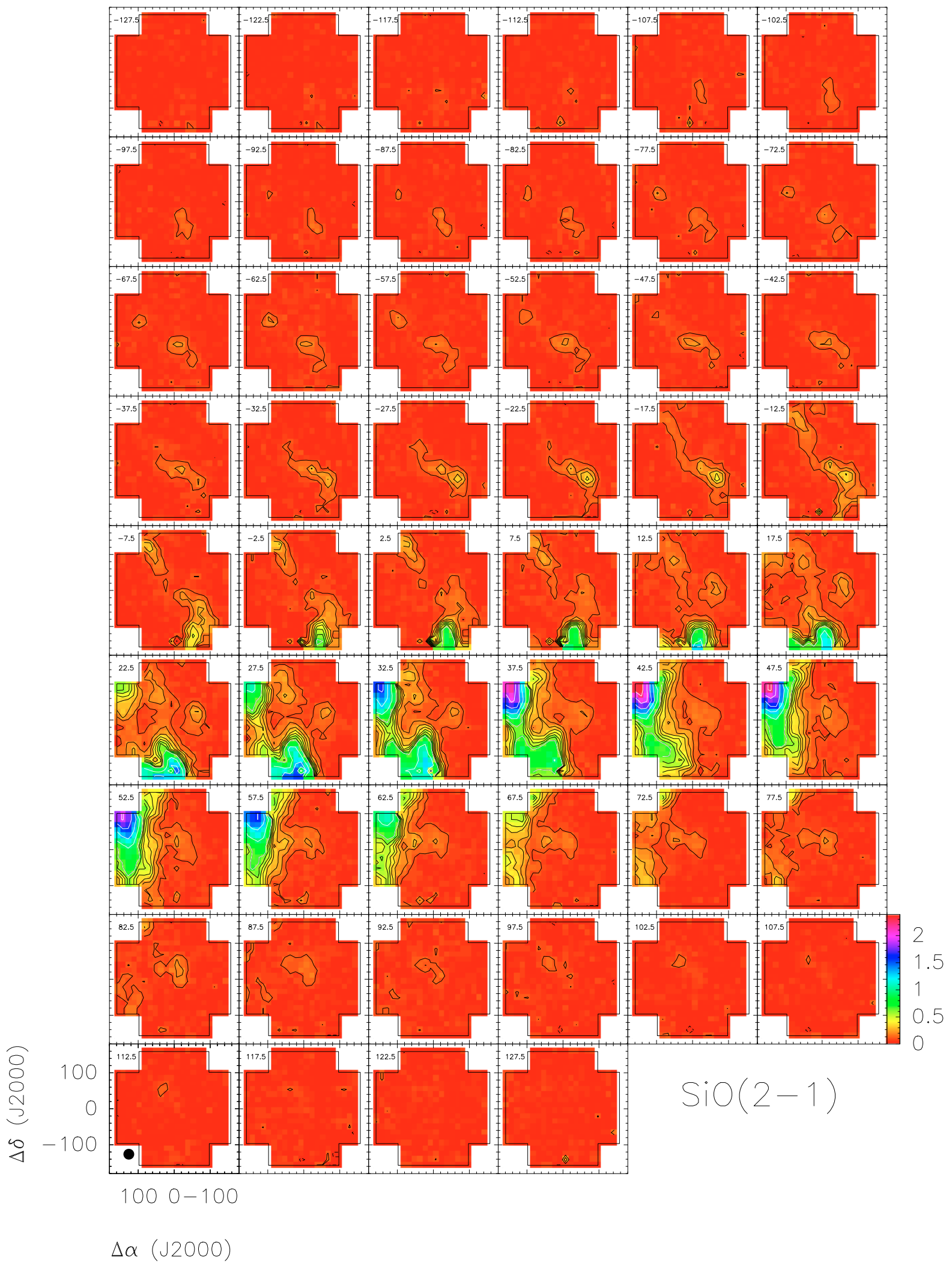

Fig. A.1. Velocity-channel maps of the $\mathrm{SiO}(2-1)$ emission. Black contour levels for the molecular line emission (in the $T_{\mathrm{A}}^{*}$ scale) are from $0.08 \mathrm{~K}$ $(3 \sigma)$ to $0.64 \mathrm{~K}$ in steps of $0.08 \mathrm{~K}$. White contour levels are from $0.64 \mathrm{~K}$ in steps of $0.32 \mathrm{~K}$. Dashed countours correspond to the $-3 \sigma$ level. Velocity channels range from -130 to $130 \mathrm{~km} \mathrm{~s}^{-1}$ by steps of $5 \mathrm{~km} \mathrm{~s}^{-1}$ (the velocity width per channel). The central velocities of the velocity-channel maps are shown in the upper-left corner of each panel. The wedge at the side shows the intensity scale of the $\mathrm{SiO}(2-1)$ emission. The beam size (30") is shown at the bottom-left corner. Sgr A* is the origin for the offset coordinates (in arcseconds). 
M. A. Amo-Baladrón et al.: Mapping the surrounding of Sgr A*

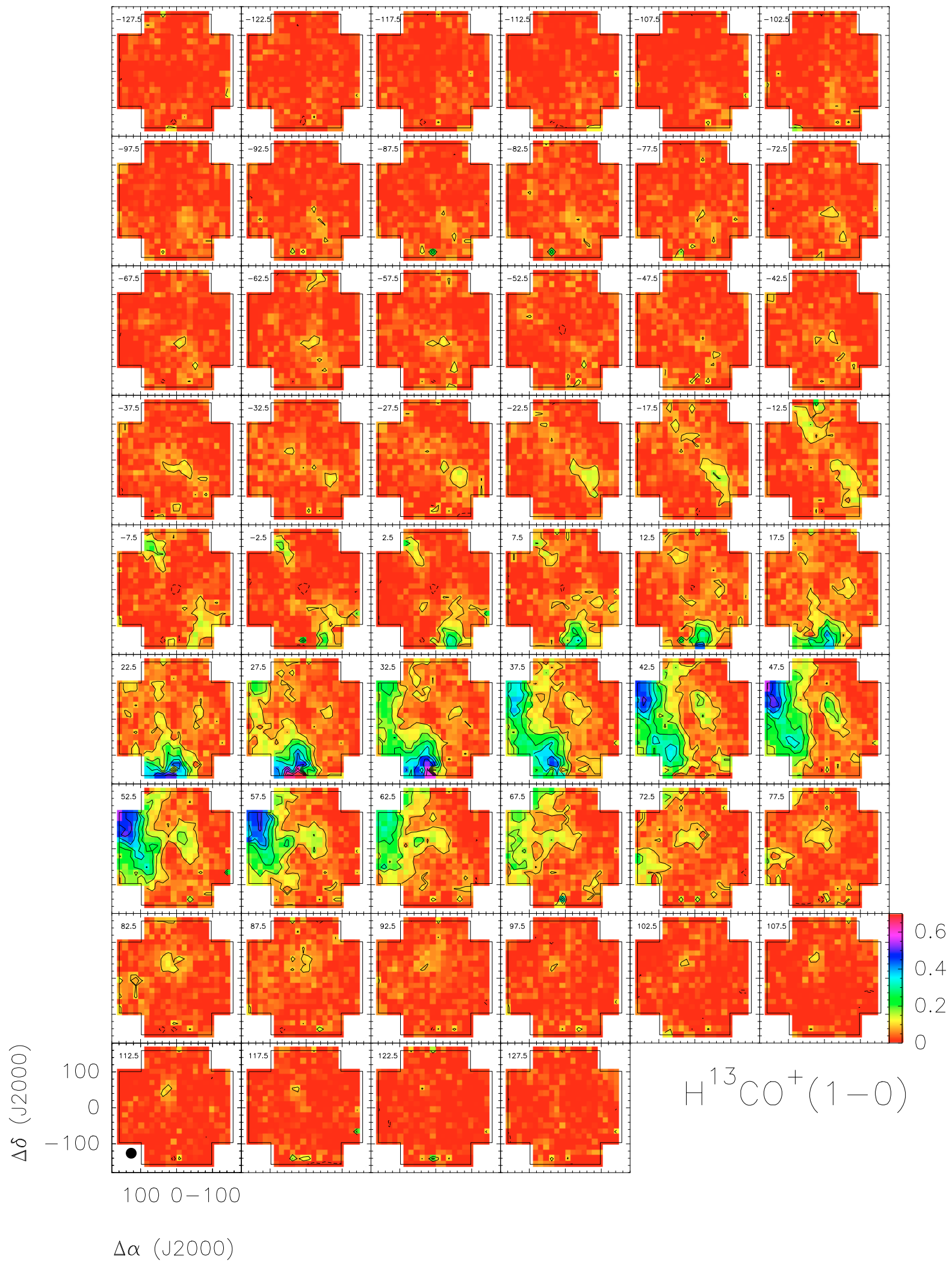

Fig. A.2. Velocity-channel maps of the $\mathrm{H}^{13} \mathrm{CO}^{+}(1-0)$ emission. Contour levels for the molecular line emission are from $0.08 \mathrm{~K}(3 \sigma)$ in steps of $0.08 \mathrm{~K}$. The other characteristics of the figure are the same as in Fig. A.1. 
A\&A 526, A54 (2011)

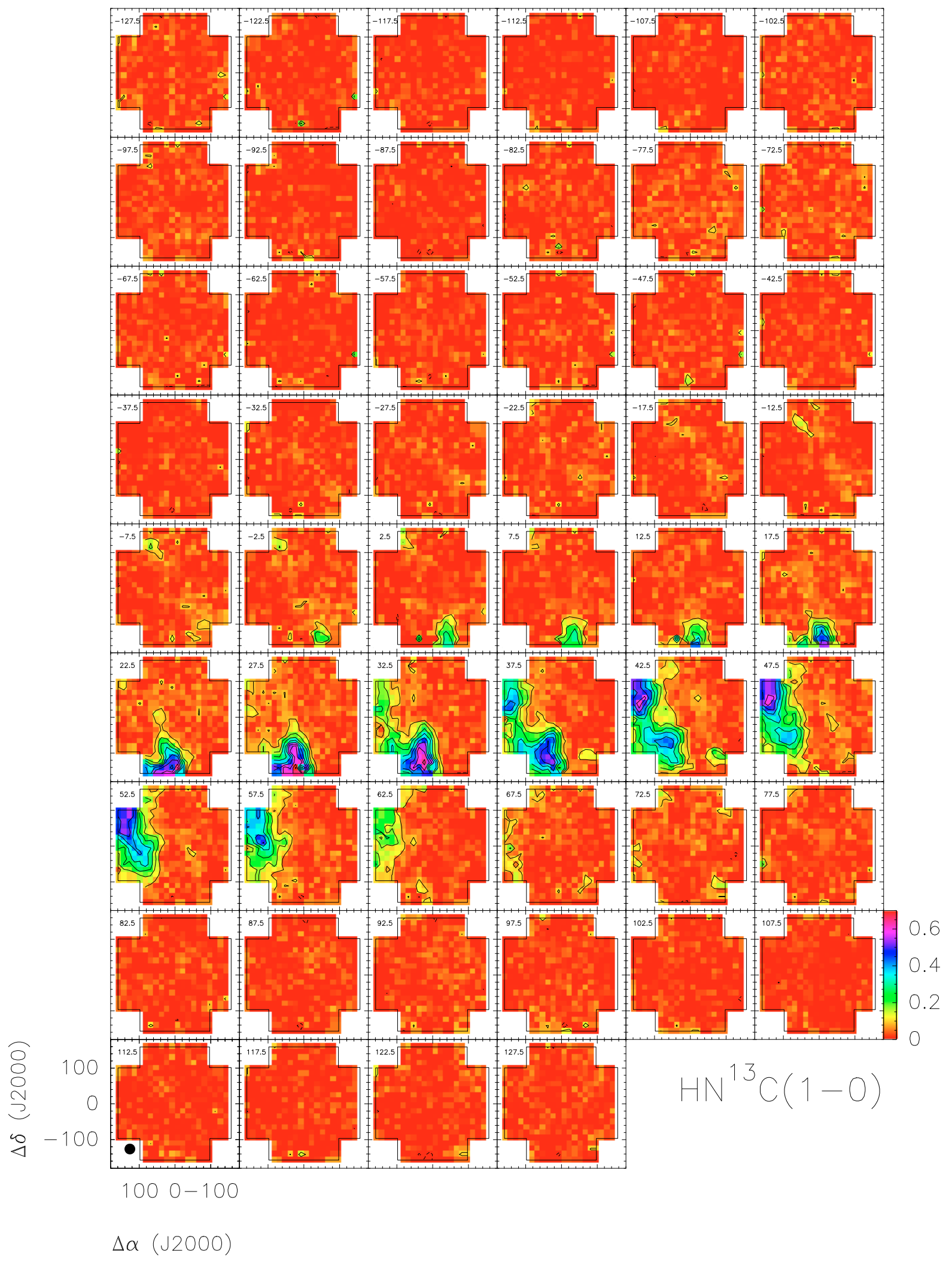

Fig. A.3. Same as Fig. A.2 but for the $\mathrm{HN}^{13} \mathrm{C}(1-0)$ emission. 
M. A. Amo-Baladrón et al.: Mapping the surrounding of Sgr A*

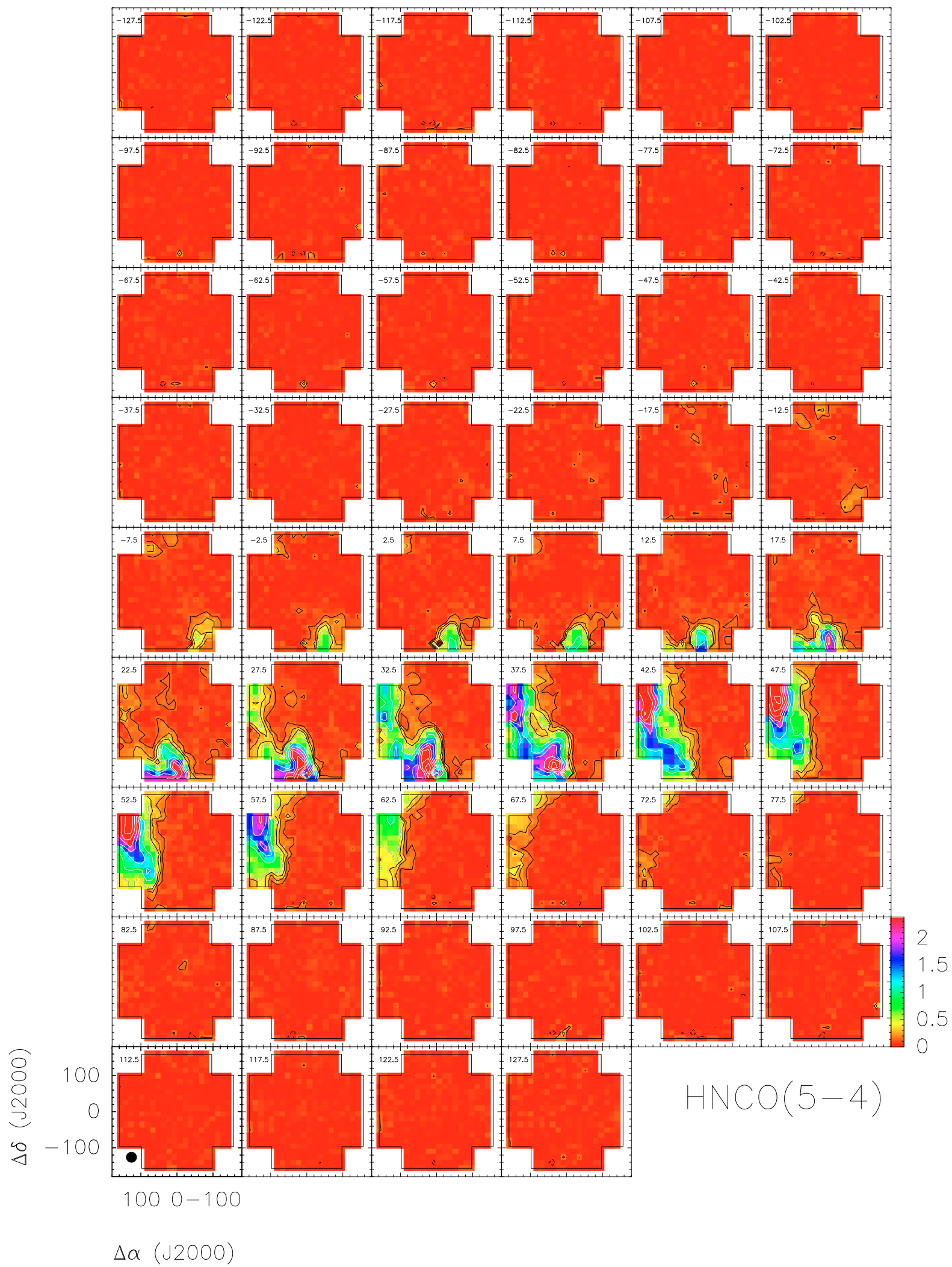

Fig. A.4. Velocity-channel maps of the $\operatorname{HNCO}\left(5_{0,5}-4_{0,4}\right)$ emission. Black contour levels for the molecular line emission are from $0.11 \mathrm{~K}(3 \sigma)$ to $0.44 \mathrm{~K}$ in steps of $0.11 \mathrm{~K}$. White contour levels are from $0.44 \mathrm{~K}$ in steps of $0.44 \mathrm{~K}$. In order to show more clearly the spatial distribution of the weak emission, the intensity scale has been saturated. The other characteristics of the figure are the same as in Fig. A.1. 
A\&A 526, A54 (2011)

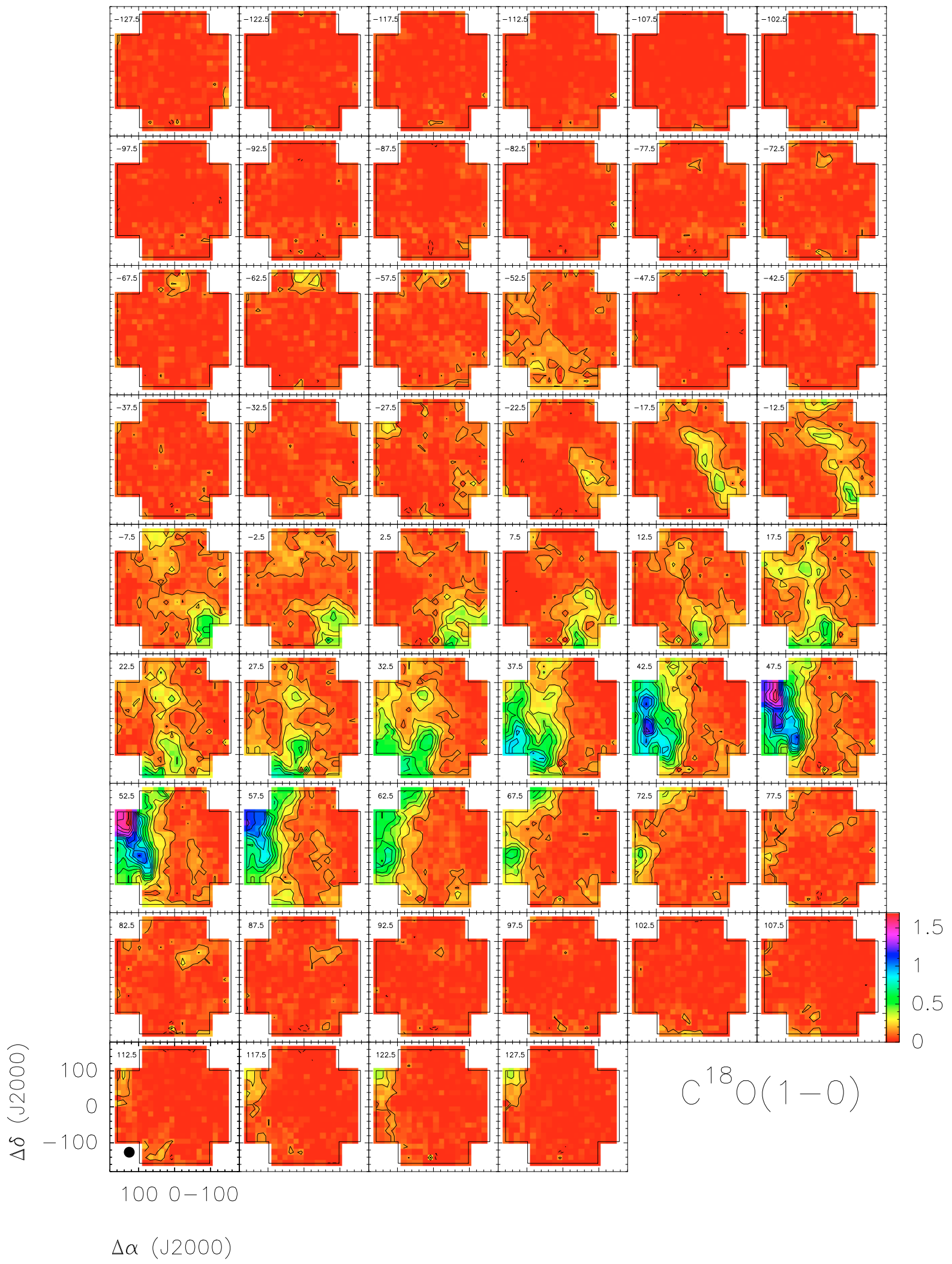

Fig. A.5. Velocity-channel maps of the $\mathrm{C}^{18} \mathrm{O}(1-0)$ emission. Contour levels for the molecular line emission are from $0.11 \mathrm{~K}(3 \sigma)$ in steps of $0.11 \mathrm{~K}$. The other characteristics of the figure are the same as in Fig. A.1. 
M. A. Amo-Baladrón et al.: Mapping the surrounding of Sgr A*

\section{Appendix B: Integrated intensity ratio maps}

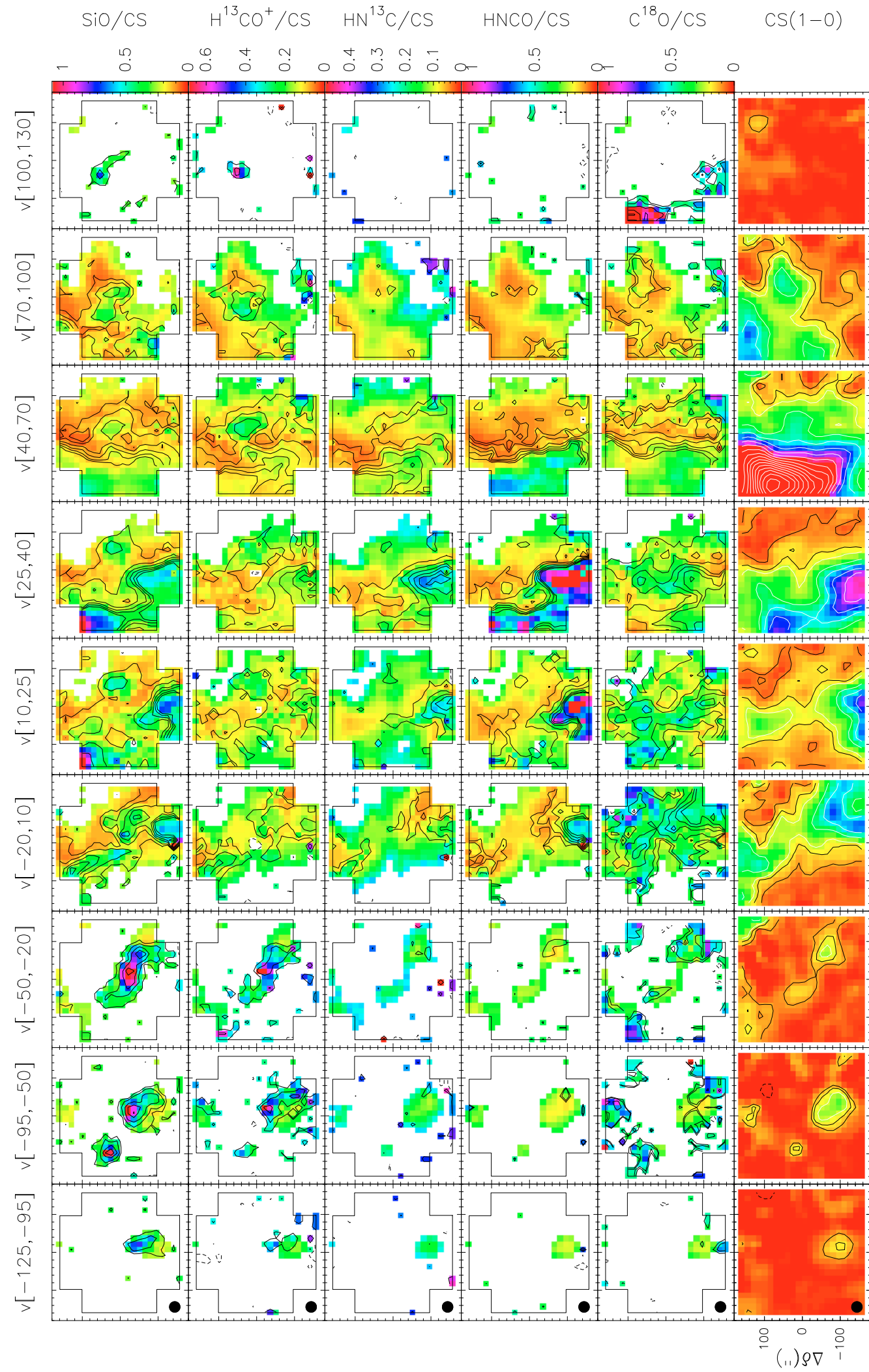

Fig. B.1. $\mathrm{X} / \mathrm{CS}(1-0)$ intensity ratios in the same selected velocity ranges as Fig. 2 . $\mathrm{X}$ stands for $\mathrm{SiO}(2-1), \mathrm{H}^{13} \mathrm{CO}^{+}(1-0)$, $\mathrm{HN}{ }^{13} \mathrm{C}(1-0)$, $\mathrm{HNCO}(5-4)$, and $\mathrm{C}^{18} \mathrm{O}(1-0)$. Molecular emission is shown in contour levels, which are $-3 \sigma$ (dashed contour), $3 \sigma$, from 2.0 to 8 in steps of $1.5 \mathrm{~K} \mathrm{~km} \mathrm{~s}^{-1}$. The $3 \sigma$ level of each molecule is the same as in Fig. 2. Intensity ratio maps take upper and lower limits into account, using the $3 \sigma$ value of each map as the limit value. The wedges to the left show the color scale of the different intensity ratios. Row at the bottom shows CS(1-0) maps of Tsuboi et al. (1999) to compare and show which pixels present meaningful ratios or are only limits (their contour levels and intensity scale are the same as in Fig. 2). 